TRANSACTIONS OF THE

AMERICAN MATHEMATICAL SOCIETY

Volume 364, Number 2, February 2012, Pages 1029-1066

S 0002-9947(2011)05484-X

Article electronically published on October 5, 2011

\title{
POSITIVE OPERATORS AND HAUSDORFF DIMENSION OF INVARIANT SETS
}

\author{
ROGER D. NUSSBAUM, AMIT PRIYADARSHI, AND SJOERD VERDUYN LUNEL
}

\begin{abstract}
In this paper we obtain theorems which give the Hausdorff dimension of the invariant set for a finite family of contraction mappings which are "infinitesimal similitudes" on a complete, perfect metric space. Our work generalizes the graph-directed construction of Mauldin and Williams (1988) and is related in its general setting to results of Schief (1996), but differs crucially in that the mappings need not be similitudes. We use the theory of positive linear operators and generalizations of the Krein-Rutman theorem to characterize the Hausdorff dimension as the unique value of $\sigma>0$ for which $r\left(L_{\sigma}\right)=1$, where $L_{\sigma}, \sigma>0$, is a naturally associated family of positive linear operators and $r\left(L_{\sigma}\right)$ denotes the spectral radius of $L_{\sigma}$. We also indicate how these results can be generalized to countable families of infinitesimal similitudes. The intent here is foundational: to derive a basic formula in its proper generality and to emphasize the utility of the theory of positive linear operators in this setting. Later work will explore the usefulness of the basic theorem and its functional analytic setting in studying questions about Hausdorff dimension.
\end{abstract}

\section{INTRODUCTION}

Given $N$ contraction mappings $\theta_{i}, 1 \leq i \leq N$, on a complete metric space $(X, d)$, there exists a unique, nonempty compact set $C$ such that $C=\bigcup_{i=1}^{N} \theta_{i}(C)$. $C$ is called an invariant set or an attractor for the family $\left\{\theta_{i}\right\}_{i=1}^{N}$. A general problem is to obtain theorems which allow the accurate estimation of the Hausdorff dimension of $C$. A well-studied case occurs when the maps $\theta_{i}$ are "similitudes", i.e., when for $1 \leq i \leq N$, there exists $r_{i}, 0<r_{i}<1$, with $d\left(\theta_{i}(x), \theta_{i}(y)\right)=r_{i} d(x, y)$ for all $x, y \in X$. If, in addition, $X$ is a normed linear space with metric $d$ derived from the norm on $X$ and if the similitudes $\theta_{i}$ are onto maps (which is necessarily true if $X$ is finite dimensional), then a theorem of Mazur and Ulam (see [14] or 23]) implies that each $\theta_{i}$ is an affine linear map. Moran [15] and Hutchinson [7] have studied the case that each $\theta_{i}, 1 \leq i \leq N$, is an affine linear similitude on a finite dimensional normed linear space $X$. Provided the "pieces" $\theta_{i}(C)$ do not overlap too much, they have proved that the Hausdorff dimension of $C$ is the same as the "similarity dimension" $\alpha$ and is determined by the equation $\sum_{i=1}^{N} r_{i}^{\alpha}=1$. More precisely, one needs the "open set condition", i.e., the assumption that there exists a nonempty, open set $U$ such that the sets $\theta_{i}(U)$ are contained in $U$ and are pairwise

Received by the editors February 1, 2010 and, in revised form, October 5, 2010.

2010 Mathematics Subject Classification. Primary 37F35, 28A80; Secondary 37C30, 47B65.

Key words and phrases. Hausdorff dimension, iterated function systems, positive operators, spectral radius.

The first author was supported in part by NSF Grant DMS-0701171.

(C)2011 American Mathematical Society 
disjoint. Mauldin and Williams [13] have generalized the idea of "self-similarity" to "graph self-similarity" which allows a larger class of sets like $C$, but still the maps in question are affine linear similitudes. Schief [22] works in a setting similar to ours and considers similitudes on general complete metric spaces and obtains analogous results. Interestingly, he has shown that in this generality, the open set condition is no longer sufficient and must be strengthened to the "strong open set condition".

It is of considerable interest (see [2, 3]) to allow maps $\theta_{i}$ which may not be affine linear. For example, in studying subsets of $\mathbb{R}$ defined by properties of their continued fraction expansions, one is led to maps $\theta_{i}:[0,1] \rightarrow[0,1]$ defined by $\theta_{i}(x)=\left(x+m_{i}\right)^{-1}, m_{i}$ a positive integer.

In this paper we shall consider a complete, perfect metric space $(X, d)$ and maps $\theta_{i}: X \rightarrow X, 1 \leq i \leq N$, where $\theta_{i}$ is a contraction mapping and an "infinitesimal similitude" (instead of a similitude) for $1 \leq i \leq N$. If $G$ is a bounded open subset of $\mathbb{C}, \theta_{j}: G \rightarrow G$ is analytic and $\overline{\theta_{j}(G)} \subset G$ for $1 \leq j \leq N$ and $X=\bigcup_{j=1}^{N} \overline{\theta_{j}(G)}$ with appropriate metric $d$, we obtain an important example for which the mappings $\theta_{j}: X \rightarrow X$ are contractions and infinitesimal similitudes. For the general definition of infinitesimal similitudes and their properties, see the beginning of $\S 3$. If $C$ denotes the nonempty, compact invariant set for $\left\{\theta_{i}\right\}_{i=1}^{N}$ and if $\theta_{i}(C)$ and $\theta_{j}(C)$ are disjoint for $1 \leq i<j \leq N$, we shall obtain below a formula for the Hausdorff dimension of $C$. In fact, we shall obtain such a formula in a setting similar to that of Mauldin and Williams, but using contractions and infinitesimal similitudes, rather than affine linear contractions which are similitudes.

The classical Krein-Rutman theorem (see [8) considers a positive (in the sense of mapping a suitable cone to itself), compact, linear map $T: X \rightarrow X$ which has positive spectral radius $r$ and asserts the existence of a positive eigenvector $v$ with $T(v)=r v$. Generalizations, particularly allowing noncompact $T$, can be found in [1, 9, 18, 19, 21. Our approach in this paper will be to use generalizations of the Krein-Rutman theorem. To each nonnegative real $\sigma$ we shall associate a positive linear operator $L_{\sigma}$ on a Banach space $Y$ of continuous functions. We shall prove that $L_{\sigma}$ has a positive eigenvector with eigenvalue $r\left(L_{\sigma}\right)$, the spectral radius of $L_{\sigma}$. We shall prove that $\sigma_{0}$, the desired Hausdorff dimension, is the unique value of $\sigma \geq 0$ for which $r\left(L_{\sigma}\right)=1$. We shall not use the "thermodynamic formalism". Curiously, we have found no references to the Krein-Rutman theorem in the Hausdorff dimension literature, despite its relevance. Analogues of the operator $L_{\sigma}$ we consider are sometimes called "Perron-Frobenius operators" or "Frobenius-Ruelle operators", although the theory originally developed by Perron and Frobenius is restricted to matrices with nonnegative entries, and generalizations to infinite dimensions pose substantial difficulties.

For the convenience of the reader we shall now state our main theorem in the simpler setting of iterated function systems on a compact, perfect metric space. For the more general case, see $\S 3$.

Let $(S, d)$ be a compact, perfect metric space. If $\theta: S \rightarrow S$, we shall say that $\theta$ is an infinitesimal similitude at $t \in S$ if for any sequences $\left(s_{k}\right)_{k}$ and $\left(t_{k}\right)_{k}$ with $s_{k} \neq t_{k}$ for $k \geq 1$ and $s_{k} \rightarrow t, t_{k} \rightarrow t$, the limit

$$
\lim _{k \rightarrow \infty} \frac{d\left(\theta\left(s_{k}\right), \theta\left(t_{k}\right)\right)}{d\left(s_{k}, t_{k}\right)}=:(D \theta)(t)
$$

exists and is independent of the particular sequences $\left(s_{k}\right)_{k}$ and $\left(t_{k}\right)_{k}$. We shall say that $\theta$ is an infinitesimal similitude on $S$ if $\theta$ is an infinitesimal similitude at $t$ for all $t \in S$. 
Assume that for $1 \leq i \leq N, \theta_{i}: S \rightarrow S$ is a Lipschitz map with Lipschitz constant $c_{i} \leq c<1$. Then we know that there exists a unique, compact, nonempty set $C \subset S$ with

$$
C=\bigcup_{i=1}^{N} \theta_{i}(C) .
$$

Assume the map $\theta_{i}: S \rightarrow S$ is an infinitesimal similitude on $S$ and the map $t \mapsto\left(D \theta_{i}\right)(t)$ is a strictly positive Hölder continuous function on $S$ for $1 \leq i \leq N$. For $\sigma \geq 0$, define $L_{\sigma}: C(S) \rightarrow C(S)$ by

$$
\left(L_{\sigma} f\right)(t)=\sum_{i=1}^{N}\left(\left(D \theta_{i}\right)(t)\right)^{\sigma} f\left(\theta_{i}(t)\right) .
$$

It follows (see Theorem 5.4 in 19]) that $L_{\sigma}$ has a strictly positive eigenvector $u_{\sigma}$ with eigenvalue equal to the spectral radius $r\left(L_{\sigma}\right)$ of $L_{\sigma}$. We also have the following lemma.

Lemma 1.1. The map $\sigma \mapsto r\left(L_{\sigma}\right)$ is continuous and strictly decreasing. Furthermore, there is a unique $\sigma_{0} \geq 0$ such that $r\left(L_{\sigma_{0}}\right)=1$.

We are now ready to state the theorem about the Hausdorff dimension of the invariant set $C$.

Theorem 1.2. Let $\theta_{i}: S \rightarrow S$ for $1 \leq i \leq N$ be infinitesimal similitudes and assume that the map $t \mapsto\left(D \theta_{i}\right)(t)$ is a strictly positive Hölder continuous function on $S$. Assume that $\theta_{i}: S \rightarrow S$ is a Lipschitz map with Lipschitz constant $c_{i} \leq c<1$ and let $C$ denote the unique invariant set such that

$$
C=\bigcup_{i=1}^{N} \theta_{i}(C) .
$$

Further, assume that $\theta_{i}, 1 \leq i \leq N$, satisfy

$$
\theta_{i}(C) \cap \theta_{j}(C)=\emptyset \text { for } 1 \leq i, j \leq N, i \neq j
$$

and are one-to-one on $C$. Then the Hausdorff dimension, $\operatorname{dim}(C)$ of $C$ is given by the unique $\sigma_{0}$ such that $r\left(L_{\sigma_{0}}\right)=1$.

To see that this is a special case of our general theory appearing in later sections, let $V=\{1\}, \mathcal{E}=\{1,2, \ldots, N\}$ and $\Gamma=V \times \mathcal{E}$ in the terminology below.

We should remark that our proofs require that the pieces $\theta_{i}(C), 1 \leq i \leq N$, be pairwise disjoint. It would be very interesting to find variant arguments which allowed some overlap. For instance, we do not know if the strong open set condition is sufficient to get the results in this generality.

The paper is organised as follows. In $\S 2$ we introduce the basic setup and prove the existence of the invariant set. In $\S 3$ we discuss the Perron-Frobenius operators. In $\S 4$ we give the main theorems about the Hausdorff dimension of the invariant set. In $\S 5$ the results are extended to infinite iterated function systems, and we show that the class of complex continued fractions previously studied by Mauldin and Urbański [12] is also covered by our theorems. In $\S 6$ we show that choosing an appropriate metric gives a large class of examples of iterated function systems to which our theory can be applied. For instance, we discuss the CarathéodoryReiffen-Finsler $(\mathrm{CRF})$ metric on bounded open subsets of $\mathbb{C}$. 


\section{INVARIANT SETS}

Let $V$ and $\mathcal{E}$ be finite sets and for each $v \in V$, let $\left(S_{v}, d_{v}\right)$ be a complete metric space. Let $\Gamma$ be a subset of $V \times \mathcal{E}$, and $\alpha: \Gamma \rightarrow V$. For each $(v, e) \in \Gamma$, let $\theta_{(v, e)}: S_{v} \rightarrow S_{\alpha(v, e)}$ be a Lipschitz map with $\operatorname{Lip}\left(\theta_{(v, e)}\right) \leq c<1$. Recall that a map $\psi:\left(S_{1}, d_{1}\right) \rightarrow\left(S_{2}, d_{2}\right)$ is said to be Lipschitz if there is a constant $c$ such that $d_{2}(\psi(s), \psi(t)) \leq c d_{1}(s, t) \forall s, t \in S_{1}$, and

$$
\operatorname{Lip}(\psi):=\sup \left\{\frac{d_{2}(\psi(s), \psi(t))}{d_{1}(s, t)}: s, t \in S_{1}, s \neq t\right\} .
$$

The notation and assumptions will be as in the preceding paragraph for the remainder of the paper. We shall keep in mind two important particular cases.

Example 2.1 (The Mauldin-Williams graph). Let $V$ be the set of vertices and $\mathcal{E}$ be the set of edges of a directed multigraph. Let $i(e)$ and $t(e)$ denote the initial and terminal vertices of edge $e \in \mathcal{E}$. The set $\Gamma$ is defined by $(v, e) \in \Gamma$ if and only if $v=t(e)$. The map $\alpha$ in this case is $\alpha(v, e)=i(e)$. See chapter 4.3 in [4] for a discussion of the Mauldin-Williams graph.

Example 2.2. Let $(T, d)$ be a bounded complete metric space. Assume $T=$ $\bigcup_{k=1}^{p} T_{k}$, where each $T_{k}$ is a closed subset of $T$ and $T_{k} \cap T_{l}=\emptyset$ for $k \neq l$. For $1 \leq i \leq m$, let $\theta_{i}: T \rightarrow T$ be a continuous map such that $\theta_{i}\left(T_{k}\right) \subset T_{\nu(i, k)}$, $1 \leq k \leq p$, where $\nu(i, k) \in\{1,2, \ldots, p\}$, and $\operatorname{Lip}\left(\left.\theta_{i}\right|_{T_{k}}\right) \leq c<1$. In this case, we take $V=\{k: 1 \leq k \leq p\}, S_{k}=T_{k}$ for $1 \leq k \leq p, \mathcal{E}=\{i: 1 \leq i \leq m\}, \Gamma=V \times \mathcal{E}$. The map $\alpha(k, i)=\nu(i, k), 1 \leq k \leq p, 1 \leq i \leq m$, and $\theta_{(k, i)}=\left.\theta_{i}\right|_{T_{k}}$.

For $u \in V$, define

$$
\Gamma_{u}=\{(v, e) \in \Gamma: \alpha(v, e)=u\}
$$

and

For $n \geq 1$, define

$$
E_{u}=\{e \in \mathcal{E}:(u, e) \in \Gamma\}
$$

$$
\Gamma^{(n)}=\left\{\left[\left(v_{1}, e_{1}\right), \ldots,\left(v_{n}, e_{n}\right)\right]:\left(v_{i}, e_{i}\right) \in \Gamma, \alpha\left(v_{i+1}, e_{i+1}\right)=v_{i}, 1 \leq i \leq n-1\right\} .
$$

For $u \in V, n \geq 1$, define

$$
\Gamma_{u}^{(n)}=\left\{\left[\left(v_{1}, e_{1}\right), \ldots,\left(v_{n}, e_{n}\right)\right] \in \Gamma^{(n)}: \alpha\left(v_{1}, e_{1}\right)=u\right\} .
$$

Define $V_{\infty}=\left\{u \in V: \Gamma_{u}^{(n)} \neq \emptyset \quad \forall n \geq 1\right\}$.

Before we prove the next theorem we need to recall the definition of the Hausdorff metric. Let $(S, d)$ be a complete metric space. If $A \subset S$, we define the diameter of $A$ by

$$
\operatorname{diam}(A)=\sup \{d(s, t): s, t \in A\} .
$$

We shall say that $A$ is bounded if $\operatorname{diam}(A)<\infty$. For $A \subset S$ and $s \in S$ we define

$$
d(s, A)=\inf \{d(s, a): a \in A\} .
$$

If $A \subset S$ and $\delta>0$, we define $N_{\delta}(A)$ by

$$
N_{\delta}(A)=\{s \in S: d(s, A)<\delta\} .
$$

If $A$ and $B$ are nonempty, closed, bounded subsets of $S$, we define

$$
D(A, B)=\inf \left\{\delta>0: A \subset N_{\delta}(B) \text { and } B \subset N_{\delta}(A)\right\} .
$$

If $\mathcal{B}(S)$ denotes the collection of nonempty, closed, bounded subsets of $S$, then it follows that $(\mathcal{B}(S), D)$ is a metric space. The metric $D$ is called the Hausdorff 
metric. It is known (see [16, Exercise 7, pages 280-281) that if $(S, d)$ is a complete metric space, then $(\mathcal{B}(S), D)$ is also a complete metric space.

Theorem 2.3. Let $V, \mathcal{E}, \Gamma, \alpha$ be given as before, and $\theta_{(v, e)}: S_{v} \rightarrow S_{\alpha(v, e)}$ be a Lipschitz map with $\operatorname{Lip}\left(\theta_{(v, e)}\right) \leq c<1$ for all $(v, e) \in \Gamma$. Assume $\Gamma_{u} \neq \emptyset$ for all $u \in V$. Then there exists a unique list $\left(C_{v}\right)_{v \in V}$ of nonempty closed bounded sets $C_{v} \subset S_{v}$ such that

$$
C_{u}=\overline{\bigcup_{(v, e) \in \Gamma_{u}} \theta_{(v, e)}\left(C_{v}\right)}
$$

for all $u \in V$. Furthermore, $C_{v}$ is indeed compact for all $v \in V$, so we may remove the closure in the above equation.

Proof. Let $\mathcal{B}\left(S_{v}\right)$ denote the collection of closed, bounded nonempty subsets of $S_{v}$ with the Hausdorff metric $D_{v}$. Then since $S_{v}$ is a complete metric space, we know that $\mathcal{B}\left(S_{v}\right)$ is a complete metric space. So the finite Cartesian product $\prod_{v \in V} \mathcal{B}\left(S_{v}\right)$ with the sup metric is also a complete metric space. Define the map $\Theta: \prod_{v \in V} \mathcal{B}\left(S_{v}\right) \rightarrow \prod_{v \in V} \mathcal{B}\left(S_{v}\right)$ by

$$
\Theta\left(\left(A_{v}\right)_{v \in V}\right)=\left(\overline{\bigcup_{(v, e) \in \Gamma_{u}} \theta_{(v, e)}\left(A_{v}\right)}\right)_{u \in V} .
$$

Note that for any $u \in V, \overline{\bigcup_{(v, e) \in \Gamma_{u}} \theta_{(v, e)}\left(A_{v}\right)}$ is nonempty because $\Gamma_{u}$ is nonempty by assumption, and $A_{v}$ is nonempty for each $v \in V$. Also it is bounded because $\theta_{(v, e)}\left(A_{v}\right)$, being the image of bounded set $A_{v}$ under a Lipschitz map $\theta_{(v, e)}$, is bounded, and a finite union of bounded sets is bounded. Thus the map $\Theta$ is well defined.

We claim that $\Theta$ is a contraction map. Let $A=\left(A_{v}\right)_{v \in V}$ and $B=\left(B_{v}\right)_{v \in V}$ be in $\prod_{v \in V} \mathcal{B}\left(S_{v}\right)$. Then $D(A, B)=\max _{v \in V} D_{v}\left(A_{v}, B_{v}\right)$ and

$$
D(\Theta(A), \Theta(B))=\max _{u \in V} D_{u}\left(\overline{\bigcup_{(v, e) \in \Gamma_{u}} \theta_{(v, e)}\left(A_{v}\right)}, \bigcup_{(v, e) \in \Gamma_{u}} \theta_{(v, e)}\left(B_{v}\right)\right) .
$$

Let $\delta>D(A, B)$, and take any $(v, e) \in \Gamma_{u}, a_{v} \in A_{v}$. Since $D_{v}\left(A_{v}, B_{v}\right) \leq$ $D(A, B)<\delta$, there exists $b_{v} \in B_{v}$ such that $d_{v}\left(a_{v}, b_{v}\right)<\delta$. Then

$$
d_{u}\left(\theta_{(v, e)}\left(a_{v}\right), \theta_{(v, e)}\left(b_{v}\right)\right) \leq c d_{v}\left(a_{v}, b_{v}\right)<c \delta .
$$

This shows that $\bigcup_{(v, e) \in \Gamma_{u}} \theta_{(v, e)}\left(A_{v}\right)$ is contained in a $c \delta$-neighborhood of

$$
\bigcup_{(v, e) \in \Gamma_{u}} \theta_{(v, e)}\left(B_{v}\right)
$$

Similarly we can prove the other way. So,

$$
D_{u}\left(\overline{\bigcup_{(v, e) \in \Gamma_{u}} \theta_{(v, e)}\left(A_{v}\right)}, \overline{\bigcup_{(v, e) \in \Gamma_{u}} \theta_{(v, e)}\left(B_{v}\right)}\right) \leq c \delta
$$


for all $u \in V$. Hence, $D(\Theta(A), \Theta(B)) \leq c \delta$. Since $\delta>D(A, B)$ was arbitrary, we have proved that $D(\Theta(A), \Theta(B)) \leq c D(A, B)$.

Therefore we have a contraction map $\Theta$ on a complete metric space. By the contraction mapping theorem, $\Theta$ has a unique fixed point, say $\left(C_{v}\right)_{v \in V}$. Thus we have $C_{u}=\overline{\bigcup_{(v, e) \in \Gamma_{u}} \theta_{(v, e)}\left(C_{v}\right)}$.

To see that $C_{v}$ is compact for all $v \in V$, let us restrict the map $\Theta$ to $\prod_{v \in V} \mathcal{K}\left(S_{v}\right)$, where $\mathcal{K}\left(S_{v}\right)$ denotes the collection of nonempty compact subsets of $S_{v}$ with the Hausdorff metric $D_{v}$. It is a straightforward exercise to prove that $\prod_{v \in V} \mathcal{K}\left(S_{v}\right)$ is a closed subset of the complete metric space $\prod_{v \in V} \mathcal{B}\left(S_{v}\right)$, so $\prod_{v \in V} \mathcal{K}\left(S_{v}\right)$ is itself a complete metric space. Then $\Theta$ maps the complete metric space $\prod_{v \in V} \mathcal{K}\left(S_{v}\right)$ into itself, and is a contraction map as seen above. Thus $\Theta$ has a unique fixed point in $\prod_{v \in V} \mathcal{K}\left(S_{v}\right)$. The fixed point must be the same as $\left(C_{v}\right)_{v \in V}$, since otherwise the original map $\Theta$ would have two fixed points. Therefore $C_{v}$ is compact for all $v \in V$, and the theorem is proved.

Remark 2.4. The assumption $\Gamma_{u} \neq \emptyset$ for all $u \in V$ in the above theorem may be too strong for some examples. A weaker assumption under which we can prove the existence of an invariant list is $V_{\infty} \neq \emptyset$. Note that $\Gamma_{u} \neq \emptyset$ for all $u \in V$ implies $V_{\infty}=V$, so $V_{\infty} \neq \emptyset$.

First we claim that $u \in V_{\infty}$ implies that there exists $v \in V_{\infty}$ with $(v, e) \in \Gamma_{u}$ for some $e \in \mathcal{E}$. Suppose not. Then for all $(v, e) \in \Gamma_{u}, v \notin V_{\infty}$. This implies, since $V$ is a finite set, that there exists $n \geq 1$ such that $\Gamma_{v}^{(n)}=\emptyset$ for all $(v, e) \in \Gamma_{u}$. But since $u \in V_{\infty}$, there exists $\left[\left(v_{1}, e_{1}\right),\left(v_{2}, e_{2}\right), \ldots,\left(v_{n+1}, e_{n+1}\right)\right] \in \Gamma_{u}^{(n+1)}$, which implies $\left[\left(v_{2}, e_{2}\right), \ldots,\left(v_{n+1}, e_{n+1}\right)\right] \in \Gamma_{v_{1}}^{(n)}$ and since $\alpha\left(v_{1}, e_{1}\right)=u,\left(v_{1}, e_{1}\right) \in \Gamma_{u}$. This contradicts $\Gamma_{v}^{(n)}=\emptyset$ for all $(v, e) \in \Gamma_{u}$, hence the claim.

Now consider the map $\Theta: \prod_{v \in V_{\infty}} \mathcal{B}\left(S_{v}\right) \rightarrow \prod_{v \in V_{\infty}} \mathcal{B}\left(S_{v}\right)$ by

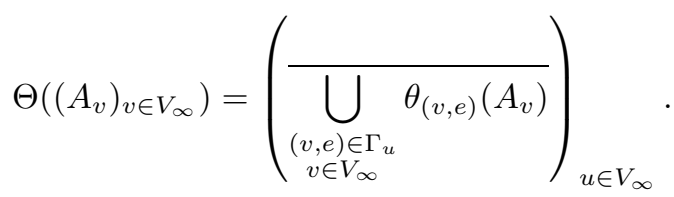

Note that $\Theta$ is well defined because of the above claim. Again by the contraction mapping theorem, we have $\left(C_{v}\right)_{v \in V_{\infty}}, C_{v} \subset S_{v}$ is compact such that

$$
C_{u}=\bigcup_{\substack{(v, e) \in \Gamma_{u} \\ v \in V_{\infty}}} \theta_{(v, e)}\left(C_{v}\right) .
$$

Equivalently, under the assumption that $V_{\infty} \neq \emptyset$, all we have done is replaced $V$ by $\hat{V}:=V_{\infty}, \Gamma$ by $\hat{\Gamma}:=\{(v, e) \in \Gamma \mid v \in \hat{V}\}$ and $\alpha$ by $\hat{\alpha}:=\alpha \mid \hat{\Gamma}$, and then applied Theorem 2.3 ,

Remark 2.5. Applying the previous result to Example 2.2, we get nonempty compact sets $C_{k} \subset T_{k}$ for $k \in V_{\infty}$ such that

$$
C_{k}=\bigcup_{\substack{\nu(i, l)=k \\ l \in V_{\infty}}} \theta_{i}\left(C_{l}\right) \text { for } k \in V_{\infty} .
$$


If we let $C=\bigcup_{l \in V_{\infty}} C_{l}$, then $C$ is a nonempty compact set and it satisfies

$$
C=\bigcup_{i=1}^{m} \theta_{i}(C) .
$$

Thus we have a compact invariant set for the family of maps $\theta_{i}, 1 \leq i \leq m$.

Remark 2.6. We can relax the condition $\operatorname{Lip}\left(\theta_{(v, e)}\right) \leq c<1$ for all $(v, e) \in \Gamma$ in the previous theorem to the following weaker condition. Suppose that for some fixed $n \geq 1$, the composition of any $n$ of the maps $\theta_{(v, e)}$, whenever the composition is defined, is Lipschitz with Lipschitz constant $\leq c<1$. Then it is easy to see that $\Theta^{n}$ is a contraction map on a complete metric space, where $\Theta$ is the map defined in the proof of the theorem. It is then well known that the map $\Theta$ has a unique fixed point. Thus the conclusion of the previous theorem holds under this weaker assumption.

\section{Perron-Frobenius operators}

From now on, let $V=\{1,2, \ldots, p\}$ with $S_{1}, S_{2}, \ldots, S_{p}$ the corresponding complete metric spaces. We do not necessarily assume that $S_{j}, 1 \leq j \leq p$, is compact. Let

$$
X_{i}=C_{b}\left(S_{i}\right)=\left\{f: S_{i} \rightarrow \mathbb{R}: f \text { is continuous and bounded }\right\}
$$

for $1 \leq i \leq p$ with $\|f\|=\sup _{s \in S_{i}}|f(s)|$.

Define a linear map $A: X_{1} \times X_{2} \times \cdots \times X_{p} \rightarrow X_{1} \times X_{2} \times \cdots \times X_{p}$ by

$$
(A f)_{j}(s)=\sum_{e \in E_{j}} b_{(j, e)}(s) f_{\alpha(j, e)}\left(\theta_{(j, e)}(s)\right) \quad \text { for } s \in S_{j},
$$

where $f=\left(f_{1}, f_{2}, \ldots, f_{p}\right)$ and the functions $b_{(j, e)} \in X_{j}$ are given. We assume throughout this section that $E_{j}=\{e \in \mathcal{E}:(j, e) \in \Gamma\}$ is nonempty for all $j \in V$.

Define for $M>0, \lambda \geq 0,1 \leq j \leq p$,

$$
K_{j}(M, \lambda)=\left\{f \in X_{j}: 0 \leq f(s) \leq f(t) \exp \left(M\left(d_{j}(s, t)\right)^{\lambda}\right) \text { for all } s, t \in S_{j}\right\} .
$$

Remark 3.1. From the definition, it follows that if $f \in K_{j}(M, \lambda)$ and $f(t)=0$ for some $t \in S_{j}$, then $f(s)=0$ for all $s \in S_{j}$. Thus $f \in K_{j}(M, \lambda)$ implies that either $f$ is identically zero on $S_{j}$ or $f$ is strictly positive on $S_{j}$.

If $Y$ is a real Banach space, a closed set $K \subset Y$ is called a closed cone if $\lambda K+\mu K \subset K$ for all $\lambda \geq 0, \mu \geq 0$ and $K \cap(-K)=\{0\}$.

The following lemma follows by the same argument used in Lemma 5.4, p. 89, in [19]. We give the proof for the reader's convenience.

Lemma 3.2. Let $K_{j}:=K_{j}(M, \lambda)$ be as defined by (3.2) with $\lambda>0$. Then $K_{j}$ is a closed cone in $\left(X_{j},\|\|.\right)$, and $\left\{f \in K_{j}:\|f\| \leq 1\right\}$ is equicontinuous.

Proof. It is easy to verify that $K_{j}$ is a closed cone, and the proof is left to the reader. To prove the equicontinuity of $\left\{f \in K_{j}:\|f\| \leq 1\right\}$ let $f \in K_{j}$ with $\|f\| \leq 1$. We claim that for any $s, t \in S_{j}$ we have

$$
|f(s)-f(t)| \leq M\left(d_{j}(s, t)\right)^{\lambda} .
$$

According to the previous remark, either $f$ is identically zero on $S_{j}$ or $f$ is strictly positive on $S_{j}$. The inequality is obvious in the first case. In the latter case, we may assume that $0<f(s) \leq f(t) \leq 1$. The definition of $K_{j}$ implies that

$$
|\ln (f(s))-\ln (f(t))| \leq M\left(d_{j}(s, t)\right)^{\lambda} .
$$


The mean value theorem implies that for some $\xi$ with $\ln (f(s)) \leq \xi \leq \ln (f(t)) \leq 0$ we have

$$
\begin{aligned}
|f(s)-f(t)| & =\exp (\ln (f(t)))-\exp (\ln (f(s))) \\
& =\exp (\xi)|\ln (f(t))-\ln (f(s))| \leq M\left(d_{j}(s, t)\right)^{\lambda} .
\end{aligned}
$$

Since this is true for any $f \in K_{j}$ with $\|f\| \leq 1$, equicontinuity follows.

Lemma 3.3. Assume for some $M_{0}>0$ and $\lambda>0$ that $b_{(j, e)} \in K_{j}\left(M_{0}, \lambda\right)$ for all $(j, e) \in \Gamma$. Then there exists $M>0$ so that the map $A$ defined above maps $\prod_{i=1}^{p} K_{i}(M, \lambda)$ into itself.

Proof. Let $f_{i} \in K_{i}(M, \lambda)$ for $1 \leq i \leq p$ and $s, t \in S_{j}$. Then

$$
(A f)_{j}(s)=\sum_{e \in E_{j}} b_{(j, e)}(s) f_{\alpha(j, e)}\left(\theta_{(j, e)}(s)\right) .
$$

Since $b_{(j, e)} \in K_{j}\left(M_{0}, \lambda\right), b_{(j, e)}(s) \leq b_{(j, e)}(t) \exp \left(M_{0}\left(d_{j}(s, t)\right)^{\lambda}\right)$. Also

$$
\begin{aligned}
f_{\alpha(j, e)}\left(\theta_{(j, e)}(s)\right) & \leq f_{\alpha(j, e)}\left(\theta_{(j, e)}(t)\right) \exp \left(M\left(d_{\alpha(j, e)}\left(\theta_{(j, e)}(s), \theta_{(j, e)}(t)\right)\right)^{\lambda}\right) \\
& \leq f_{\alpha(j, e)}\left(\theta_{(j, e)}(t)\right) \exp \left(M c^{\lambda}\left(d_{j}(s, t)\right)^{\lambda}\right) .
\end{aligned}
$$

Thus

$$
\begin{aligned}
(A f)_{j}(s) & =\sum_{e \in E_{j}} b_{(j, e)}(s) f_{\alpha(j, e)}\left(\theta_{(j, e)}(s)\right) \\
& \leq \sum_{e \in E_{j}} b_{(j, e)}(t) f_{\alpha(j, e)}\left(\theta_{(j, e)}(t)\right) \exp \left(\left(M_{0}+M c^{\lambda}\right)\left(d_{j}(s, t)\right)^{\lambda}\right) \\
& =(A f)_{j}(t) \exp \left(\left(M_{0}+M c^{\lambda}\right)\left(d_{j}(s, t)\right)^{\lambda}\right) .
\end{aligned}
$$

So, if we choose $M$ such that $M_{0}+M c^{\lambda} \leq M$, which can be done because $c<1$, then

$$
(A f)_{j}(s) \leq(A f)_{j}(t) \exp \left(M\left(d_{j}(s, t)\right)^{\lambda}\right)
$$

so $(A f)_{j} \in K_{j}(M, \lambda)$ for $1 \leq j \leq p$.

We should note that observations similar to Lemma 3.3 have been made earlier by other authors. See the proof of Theorem 5.4 in [19] and [2], for example.

We shall use the following notation:

$$
\begin{gathered}
\bar{\Gamma}^{(n)}:=\left\{\left[\left(j_{1}, e_{1}\right), \ldots,\left(j_{n}, e_{n}\right)\right]:\left(j_{i}, e_{i}\right) \in \Gamma, 1 \leq i \leq n, \alpha\left(j_{i}, e_{i}\right)=j_{i+1}, 1 \leq i<n\right\}, \\
\bar{\Gamma}_{j}^{(n)}:=\left\{\left[\left(j_{1}, e_{1}\right), \ldots,\left(j_{n}, e_{n}\right)\right] \in \bar{\Gamma}^{(n)}: j_{1}=j\right\} .
\end{gathered}
$$

We shall also use $(J, E)$, where $J=\left(j_{1}, \ldots, j_{n}\right), E=\left(e_{1}, \ldots, e_{n}\right)$ as a shorthand notation for $\left[\left(j_{1}, e_{1}\right), \ldots,\left(j_{n}, e_{n}\right)\right] \in \bar{\Gamma}^{(n)}$.

For $(J, E)=\left[\left(j_{1}, e_{1}\right), \ldots,\left(j_{n}, e_{n}\right)\right] \in \bar{\Gamma}^{(n)}$, define for $s \in S_{j_{1}}$,

$$
b_{(J, E)}(s):=b_{\left(j_{1}, e_{1}\right)}(s) b_{\left(j_{2}, e_{2}\right)}\left(\theta_{\left(j_{1}, e_{1}\right)}(s)\right) \cdots b_{\left(j_{n}, e_{n}\right)}\left(\theta_{\left(j_{n-1}, e_{n-1}\right)} \circ \cdots \circ \theta_{\left(j_{1}, e_{1}\right)}(s)\right)
$$

and

$$
\theta_{(J, E)}(s):=\theta_{\left(j_{n}, e_{n}\right)} \circ \cdots \circ \theta_{\left(j_{1}, e_{1}\right)}(s)
$$


Let us compute $A^{2}$ :

$$
\begin{aligned}
\left(A^{2} f\right)_{j_{1}}(s) & =(A(A f))_{j_{1}}(s) \\
& =\sum_{e_{1}:\left(j_{1}, e_{1}\right) \in \Gamma} b_{\left(j_{1}, e_{1}\right)}(s)(A f)_{\alpha\left(j_{1}, e_{1}\right)}\left(\theta_{\left(j_{1}, e_{1}\right)}(s)\right) .
\end{aligned}
$$

Using

$$
(A f)_{\alpha\left(j_{1}, e_{1}\right)}\left(\theta_{\left(j_{1}, e_{1}\right)}(s)\right)=\sum_{\substack{e_{2}:\left(j_{2}, e_{2}\right) \in \Gamma \\ j_{2}=\alpha\left(j_{1}, e_{1}\right)}} b_{\left(j_{2}, e_{2}\right)}\left(\theta_{\left(j_{1}, e_{1}\right)}(s)\right) f_{\alpha\left(j_{2}, e_{2}\right)}\left(\theta_{\left(j_{2}, e_{2}\right)}\left(\theta_{\left(j_{1}, e_{1}\right)}(s)\right)\right),
$$

we get

$$
\left(A^{2} f\right)_{j_{1}}(s)=\sum_{(J, E) \in \bar{\Gamma}_{j_{1}}^{(2)}} b_{(J, E)}(s) f_{\alpha\left(j_{2}, e_{2}\right)}\left(\theta_{(J, E)}(s)\right) .
$$

This computation suggests the formula for $A^{n}$ given in the following lemma.

Lemma 3.4. Let $n \geq 1$. Then for $f=\left(f_{1}, f_{2}, \ldots, f_{p}\right) \in \prod_{i=1}^{p} X_{i}$ and $1 \leq j_{1} \leq p$,

$$
\left(A^{n} f\right)_{j_{1}}(s)=\sum_{(J, E) \in \bar{\Gamma}_{j_{1}}^{(n)}} b_{(J, E)}(s) f_{\alpha\left(j_{n}, e_{n}\right)}\left(\theta_{(J, E)}(s)\right), \quad s \in S_{j_{1}} .
$$

Also the operator norm of $A^{n}$ is given by

$$
\left\|A^{n}\right\|=\max _{1 \leq j \leq p} \sup _{s \in S_{j}} \sum_{(J, E) \in \bar{\Gamma}_{j}^{(n)}} b_{(J, E)}(s) .
$$

Proof. The equation for $A^{n}$ follows by a simple induction on $n$. If $f \in \prod_{i=1}^{p} X_{i}$ with $\|f\| \leq 1$, i.e., $\left|f_{j}(s)\right| \leq 1 \forall s \in S_{j}, 1 \leq j \leq p$, then the equation for $\left(A^{n} f\right)_{j}(s)$ gives

$$
\left|\left(A^{n} f\right)_{j}(s)\right| \leq \sum_{(J, E) \in \bar{\Gamma}_{j}^{(n)}} b_{(J, E)}(s)
$$

Taking the supremum over $s \in S_{j}$ and then the maximum over $1 \leq j \leq p$ gives

$$
\left\|A^{n}\right\| \leq \max _{1 \leq j \leq p} \sup _{s \in S_{j}} \sum_{(J, E) \in \bar{\Gamma}_{j}^{(n)}} b_{(J, E)}(s) .
$$

If we take $f=\left(f_{1}, f_{2}, \ldots, f_{p}\right)$, where $f_{j}$ is identically equal to one on $S_{j}$, then

$$
\left(A^{n} f\right)_{j}(s)=\sum_{(J, E) \in \bar{\Gamma}_{j}^{(n)}} b_{(J, E)}(s) .
$$

Therefore we get the equation for $\left\|A^{n}\right\|$.

Lemma 3.5. Let $\left(S_{j}\right)_{j=1}^{p}$ be bounded complete metric spaces, assume that $\Gamma_{j}:=$ $\{(k, e) \in \Gamma: \alpha(k, e)=j\}$ is nonempty for $1 \leq j \leq p$, and let $\left(C_{j}\right)_{j=1}^{p}$ be the unique invariant list of compact sets given by Theorem 2.3 . Let $(J, E)=$ $\left[\left(j_{1}, e_{1}\right), \ldots,\left(j_{n}, e_{n}\right)\right] \in \bar{\Gamma}^{(n)}$ and $\theta_{(J, E)}(s)=\theta_{\left(j_{n}, e_{n}\right)} \circ \cdots \circ \theta_{\left(j_{1}, e_{1}\right)}(s), s \in S_{j_{1}}$. Then there exists $M_{1}>0$ such that for all $n \geq 1$,

$$
d_{\alpha\left(j_{n}, e_{n}\right)}\left(\theta_{(J, E)}(s), C_{\alpha\left(j_{n}, e_{n}\right)}\right) \leq M_{1} c^{n} \quad \forall s \in S_{j_{1}},
$$

where $c<1$ is the constant such that $\operatorname{Lip}\left(\theta_{(j, e)}\right) \leq c$ for all $(j, e) \in \Gamma$. 
Proof. Since the metric spaces $\left(S_{j}\right), 1 \leq j \leq p$ are bounded we can find $M_{1}$ so that $d_{j}\left(s, C_{j}\right) \leq M_{1} \forall s \in S_{j}, 1 \leq j \leq p$. Let $(j, e) \in \Gamma$ and $s \in S_{j}$. Then we can find $t \in C_{j}$ such that $d_{j}(s, t) \leq M_{1}$. Since $\theta_{(j, e)}(t) \in C_{\alpha(j, e)}$,

$$
d_{\alpha(j, e)}\left(\theta_{(j, e)}(s), C_{\alpha(j, e)}\right) \leq d_{\alpha(j, e)}\left(\theta_{(j, e)}(s), \theta_{(j, e)}(t)\right) \leq c d_{j}(s, t) \leq c M_{1} .
$$

The result now follows easily by an induction on $n$.

Let us recall the definition of Kuratowski's measure of noncompactness $\beta$. If $(S, d)$ is a metric space and $B \subset S$ is a bounded set, then $\beta(B)$ is defined by

$$
\beta(B)=\inf \left\{\delta>0: B=\bigcup_{j=1}^{k} B_{j}, k<\infty \text { and } \operatorname{diam}\left(B_{j}\right) \leq \delta \text { for } 1 \leq j \leq k\right\} .
$$

Suppose that $K$ is a closed cone in a Banach space $Y$ and $L: Y \rightarrow Y$ is a bounded linear map with $L(K) \subset K$. Define

$$
\|L\|_{K}=\sup \{\|L(y)\|: y \in K,\|y\| \leq 1\} .
$$

Define $r_{K}(L)$, the cone spectral radius of $L$, and $\sigma_{K}(L)$, by

$$
\begin{gathered}
{\left[r_{K}(L):=\lim _{n \rightarrow \infty}\left\|L^{n}\right\|_{K}^{1 / n}\right. \text { and }} \\
\sigma_{K}(L):=\limsup _{n \rightarrow \infty}\left(\beta\left(L^{n}(U)\right)\right)^{1 / n},
\end{gathered}
$$

where $U=\{y \in K:\|y\| \leq 1\}$.

It is a special case of results in [10] that if $\sigma_{K}(L)<r_{K}(L)$, then there exists $y \in K \backslash\{0\}$ with $L(y)=r y, r=r_{K}(L)$. (Note that the definition in [10] of $\rho_{K}(L)$, the cone essential spectral radius of $L$, satisfies $\rho_{K}(L) \leq \sigma_{K}(L)$. The definition of $\rho_{K}(L)$ in [10] differs from that in [9] and [18. It is shown in [10] that the earlier definition has some serious deficiencies.) We shall use this result to prove the existence of a nonzero eigenvector for the map $A$ given by (3.1). Alternatively, if all the sets $S_{j}$ were compact, an argument similar to the one used to prove Theorem 5.4 in [19] would give the following theorem.

Theorem 3.6. Consider the map $A$ defined on $\prod_{j=1}^{p} C_{b}\left(S_{j}\right)$ by

$$
(A f)_{j}(s)=\sum_{e \in E_{j}} b_{(j, e)}(s) f_{\alpha(j, e)}\left(\theta_{(j, e)}(s)\right) \quad \text { for } s \in S_{j}
$$

where $f=\left(f_{1}, f_{2}, \ldots, f_{p}\right)$. Assume that $S_{j}$ is a bounded, complete metric space for $1 \leq j \leq p$. Also assume that $\Gamma_{j} \neq \emptyset$ and $E_{j} \neq \emptyset$ for $1 \leq j \leq p$ and that for some $M_{0}>0$ and $\lambda>0, b_{(j, e)} \in K_{j}\left(M_{0}, \lambda\right)$ for all $(j, e) \in \Gamma$. Let $K$ be the cone $\prod_{j=1}^{p} K_{j}(M, \lambda)$, where $M_{0}+c^{\lambda} M \leq M$. Then $\left\|A^{n}\right\|_{K}=\left\|A^{n}\right\|$ for all $n \geq 1$, where $\left\|A^{n}\right\|$ is given by (3.4), and $r_{K}(A)=\lim _{n \rightarrow \infty}\left\|A^{n}\right\|^{\frac{1}{n}}$. If $r_{K}(A)>0$, there exists $u=\left(u_{1}, u_{2}, \ldots, u_{p}\right) \in K \backslash\{0\}$ with $A u=r u$, where $r=r_{K}(A)$. If $b_{(j, e)}(s)>0$ for all $(j, e) \in \Gamma$ and all $s \in S_{j}$, then $r_{K}(A)>0$.

Proof. It is enough to show that $\sigma_{K}(A)<r_{K}(A)$. Let $\left(C_{j}\right)_{j=1}^{p}$ be the unique invariant list of nonempty compact sets given by Theorem 2.3 and let $C=\prod_{j=1}^{p} C_{j}$. Let $\mathcal{U}=\{f \in K:\|f\| \leq 1\}$. From Lemma 3.2 we know that $\mathcal{U}$ is equicontinuous. Let us write $\left.\mathcal{U}\right|_{C}=\left\{\left.f\right|_{C}: f \in \mathcal{U}\right\}$. Then $\left.\mathcal{U}\right|_{C}$ is a bounded equicontinuous family of functions from the compact set $C$ into $\mathbb{R}^{p}$. So, by Ascoli's theorem, it is totally bounded. Therefore, given $\epsilon>0$, we can write $\mathcal{U}=\bigcup_{l=1}^{q} \mathcal{U}_{l}, q<\infty$, such that 
$\left\|\left.f\right|_{C}-\left.g\right|_{C}\right\| \leq \epsilon$ provided $f$ and $g$ are in the same $\mathcal{U}_{l}$. Let $f, g \in \mathcal{U}$ and $1 \leq j \leq p$. Then using Lemma 3.4 we have for $s \in S_{j}$,

$$
\begin{aligned}
& \left|\left(A^{n} f\right)_{j}(s)-\left(A^{n} g\right)_{j}(s)\right| \\
& \quad \leq \sum_{(J, E) \in \bar{\Gamma}_{j}^{(n)}} b_{(J, E)}(s)\left|f_{\alpha\left(j_{n}, e_{n}\right)}\left(\theta_{(J, E)}(s)\right)-g_{\alpha\left(j_{n}, e_{n}\right)}\left(\theta_{(J, E)}(s)\right)\right| .
\end{aligned}
$$

Using Lemma 3.5, there exists $\tau \in C_{\alpha\left(j_{n}, e_{n}\right)}$ with $d\left(\theta_{(J, E)}(s), \tau\right) \leq M_{1} c^{n}$.

Since $f_{\alpha\left(j_{n}, e_{n}\right)} \in K_{\alpha\left(j_{n}, e_{n}\right)}(M, \lambda)$ and $\|f\| \leq 1$, we have as in the proof of Lemma 3.2

$$
\left|f_{\alpha\left(j_{n}, e_{n}\right)}\left(\theta_{(J, E)}(s)\right)-f_{\alpha\left(j_{n}, e_{n}\right)}(\tau)\right| \leq M d\left(\theta_{(J, E)}(s), \tau\right)^{\lambda} \leq M\left(M_{1} c^{n}\right)^{\lambda} .
$$

The same is true for $g$. Also, if we assume that $f, g \in \mathcal{U}_{l}$ for some $l, 1 \leq l \leq q$, then $\left|f_{\alpha\left(j_{n}, e_{n}\right)}(\tau)-g_{\alpha\left(j_{n}, e_{n}\right)}(\tau)\right| \leq \epsilon$. Therefore, by the triangle inequality,

$$
\left|f_{\alpha\left(j_{n}, e_{n}\right)}\left(\theta_{(J, E)}(s)\right)-g_{\alpha\left(j_{n}, e_{n}\right)}\left(\theta_{(J, E)}(s)\right)\right| \leq \epsilon+2 M\left(M_{1} c^{n}\right)^{\lambda} \text { if } f, g \in \mathcal{U}_{l} .
$$

So, if $f, g \in \mathcal{U}_{l}$ and $1 \leq j \leq p$,

$$
\left|\left(A^{n} f\right)_{j}(s)-\left(A^{n} g\right)_{j}(s)\right| \leq\left(\epsilon+2 M\left(M_{1} c^{n}\right)^{\lambda}\right) \sum_{(J, E) \in \bar{\Gamma}_{j}^{(n)}} b_{(J, E)}(s) .
$$

Taking the supremum over $s \in S_{j}$ and the max over $1 \leq j \leq p$ and using (3.4), we get

$$
\left\|A^{n} f-A^{n} g\right\| \leq\left(\epsilon+2 M\left(M_{1} c^{n}\right)^{\lambda}\right)\left\|A^{n}\right\|
$$

for $f, g \in \mathcal{U}_{l}, 1 \leq l \leq q$.

Thus $A^{n}(\mathcal{U})=\bigcup_{l=1}^{q} A^{n}\left(\mathcal{U}_{l}\right)$ with

$$
\operatorname{diam}\left(A^{n}\left(\mathcal{U}_{l}\right)\right) \leq\left(\epsilon+2 M\left(M_{1} c^{n}\right)^{\lambda}\right)\left\|A^{n}\right\| .
$$

So, $\beta\left(A^{n}(\mathcal{U})\right) \leq\left(\epsilon+2 M\left(M_{1} c^{n}\right)^{\lambda}\right)\left\|A^{n}\right\|$.

Since $\epsilon>0$ was arbitrary, $\beta\left(A^{n}(\mathcal{U})\right) \leq 2 M\left(M_{1} c^{n}\right)^{\lambda}\left\|A^{n}\right\|$ which implies

$$
\left(\beta\left(A^{n}(\mathcal{U})\right)\right)^{\frac{1}{n}} \leq\left(2 M M_{1}^{\lambda}\right)^{\frac{1}{n}} c^{\lambda}\left\|A^{n}\right\|^{\frac{1}{n}} .
$$

In general, it is obviously true that $\left\|A^{n}\right\|_{K} \leq\left\|A^{n}\right\|$. On the other hand, if $f=\left(f_{1}, f_{2}, \ldots, f_{p}\right)$ and $f_{j}(s)=1$ for all $s \in S_{j}$, then $f \in K$, and we have seen in the proof of Lemma 3.4 that $\left\|A^{n}(f)\right\|=\left\|A^{n}\right\|$. It follows that $\left\|A^{n}\right\|_{K}=\left\|A^{n}\right\|$ for all $n \geq 1$ and that $r_{K}(A)=\lim _{n \rightarrow \infty}\left\|A^{n}\right\|^{\frac{1}{n}}=r(A)$, where $\left\|A^{n}\right\|$ is given by (3.4) and $r(A)$ denotes the spectral radius of $A$. Taking the limit in our estimate for $\left(\beta\left(A^{n}(\mathcal{U})\right)\right)^{\frac{1}{n}}$ gives

$$
\sigma_{K}(A)=\lim _{n \rightarrow \infty}\left(\beta\left(A^{n}(\mathcal{U})\right)\right)^{\frac{1}{n}} \leq\left(c^{\lambda}\right) r_{K}(A) .
$$

If $r_{K}(A)>0$, it follows (because $0<c<1$ ) that $\sigma_{K}(A)<r_{K}(A)$, and we are done. If we assume that $b_{(j, e)}(s)>0$ for all $(j, e) \in \Gamma$ and $s \in s_{j}$, then because $b_{(j, e)} \in K_{j}\left(M_{0}, \lambda\right)$, there exists $\delta>0$ such that $b_{(j, e)}(s) \geq \delta$ for all $(j, e) \in \Gamma$ and $s \in S_{j}$, and it follows easily that $r_{K}(A) \geq \delta>0$.

Remark 3.7. Suppose $u=\left(u_{1}, u_{2}, \ldots, u_{p}\right)$ is a nonzero eigenvector of the linear map $A$ with eigenvalue $r(A)$ given by Theorem 3.6. Then for any $1 \leq j \leq p$, either $u_{j}$ is identically zero or $u_{j}$ is strictly positive on $S_{j}$. To see this note that $u_{j} \in K_{j}(M, \lambda)$, so

$$
0 \leq u_{j}(s) \leq u_{j}(t) \exp \left(M\left(d_{j}(s, t)\right)^{\lambda}\right) \text { for all } s, t \in S_{j} .
$$


Thus $u_{j}(t)=0$ for some $t \in S_{j}$ will imply that $u_{j}(s)=0$ for all $s \in S_{j}$. Also, since $u$ is nonzero, at least one of the coordinate functions $u_{j}$ is strictly positive.

Remark 3.8. In general (in the context of the Krein-Rutman Theorem), if $r_{K}(L)=$ 0 , it need not be true that there exist $v \in K \backslash\{0\}$ with $L(v)=0$. Suppose, however, that $K$ and $A$ are as in Theorem 3.6, that $b_{(j, e)} \in K_{j}\left(M_{0}, \lambda\right)$ for all $(j, e) \in \Gamma$ and that $r_{K}(A)=0$. We claim that there exists an integer $N$ such that $A^{N}=0$ and that there exists $v \in K \backslash\{0\}$ with $A(v)=0$. Because $S_{j}$ is bounded and $b_{(j, e)} \in K_{j}\left(M_{0}, \lambda\right)$, we have already seen that either $b_{(j, e)}(s)=0$ for all $s \in S_{j}$ or there exists $\delta_{(j, e)}>0$ with $b_{(j, e)}(s) \geq \delta_{(j, e)}$ for all $s \in S_{j}$. Let $\mathcal{P}=\left\{(j, e) \in \Gamma: b_{(j, e)}(s)>0\right.$ for all $\left.s \in S_{j}\right\}$. Because $\mathcal{P}$ is a finite set (since $\Gamma$ is finite), there exists $\delta>0$ with $b_{(j, e)}(s) \geq \delta$ for all $s \in S_{j}$ and for all $(j, e) \in \mathcal{P}$. For $n \geq 1$, define $\overline{\mathcal{P}}^{(n)} \subset \bar{\Gamma}^{(n)}$ by

$$
\overline{\mathcal{P}}^{(n)}=\left\{(J, E) \in \bar{\Gamma}^{(n)}:\left(j_{k}, e_{k}\right) \in \mathcal{P} \text { for } 1 \leq k \leq n\right\} .
$$

If $\overline{\mathcal{P}}^{(n)}$ is nonempty, it easily follows from (3.4) that $\left\|A^{n}\right\| \geq \delta^{n}$, so if $\overline{\mathcal{P}}^{(n)}$ is nonempty for all $n \geq 1, r_{K}(A) \geq \delta$, contrary to our assumption. Thus there must exist an integer $N$ such that $\overline{\mathcal{P}}^{(n)}$ is empty for all $n \geq N$. However, if $(J, E) \in \bar{\Gamma}^{(n)}$ and $(J, E) \notin \overline{\mathcal{P}}^{(n)}, b_{(J, E)}(s)=0$ for all $s \in S_{j}$, so we find that $A^{n}=0$ for all $n \geq N$. If $w \in K \backslash\{0\}$, let $p \leq N$ be the least positive integer such that $A^{p}(w)=0$. If we define $v=A^{p-1}(w) \in K \backslash\{0\}, A(v)=0$.

Remark 3.9. If $\beta$ denotes the Kuratowski's measure of noncompactness on a Banach space $X, K$ denotes a closed cone in $X$ and $\Lambda: X \rightarrow X$ is a bounded linear map such that $\Lambda(K) \subset K$, define $\beta_{K}(\Lambda)$ by

$$
\beta_{K}(\Lambda):=\inf \{\lambda>0: \beta(\Lambda(T)) \leq \lambda \beta(T) \text { for all bounded sets } T \subset K\} .
$$

If $L: X \rightarrow X$ is a bounded linear map such that $L(K) \subset K$, one can prove that $\lim _{n \rightarrow \infty}\left(\beta_{K}\left(L^{n}\right)\right)^{\frac{1}{n}}$ exists. If $\lim _{n \rightarrow \infty}\left(\beta_{K}\left(L^{n}\right)\right)^{\frac{1}{n}}<r_{K}(L)$, Theorem 2.2 in [18] implies that there exists $u \in K \backslash\{0\}$ with $L(u)=r u$ and $r:=r_{K}(L)$.

One might hope that Theorem 2.2 in [18] could be used to prove Theorem 3.6 . However, if the metric spaces $S_{j}, 1 \leq j \leq p$, in Theorem [3.6 are not compact, Theorem 2.2 in 18 is, in general, not applicable, even in very simple special cases. To illustrate this we work in the Hilbert space $l^{2}(\mathbb{N})$, and we let $\left\{e_{j}: j \geq 1\right\}$ denote the usual orthonormal basis of $l^{2}(\mathbb{N})$. In the notation of Theorem 3.6. we take $S_{1}=\left\{x \in l^{2}(\mathbb{N}):\|x\| \leq 1\right\}, V=\{1\}$, and $\mathcal{E}$ to be a set with exactly one point. Let $X_{1}=C_{b}\left(S_{1}\right)$ and, in the notation of Lemma 3.2. let $K=K(M, 1)$, where $M$ is a fixed constant with $M \geq 1$. Define a bounded linear operator $A: X_{1} \rightarrow X_{1}$ by

$$
(A f)(x)=f\left(\frac{1}{2} x\right)
$$

for $x \in S_{1}$. Obviously, $A$ is a trivial example of the operators considered in Theorem [3.6, and $A(K(M, 1)) \subset K(M, 1)$ and $A u=u$, where $u \in K(M, 1)$ denotes the function identically equal to 1 . However, we claim that for each integer $n \geq 1$, there is a bounded set $T_{n} \subset K(M, 1)$ such that $\beta\left(A^{n}\left(T_{n}\right)\right)=\beta\left(T_{n}\right)=\left(\frac{1}{2}\right)^{n}$; this implies that $\lim _{n \rightarrow \infty}\left(\beta_{K}\left(A^{n}\right)\right)^{\frac{1}{n}}=1=r_{K}(A)$, so Theorem 2.2 of [18 cannot be used (at least with the cones $K(M, 1)$ ) even in this trivial situation.

To construct the sets $T_{n}$, for each integer $n \geq 1$ define $\phi_{n}:[0,1] \rightarrow\left[1,1+2^{-n}\right]$ by $\phi_{n}(s)=1+2^{-n}$ for $2^{-n} \leq s \leq 1$ and $\phi_{n}(s)=1+s$ for $0 \leq s \leq 2^{-n}$, so $\phi_{n}$ is 
Lipschitz with Lipschitz constant 1. Let $\langle\cdot, \cdot\rangle$ denote the inner product on $l^{2}(\mathbb{N})$, and define $\psi_{j, n}(x)$ and $T_{n}$ by

$$
\psi_{j, n}(x)=\phi_{n}\left(\left|\left\langle x, e_{j}\right\rangle\right|\right) \text { and } T_{n}=\left\{\psi_{j, n}: j \geq 1\right\} .
$$

The reader can verify that $T_{n} \subset K(M, 1)$ for all $n \geq 1$ and, that, for a fixed $n \geq 1$, and for $1 \leq j<k<\infty$,

$$
\left\|\psi_{j, n}-\psi_{k, n}\right\|=\sup \left\{\left|\psi_{j, n}(x)-\psi_{k, n}(x)\right|: x \in S_{1}\right\}=\left|\psi_{j, n}\left(e_{j}\right)-\psi_{k, n}\left(e_{j}\right)\right|=2^{-n} .
$$

Since $\left(A^{n} \psi_{j, n}\right)(x)=\phi_{n}\left(2^{-n}\left|\left\langle x, e_{j}\right\rangle\right|\right)$, the same argument shows that for a fixed $n \geq 1$ and for $1 \leq j<k<\infty$,

$$
\left\|A^{n}\left(\psi_{j, n}\right)-A^{n}\left(\psi_{k, n}\right)\right\|=\left|\psi_{j, n}\left(2^{-n} e_{j}\right)-\psi_{k, n}\left(2^{-n} e_{j}\right)\right|=2^{-n} .
$$

Using these equations, it is easy to verify that $\beta\left(T_{n}\right)=\beta\left(A^{n}\left(T_{n}\right)\right)=2^{-n}$.

\section{HAUSDORFF DIMENSION}

Recall that a metric space $(S, d)$ is called perfect if every point of $S$ is a limit point of $S$, i.e., for each $s \in S$, there exists a sequence $\left(s_{k}\right)_{k}$ in $S$ such that $s_{k} \neq s$ for all $k$ and $s_{k} \rightarrow s$ as $k \rightarrow \infty$.

Let $\left(S_{1}, d_{1}\right)$ and $\left(S_{2}, d_{2}\right)$ be perfect metric spaces. A map $\theta: S_{1} \rightarrow S_{2}$ is said to be an infinitesimal similitude at $s \in S_{1}$ if for any sequences $\left(s_{k}\right)_{k}$ and $\left(t_{k}\right)_{k}$ in $S_{1}$ with $s_{k} \neq t_{k}$ for $k \geq 1$ and $s_{k} \rightarrow s, t_{k} \rightarrow s$, the limit

$$
\lim _{k \rightarrow \infty} \frac{d_{2}\left(\theta\left(s_{k}\right), \theta\left(t_{k}\right)\right)}{d_{1}\left(s_{k}, t_{k}\right)}=:(D \theta)(s)
$$

exists and is independent of the particular sequences $\left(s_{k}\right)_{k}$ and $\left(t_{k}\right)_{k}$. We shall say that $\theta$ is an infinitesimal similitude on $S_{1}$ if $\theta$ is an infinitesimal similitude at $s$ for all $s \in S_{1}$. Notice that the assumption that $S_{1}$ is perfect implies that for every $s \in S_{1}$, there exist sequences $\left(s_{k}\right)_{k}$ and $\left(t_{k}\right)_{k}$ as above.

We list some basic properties of infinitesimal similitudes that we shall need.

Lemma 4.1. If $\theta: S_{1} \rightarrow S_{2}$ is an infinitesimal similitude, then $s \mapsto(D \theta)(s)$ is continuous.

Proof. We argue by contradiction and assume that $s \mapsto(D \theta)(s)$ is not continuous. Then there exist $\epsilon>0$ and $s \in S_{1}$ and a sequence $\left(s_{k}\right)_{k}$ in $S_{1}$ with $d_{1}\left(s_{k}, s\right) \rightarrow 0$ as $k \rightarrow \infty$ such that

$$
\left|(D \theta)\left(s_{k}\right)-(D \theta)(s)\right|>\epsilon>0 .
$$

Since $S_{1}$ is perfect and $\theta$ is an infinitesimal similitude, for each $k \geq 1$, there exist $t_{k}$ and $w_{k}$ in $S_{1}$ with $t_{k} \neq w_{k}, 0<d_{1}\left(s_{k}, t_{k}\right)<\frac{1}{k}, 0<d_{1}\left(s_{k}, w_{k}\right)<\frac{1}{k}$ and

$$
\left|\frac{d_{2}\left(\theta\left(t_{k}\right), \theta\left(w_{k}\right)\right)}{d_{1}\left(t_{k}, w_{k}\right)}-(D \theta)\left(s_{k}\right)\right|<\frac{\epsilon}{4} \text {. }
$$

Since $s_{k} \rightarrow s$ as $k \rightarrow \infty$, it follows that $t_{k} \rightarrow s$ and $w_{k} \rightarrow s$ as $k \rightarrow \infty$ as well. So, by definition,

$$
\frac{d_{2}\left(\theta\left(t_{k}\right), \theta\left(w_{k}\right)\right)}{d_{1}\left(t_{k}, w_{k}\right)} \rightarrow(D \theta)(s) \text { as } k \rightarrow \infty .
$$

So, for $k$ large enough, $\left|(D \theta)\left(s_{k}\right)-(D \theta)(s)\right|<\frac{\epsilon}{2}$, a contradiction. Hence, $s \mapsto$ $(D \theta)(s)$ is continuous.

The following lemma states an analogue of the "chain rule" for infinitesimal similitudes. 
Lemma 4.2. Let $\theta: S_{1} \rightarrow S_{2}$ and $\psi: S_{2} \rightarrow S_{3}$ be given. If $\theta$ is an infinitesimal similitude at $s \in S_{1}$ and $\psi$ is an infinitesimal similitude at $\theta(s) \in S_{2}$, then $\psi \circ \theta$ is an infinitesimal similitude at $s$ and

$$
(D(\psi \circ \theta))(s)=(D \psi)(\theta(s))(D \theta)(s) .
$$

Proof. Let $\left(s_{k}\right)_{k}$ and $\left(t_{k}\right)_{k}$ be sequences in $S_{1}$ with $s_{k} \neq t_{k}, k \geq 1, s_{k} \rightarrow s, t_{k} \rightarrow s$ as $k \rightarrow \infty$. Then $\theta\left(s_{k}\right) \rightarrow \theta(s), \theta\left(t_{k}\right) \rightarrow \theta(s)$ as $k \rightarrow \infty$. We consider two cases.

Case I. Assume that $(D \theta)(s) \neq 0$. We claim that there exists a positive integer $k_{0}$ with $\theta\left(s_{k}\right) \neq \theta\left(t_{k}\right)$ for all $k \geq k_{0}$. If not, there exists a subsequence $k_{i} \rightarrow \infty$ such that $\theta\left(s_{k_{i}}\right)=\theta\left(t_{k_{i}}\right)$ for $i \geq 1$. Writing $\sigma_{i}=s_{k_{i}}$ and $\tau_{i}=t_{k_{i}}$, we have that $\sigma_{i} \rightarrow s, \tau_{i} \rightarrow s, \sigma_{i} \neq \tau_{i}$ and $(D \theta)(s)=\lim _{i \rightarrow \infty} \frac{d_{2}\left(\theta\left(\sigma_{i}\right), \theta\left(\tau_{i}\right)\right)}{d_{1}\left(\sigma_{i}, \tau_{i}\right)}=0$, which contradicts our assumption. It follows that, for $k \geq k_{0}$, we can write

$$
\frac{d_{3}\left(\psi\left(\theta\left(s_{k}\right)\right), \psi\left(\theta\left(t_{k}\right)\right)\right)}{d_{1}\left(s_{k}, t_{k}\right)}=\frac{d_{3}\left(\psi\left(\theta\left(s_{k}\right)\right), \psi\left(\theta\left(t_{k}\right)\right)\right)}{d_{2}\left(\theta\left(s_{k}\right), \theta\left(t_{k}\right)\right)} \frac{d_{2}\left(\theta\left(s_{k}\right), \theta\left(t_{k}\right)\right)}{d_{1}\left(s_{k}, t_{k}\right)} .
$$

As $k \rightarrow \infty$, the limit of the right-hand side exists and equals $(D \psi)(\theta(s))(D \theta)(s)$, so the limit of the left-hand side exists and (4.2) is satisfied.

Notice that if $s_{k}$ and $t_{k}$ are sequences with $s_{k} \rightarrow s, t_{k} \rightarrow s, s_{k} \neq t_{k}$ for all $k$ and $\theta\left(s_{k}\right) \neq \theta\left(t_{k}\right)$ for all $k \geq k_{0}$, then the argument above proves that

$$
\lim _{k \rightarrow \infty} \frac{d_{3}\left(\psi\left(\theta\left(s_{k}\right)\right), \psi\left(\theta\left(t_{k}\right)\right)\right)}{d_{1}\left(s_{k}, t_{k}\right)}=(D \psi)(\theta(s))(D \theta)(s)
$$

even if $(D \theta)(s)=0$.

Case II. Assume that $(D \theta)(s)=0$. Let $s_{k}$ and $t_{k}$ be sequences in $S_{1}$ with $s_{k} \rightarrow s$, $t_{k} \rightarrow s$ and $s_{k} \neq t_{k}$ for all $k \geq 1$. If there exists $k_{0} \geq 1$ such that $\theta\left(s_{k}\right) \neq \theta\left(t_{k}\right)$ for all $k \geq k_{0}$, the argument above shows that

$$
\lim _{k \rightarrow \infty} \frac{d_{3}\left(\psi\left(\theta\left(s_{k}\right)\right), \psi\left(\theta\left(t_{k}\right)\right)\right)}{d_{1}\left(s_{k}, t_{k}\right)}=(D \psi)(\theta(s))(D \theta)(s)=0 .
$$

If there exists $k_{1}$ such that $\theta\left(s_{k}\right)=\theta\left(t_{k}\right)$ for all $k \geq k_{1}$, we certainly have that

$$
\lim _{k \rightarrow \infty} \frac{d_{3}\left(\psi\left(\theta\left(s_{k}\right)\right), \psi\left(\theta\left(t_{k}\right)\right)\right)}{d_{1}\left(s_{k}, t_{k}\right)}=0 .
$$

Thus we can assume that $K_{1}:=\left\{k \geq 1: \theta\left(s_{k}\right) \neq \theta\left(t_{k}\right)\right\}$ and $K_{2}:=\left\{k \geq 1: \theta\left(s_{k}\right)=\right.$ $\left.\theta\left(t_{k}\right)\right\}$ are infinite sets. However, our previous argument (Case I) shows that

$$
\lim _{k \rightarrow \infty, k \in K_{1}} \frac{d_{3}\left(\psi\left(\theta\left(s_{k}\right)\right), \psi\left(\theta\left(t_{k}\right)\right)\right)}{d_{1}\left(s_{k}, t_{k}\right)}=(D \psi)(\theta(s))(D \theta)(s)=0,
$$

and it is clear that

$$
\lim _{k \rightarrow \infty, k \in K_{2}} \frac{d_{3}\left(\psi\left(\theta\left(s_{k}\right)\right), \psi\left(\theta\left(t_{k}\right)\right)\right)}{d_{1}\left(s_{k}, t_{k}\right)}=0,
$$

so we conclude that

$$
\lim _{k \rightarrow \infty} \frac{d_{3}\left(\psi\left(\theta\left(s_{k}\right)\right), \psi\left(\theta\left(t_{k}\right)\right)\right)}{d_{1}\left(s_{k}, t_{k}\right)}=0
$$

The following lemma gives a "mean value theorem" and will be crucial in the proof of the main theorem. 
Lemma 4.3. Suppose that $\left(S_{1}, d_{1}\right)$ and $\left(S_{2}, d_{2}\right)$ are bounded, complete metric spaces and that $\theta: S_{1} \rightarrow S_{2}$ is an infinitesimal similitude. Also, assume that $\theta$ is Lipschitz and that $(D \theta)(s)>0$ for all $s \in S_{1}$. Let $K \subset S_{1}$ be a compact, nonempty set. Then for each $\mu>1$, there exists an open neighborhood $U_{\mu}$ of $K$ and a positive number $\epsilon=\epsilon(\mu)$ such that for every $s, t \in U_{\mu}$ with $0<d_{1}(s, t)<\epsilon(\mu)$,

$$
\mu^{-1}(D \theta)(s) \leq \frac{d_{2}(\theta(s), \theta(t))}{d_{1}(s, t)} \leq \mu(D \theta)(s) .
$$

Proof. For $(s, t) \in S_{1} \times S_{1}$ with $s \neq t$, define $F(s, t)=\frac{d_{2}(\theta(s), \theta(t))}{d_{1}(s, t)}$. If $(s, s) \in S_{1} \times S_{1}$, define $F(s, s)=(D \theta)(s)$. Because we assume that $\theta$ is Lipschitz on $S_{1}$, there is a constant $M_{1}$ with $F(s, t) \leq M_{1}$ for all $(s, t) \in S_{1} \times S_{1}$.

We claim that $F$ is continuous on $S_{1} \times S_{1}$. It suffices to prove that if $\left(s_{k}, t_{k}\right) \rightarrow$ $(s, s)$, then $F\left(s_{k}, t_{k}\right) \rightarrow F(s, s)=(D \theta)(s)$. If $s_{k} \neq t_{k}$ for all $k \geq k_{0}$, we know that $F\left(s_{k}, t_{k}\right) \rightarrow(D \theta)(s)$ by the definition of $(D \theta)(s)$. If $s_{k}=t_{k}$ for all $k \geq k_{1}$, then $F\left(s_{k}, t_{k}\right)=(D \theta)\left(s_{k}\right)$ for $k \geq k_{1}$, and Lemma 4.1 implies that $(D \theta)\left(s_{k}\right) \rightarrow(D \theta)(s)$. Thus we can assume that $J_{1}:=\left\{k: s_{k} \neq t_{k}\right\}$ and $J_{2}:=\left\{k: s_{k}=t_{k}\right\}$ are infinite sets. But in this case, the same reasoning implies that $\lim _{k \rightarrow \infty, k \in J_{1}} F\left(s_{k}, t_{k}\right)=$ $(D \theta)(s)$ and $\lim _{k \rightarrow \infty, k \in J_{2}} F\left(s_{k}, t_{k}\right)=(D \theta)(s)$, so $\lim _{k \rightarrow \infty} F\left(s_{k}, t_{k}\right)=(D \theta)(s)$.

Lemma 4.1 implies that $s \mapsto(D \theta)(s)$ is continuous on $S_{1}$, and $(s, t) \mapsto F(s, t)$ is continuous on $S_{1} \times S_{1}$. Thus, since $(D \theta)(s)>0$, if we define $G(s, t)$ by $G(s, t)=$ $\frac{F(s, t)}{(D \theta)(s)},(s, t) \mapsto G(s, t)$ is continuous on $S_{1} \times S_{1}$ and $G(s, s)=1$. Since $K \times K$ is compact, $\left.G\right|_{K \times K}$ is uniformly continuous, so given $\mu>1$, there exists $\epsilon(\mu)>0$ with

$$
\mu^{-1}<G(s, t)<\mu
$$

for all $(s, t) \in K \times K$ with $d_{1}(s, t) \leq \epsilon(\mu)$. We claim that there exists an open neighborhood $U_{\mu}$ of $K$ such that for all $s, t \in U_{\mu}$ with $d_{1}(s, t) \leq \epsilon(\mu), \mu^{-1}<$ $G(s, t)<\mu$. We argue by contradiction and suppose not. For $m$ a positive integer, let $V_{m}=\left\{s \in S_{1}: d_{1}(s, K)<\frac{1}{m}\right\}$. By assumption, there exist $s_{m}, t_{m} \in V_{m}$ with $d_{1}\left(s_{m}, t_{m}\right) \leq \epsilon(\mu)$ and $G\left(s_{m}, t_{m}\right)<\mu^{-1}$ or $G\left(s_{m}, t_{m}\right)>\mu$. Because $d_{1}\left(s_{m}, K\right) \rightarrow 0$ and $d_{1}\left(t_{m}, K\right) \rightarrow 0$, we can, by taking a subsequence, assume that $s_{m} \rightarrow s \in K$ and $t_{m} \rightarrow t \in K$ and $d_{1}(s, t) \leq \epsilon(\mu)$. By continuity of $G$, we either have $G(s, t) \leq \mu^{-1}$ or $G(s, t) \geq \mu$. However, because $s, t \in K$ and $d_{1}(s, t) \leq \epsilon(\mu), \mu^{-1}<G(s, t)<\mu$, a contradiction. Thus an open set $U_{\mu}$ exists and, in fact, we can take $U_{\mu}=V_{m}$ for some $m \geq 1$.

Remark 4.4. For each $\epsilon>0$, define $\mu(\epsilon) \geq 1$ to be the infimum of numbers $\mu>1$ such that $\mu^{-1}(D \theta)(s) \leq \frac{d_{2}(\theta(s), \theta(t))}{d_{1}(s, t)} \leq \mu(D \theta)(s)$ for $s, t \in K$ with $0<d_{1}(s, t) \leq \epsilon$. Lemma 4.3 implies that $\lim _{\epsilon \rightarrow 0+} \mu(\epsilon)=1$, and clearly $\mu(\epsilon)$ is an increasing function of $\epsilon$ for $\epsilon>0$.

Throughout this section we shall make the following assumption.

H4.1 Let $V=\{1,2, \ldots, p\}$ and $S_{1}, S_{2}, \ldots, S_{p}$ be bounded, complete, perfect metric spaces. Let $\mathcal{E}$ be a finite set, $\Gamma \subset V \times \mathcal{E}$ and $\alpha: \Gamma \rightarrow V$. For each $(j, e) \in \Gamma, \theta_{(j, e)}: S_{j} \rightarrow S_{\alpha(j, e)}$ is a Lipschitz map with $\operatorname{Lip}\left(\theta_{(j, e)}\right) \leq c<1$. Also, $\Gamma_{i}=\{(j, e) \in \Gamma: \alpha(j, e)=i\} \neq \emptyset$ for $1 \leq i \leq p$ and $E_{j}=\{e \in \mathcal{E}:(j, e) \in \Gamma\} \neq \emptyset$ for $1 \leq j \leq p$. 
If H4.1 is satisfied then Theorem 2.3 implies that there exists a unique list of nonempty compact sets $C_{j} \subset S_{j}, 1 \leq j \leq p$ with

$$
C_{i}=\bigcup_{(j, e) \in \Gamma_{i}} \theta_{(j, e)}\left(C_{j}\right) \quad \text { for } 1 \leq i \leq p .
$$

We shall further assume the following.

H4.2 For each $(j, e) \in \Gamma$, the map $\theta_{(j, e)}: S_{j} \rightarrow S_{\alpha(j, e)}$, given in H4.1, is an infinitesimal similitude and $\left(D \theta_{(j, e)}\right)(s)>m>0$ for all $s \in S_{j}$.

Notice that since $\theta_{(j, e)}$ is $\operatorname{Lipschitz}$ with $\operatorname{Lip}\left(\theta_{(j, e)}\right) \leq c$, if $\theta_{(j, e)}$ is an infinitesimal similitude, we have $\left(D \theta_{(j, e)}\right)(s) \leq c$.

Assume that H4.1 and H4.2 are satisfied. For $\sigma \geq 0$, define

$$
L_{\sigma}: \prod_{j=1}^{p} C_{b}\left(S_{j}\right) \rightarrow \prod_{j=1}^{p} C_{b}\left(S_{j}\right)
$$

by

$$
\left(L_{\sigma} f\right)_{j}(s)=\sum_{e \in E_{j}}\left(\left(D \theta_{(j, e)}\right)(s)\right)^{\sigma} f_{\alpha(j, e)}\left(\theta_{(j, e)}(s)\right) \text { for } s \in S_{j}, 1 \leq j \leq p .
$$

Recall that a map $f:(S, d) \rightarrow \mathbb{R}$ is said to be Hölder continuous with Hölder exponent $\lambda>0$ if there exists a constant $C \in(0, \infty)$ such that

$$
|f(s)-f(t)| \leq C(d(s, t))^{\lambda} \text { for all } s, t \in S .
$$

Let us assume the following.

H4.3 For each $(j, e) \in \Gamma$, the map $s \mapsto\left(D \theta_{(j, e)}\right)(s)$ is Hölder continuous with Hölder exponent $\lambda>0$, where $\lambda$ is independent of $(j, e) \in \Gamma$.

Lemma 4.5. If $\mathrm{H} 4.1, \mathrm{H} 4.2$ and $\mathrm{H} 4.3$ hold, and $\sigma \geq 0$, then the map

$$
s \mapsto\left(\left(D \theta_{(j, e)}\right)(s)\right)^{\sigma}
$$

is in the cone $K_{j}\left(M_{0}, \lambda\right)$ defined by (3.2) for some $M_{0}>0$ (depending on $\sigma$ ).

Proof. Fix $(j, e) \in \Gamma$ and $\sigma \geq 0$. Let $f(s)=\left(D \theta_{(j, e)}\right)(s)$. The hypotheses H4.1 and H4.2 imply that $0<m<f(s) \leq c$. By H4.3,

$$
|f(s)-f(t)| \leq C\left(d_{j}(s, t)\right)^{\lambda} \text { for all } s, t \in S_{j} .
$$

Let $s, t \in S_{j}$. By the mean value theorem, there exists $\xi$ between $f(s)$ and $f(t)$ such that

$$
|\ln (f(s))-\ln (f(t))|=\frac{1}{\xi}|f(s)-f(t)| \leq \frac{1}{\xi} C\left(d_{j}(s, t)\right)^{\lambda} \leq \frac{1}{m} C\left(d_{j}(s, t)\right)^{\lambda} .
$$

So, $f(s) \leq f(t) \exp \left(\frac{1}{m} C\left(d_{j}(s, t)\right)^{\lambda}\right)$, which implies that

$$
f(s)^{\sigma} \leq f(t)^{\sigma} \exp \left(M_{0}\left(d_{j}(s, t)\right)^{\lambda}\right), \text { where } M_{0}=\frac{\sigma C}{m} .
$$

This completes the proof.

Now applying Theorem 3.6 to the linear map $L_{\sigma}$ defined in (4.4), we get an eigenvector $u_{\sigma} \in K \backslash\{0\}$ with $L_{\sigma} u_{\sigma}=r\left(L_{\sigma}\right) u_{\sigma}$ and $r\left(L_{\sigma}\right)>0$.

Lemma 4.6. The map $\sigma \mapsto r\left(L_{\sigma}\right)$ is continuous and strictly decreasing. Furthermore, there is a unique $\sigma_{0} \geq 0$ such that $r\left(L_{\sigma_{0}}\right)=1$. 
Proof. Let $u_{\sigma}$ be the positive eigenvector of $L_{\sigma}$ with eigenvalue $r\left(L_{\sigma}\right)$. Let us write $b_{(j, e)}(t)=\left(D \theta_{(j, e)}(t)\right)$ for $(j, e) \in \Gamma$. We know that $0<m \leq b_{(j, e)}(t) \leq c<1$ for all $t \in S_{j}$. Let $0 \leq \sigma<\sigma^{\prime}$. Then

$$
\left(b_{(j, e)}(t)\right)^{\sigma^{\prime}}=\left(b_{(j, e)}(t)\right)^{\sigma^{\prime}-\sigma}\left(b_{(j, e)}(t)\right)^{\sigma} \leq c^{\sigma^{\prime}-\sigma}\left(b_{(j, e)}(t)\right)^{\sigma} .
$$

Therefore, $\left(b_{(j, e)}(t)\right)^{\sigma} \geq \mu\left(b_{(j, e)}(t)\right)^{\sigma^{\prime}}$, where $\mu=\left(\frac{1}{c}\right)^{\sigma^{\prime}-\sigma}>1$. It follows that for all $t \in S_{j}$,

$$
\begin{aligned}
\left(L_{\sigma} u_{\sigma^{\prime}}\right)_{j}(t) & =\sum_{e \in E_{j}}\left(b_{(j, e)}(t)\right)^{\sigma}\left(u_{\sigma^{\prime}}\right)_{\alpha(j, e)}\left(\theta_{(j, e)}(t)\right) \\
& \geq \mu \sum_{e \in E_{j}}\left(b_{(j, e)}(t)\right)^{\sigma^{\prime}}\left(u_{\sigma^{\prime}}\right)_{\alpha(j, e)}\left(\theta_{(j, e)}(t)\right) \\
& =\mu\left(L_{\sigma}^{\prime} u_{\sigma^{\prime}}\right)_{j}(t)=\mu r\left(L_{\sigma^{\prime}}\right)\left(u_{\sigma^{\prime}}\right)_{j}(t),
\end{aligned}
$$

so $L_{\sigma} u_{\sigma^{\prime}} \geq \mu r\left(L_{\sigma^{\prime}}\right) u_{\sigma^{\prime}}$, where the inequality has the natural coordinate-wise interpretation. Iterating this inequality $k$ times, we obtain

$$
L_{\sigma}^{k} u_{\sigma^{\prime}} \geq\left(\mu r\left(L_{\sigma^{\prime}}\right)\right)^{k} u_{\sigma^{\prime}} .
$$

If $e$ denotes the function identically equal to one in each component, we have $u_{\sigma^{\prime}} \leq\left\|u_{\sigma^{\prime}}\right\| e$. Thus

$$
L_{\sigma}^{k} u_{\sigma^{\prime}} \leq L_{\sigma}^{k}\left(\left\|u_{\sigma^{\prime}}\right\| e\right) \leq\left\|u_{\sigma^{\prime}}\right\| L_{\sigma}^{k}(e) .
$$

Taking norms, we get

$$
\left\|u_{\sigma^{\prime}}\right\|\left\|L_{\sigma}^{k}(e)\right\| \geq\left\|L_{\sigma}^{k} u_{\sigma^{\prime}}\right\| \geq\left(\mu r\left(L_{\sigma^{\prime}}\right)\right)^{k}\left\|u_{\sigma^{\prime}}\right\| .
$$

So, $\left\|L_{\sigma}^{k}\right\| \geq\left\|L_{\sigma}^{k}(e)\right\| \geq\left(\mu r\left(L_{\sigma^{\prime}}\right)\right)^{k}$, from which it follows that

$$
r\left(L_{\sigma}\right)=\lim _{k \rightarrow \infty}\left\|L_{\sigma}^{k}\right\|^{1 / k} \geq \mu r\left(L_{\sigma^{\prime}}\right) .
$$

Since $\mu>1$, we have proved that $r\left(L_{\sigma}\right)>r\left(L_{\sigma^{\prime}}\right)$.

Next we prove the continuity of $\sigma \mapsto r\left(L_{\sigma}\right)$. Let $\sigma>0$ be fixed. Given $\nu<1$, select $\delta>0$ such that

$$
\nu\left(b_{(j, e)}(t)\right)^{\sigma} \leq\left(b_{(j, e)}(t)\right)^{\sigma^{\prime}} \leq \nu^{-1}\left(b_{(j, e)}(t)\right)^{\sigma} \text { for } t \in S_{j},\left|\sigma-\sigma^{\prime}\right| \leq \delta .
$$

Then, using the argument as above, we have $\nu r\left(L_{\sigma}\right) \leq r\left(L_{\sigma^{\prime}} \leq \nu^{-1} r\left(L_{\sigma}\right)\right.$ whenever $\left|\sigma-\sigma^{\prime}\right| \leq \delta$. Since $\nu<1$ was arbitrary, this proves that $\sigma \mapsto r\left(L_{\sigma}\right)$ is continuous.

Since $\left\|L_{0}^{k}\right\| \geq\left\|L_{0}^{k} e\right\| \geq 1$, we see that $r\left(L_{0}\right) \geq 1$. Also if $|\mathcal{E}|$ denotes the cardinality of $\mathcal{E}$, then from the definition of $L_{\sigma}$ and using the fact that $D_{(j, e)}(t) \leq c$ for all $t \in S_{j}$ and $(j, e) \in \Gamma$, it is clear that $\left\|L_{\sigma}\right\| \leq|\mathcal{E}| c^{\sigma} \rightarrow 0$ as $\sigma \rightarrow \infty$. So $r\left(L_{\sigma}\right) \leq\left\|L_{\sigma}\right\| \rightarrow 0$ as $\sigma \rightarrow \infty$. It follows by the continuity and strict monotonicity of $\sigma \rightarrow r\left(L_{\sigma}\right)$ that there exists a unique $\sigma_{0} \geq 0$ such that $r\left(L_{\sigma_{0}}\right)=1$.

Definition 4.7. We define strong connectedness to be the property that for each pair $j$ and $k$ in $V$, there exists for some $n \geq 1,(J, E)=\left[\left(j_{1}, e_{1}\right), \ldots,\left(j_{n}, e_{n}\right)\right]$ such that $\left(j_{i}, e_{i}\right) \in \Gamma$, for $1 \leq i \leq n, j_{1}=j, \alpha\left(j_{i}, e_{i}\right)=j_{i+1}, 1 \leq i<n$ and $\alpha\left(j_{n}, e_{n}\right)=k$. Note that in this case we have a map $\theta_{(J, E)}=\theta_{\left(j_{n}, e_{n}\right)} \circ \cdots \circ \theta_{\left(j_{1}, e_{1}\right)}$ which maps $S_{j}$ into $S_{k}$. Note also (compare H4.1) that strong connectedness implies that $\Gamma_{i} \neq \emptyset$ for $1 \leq i \leq p$ and $E_{j} \neq \emptyset$ for $1 \leq j \leq p$.

From now on we shall always assume strong connectedness.

H4.4 The property of strong connectedness is satisfied. 
Lemma 4.8. Assume that the hypotheses $\mathrm{H} 4.1, \mathrm{H} 4.2, \mathrm{H} 4.3$ and $\mathrm{H} 4.4$ are satisfied and let $u_{\sigma} \in K \backslash\{0\}$ be a nonzero eigenvector of $L_{\sigma}$ with eigenvalue $r\left(L_{\sigma}\right)$. Then each component $\left(u_{\sigma}\right)_{j}$ is a strictly positive function on $S_{j}$ for $1 \leq j \leq p$. Furthermore, there are constants $l$ and $L$ with $0<l \leq L<\infty$ such that for every $j$, $1 \leq j \leq p$,

$$
l \leq\left(u_{\sigma}\right)_{j}(t) \leq L \quad \text { for all } t \in S_{j} .
$$

Proof. Suppose for some $j, 1 \leq j \leq p$, that $\left(u_{\sigma}\right)_{j}$ equals zero at some point in $S_{j}$. Then, since $\left(u_{\sigma}\right)_{j} \in K_{j}(M, \lambda)$, it follows, as shown in Remark 2.2, that $\left(u_{\sigma}\right)_{j}$ is identically equal to zero on $S_{j}$. Fix a $k, 1 \leq k \leq p$. By strong connectedness, there exist $n \geq 1$ and $\left(J^{\prime}, E^{\prime}\right)=\left[\left(j_{1}, e_{1}\right), \ldots,\left(j_{n}, e_{n}\right)\right] \in \bar{\Gamma}_{j}^{(n)}$ with $j_{1}=j$ and $\alpha\left(j_{n}, e_{n}\right)=k$. Since $L_{\sigma} u_{\sigma}=r\left(L_{\sigma}\right) u_{\sigma}$, it follows that $L_{\sigma}^{n} u_{\sigma}=\left(r\left(L_{\sigma}\right)\right)^{n} u_{\sigma}$. So, using the formula for $L_{\sigma}^{n}$ given by Lemma 3.4 with $A$ replaced by $L_{\sigma}$, we get

$$
\left(r\left(L_{\sigma}\right)\right)^{n}\left(u_{\sigma}\right)_{j}(s)=\sum_{(J, E) \in \bar{\Gamma}_{j}^{(n)}} b_{(J, E)}(s)\left(u_{\sigma}\right)_{\alpha\left(j_{n}, e_{n}\right)}\left(\theta_{(J, E)}(s)\right),
$$

where $b_{(j, e)}(s)=\left(\left(D \theta_{(j, e)}\right)(s)\right)^{\sigma}$. The left-hand side in the above equation is zero because $\left(u_{\sigma}\right)_{j}(s)=0$. Thus, since each term in the sum on the right-hand side is nonnegative, it follows that each term equals zero. In particular, $\left(u_{\sigma}\right)_{k}\left(\theta_{(J, E)}(s)\right)=$ 0 since $b_{(J, E)}(s)$ is strictly positive by H4.2. This implies that $\left(u_{\sigma}\right)_{k}$ is identically equal to zero on $S_{k}$. Since this is true for any $k, 1 \leq k \leq p$, we arrive at a contradiction that $u_{\sigma}$ is identically zero. Thus $\left(u_{\sigma}\right)_{j}$ is a strictly positive function on $S_{j}$ for $1 \leq j \leq p$.

Since each $S_{j}$ is bounded, there is a $D<\infty$ such that $\operatorname{diam}\left(S_{j}\right) \leq D$ for $1 \leq$ $j \leq p$. Then, since $\left(u_{\sigma}\right)_{j} \in K_{j}(M, \lambda)$, it follows that

$$
0<\left(u_{\sigma}\right)_{j}(s) \leq\left(u_{\sigma}\right)_{j}(t) \exp \left(M D^{\lambda}\right) \text { for all } s, t \in S_{j} .
$$

From this it is easy to see that there are constants $0<l \leq L<\infty$ such that

$$
l \leq\left(u_{\sigma}\right)_{j}(t) \leq L \text { for all } t \in S_{j}, 1 \leq j \leq p .
$$

Let $C_{j} \subset S_{j}, 1 \leq j \leq p$, be the invariant list of nonempty compact sets such that

$$
C_{i}=\bigcup_{(j, e) \in \Gamma_{i}} \theta_{(j, e)}\left(C_{j}\right) \text { for } 1 \leq i \leq p
$$

Our goal is to determine the Hausdorff dimension of the sets $C_{i}, 1 \leq i \leq p$.

Let us recall the definition of Hausdorff measure and Hausdorff dimension. Suppose $(X, d)$ is a metric space and $A \subset X$. We define, for $\epsilon>0$ and $\sigma \geq 0$,

$$
\mathcal{H}_{\epsilon}^{\sigma}(A)=\inf \left\{\sum_{k=1}^{\infty}\left(\operatorname{diam}\left(A_{k}\right)\right)^{\sigma}: A \subset \bigcup_{k=1}^{\infty} A_{k}, \operatorname{diam}\left(A_{k}\right)<\epsilon\right\} .
$$

It follows that $\mathcal{H}_{\epsilon}^{\sigma}$ is an outer measure. For a given $\sigma \geq 0$, the function $\epsilon \mapsto \mathcal{H}_{\epsilon}^{\sigma}$ is decreasing and we define

$$
\mathcal{H}^{\sigma}(A)=\lim _{\epsilon \rightarrow 0+} \mathcal{H}_{\epsilon}^{\sigma}(A)=\sup _{\epsilon>0} \mathcal{H}_{\epsilon}^{\sigma}(A) .
$$

It follows that $\mathcal{H}^{\sigma}$ is a Borel measure and is called Hausdorff $\sigma$-dimensional measure. It is not hard to prove that there is a unique number $\sigma_{0} \geq 0$ such that $\mathcal{H}^{\sigma}(A)=$ 
$\infty$ for $0 \leq \sigma<\sigma_{0}$ and $\mathcal{H}^{\sigma}(A)=0$ for $\sigma>\sigma_{0}$. The number $\sigma_{0}$ is called the Hausdorff dimension of $A$. We refer the reader to [5] and [11] for the basic properties of Hausdorff measure.

First we shall prove that the Hausdorff dimension of $C_{i}$ is independent of $i$, $1 \leq i \leq p$, under the assumption of strong connectedness.

Lemma 4.9. Assume that the hypotheses $\mathrm{H} 4.1, \mathrm{H} 4.2, \mathrm{H} 4.3$ and $\mathrm{H} 4.4$ are satisfied and let $C_{j} \subset S_{j}, 1 \leq j \leq p$, be the unique invariant list of compact, nonempty sets such that

$$
C_{i}=\bigcup_{(j, e) \in \Gamma_{i}} \theta_{(j, e)}\left(C_{j}\right) \text { for } 1 \leq i \leq p .
$$

Then $\operatorname{dim}\left(C_{j}\right)$, the Hausdorff dimension of $C_{j}$, is independent of $j$ for $1 \leq j \leq p$.

Proof. First we claim that $\operatorname{dim}\left(\theta_{(j, e)}\left(C_{j}\right)\right)=\operatorname{dim}\left(C_{j}\right)$ for any $(j, e) \in \Gamma$. Since $\theta_{(j, e)}$ is a Lipschitz map with Lipschitz constant $c, \mathcal{H}^{\sigma}\left(\theta_{(j, e)}\left(C_{j}\right)\right) \leq c^{\sigma} \mathcal{H}^{\sigma}\left(C_{j}\right)$ for any $\sigma \geq 0$. This implies that $\operatorname{dim}\left(\theta_{(j, e)}\left(C_{j}\right)\right) \leq \operatorname{dim}\left(C_{j}\right)$. To prove the other inequality, we first claim that there exist $m_{0}>0$ and $\delta>0$ such that $d\left(\theta_{(j, e)}(s), \theta_{(j, e)}(t)\right) \geq m_{0} d(s, t)$ for all $s, t \in C_{j}$ with $d(s, t) \leq \delta$. We abuse notation here by letting $d$ denote $d_{j}$ and $d_{\alpha(j, e)}$. We argue by contradiction. If the claim is false, then for each positive integer $k$ there exist $s_{k}, t_{k} \in C_{j}$ with $0<d\left(s_{k}, t_{k}\right) \leq k^{-1}$ and $d\left(\theta_{(j, e)}\left(s_{k}\right), \theta_{(j, e)}\left(t_{k}\right)\right)<k^{-1} d\left(s_{k}, t_{k}\right)$. Since $C_{j}$ is compact, by taking a subsequence, we can assume that $s_{k} \rightarrow s$ and $t_{k} \rightarrow s$. But this implies that $D \theta_{(j, e)}(s)=0$, which contradicts H4.2. Thus $m_{0}>0$ and $\delta>0$ exist, and since $C_{j}$ is compact, we can write $C_{j}=\bigcup_{l=1}^{p} C_{j, l}$, where $\operatorname{diam}\left(C_{j, l}\right) \leq \delta$ and $p<\infty$. It follows easily from the definition of Hausdorff dimension that there exists $l$ such that the Hausdorff dimension of $C_{j, l}$ equals the Hausdorff dimension of $C_{j}$. Also, by our construction, $\left.\theta_{(j, e)}\right|_{C_{j, l}}$ is one-to-one and $\left(\left.\theta_{(j, e)}\right|_{C_{j, l}}\right)^{-1}$ is Lipschitian. This implies that

$$
\operatorname{dim}\left(C_{j}\right)=\operatorname{dim}\left(C_{j, l}\right) \leq \operatorname{dim}\left(\theta_{(j, e)}\left(C_{j, l}\right)\right) \leq \operatorname{dim}\left(\theta_{(j, e)}\left(C_{j}\right)\right),
$$

and we have shown that $\operatorname{dim}\left(C_{j}\right)=\operatorname{dim}\left(\theta_{(j, e)}\left(C_{j}\right)\right)$.

Now, since $\theta_{(j, e)}\left(C_{j}\right) \subset C_{\alpha(j, e)}, \operatorname{dim}\left(C_{\alpha(j, e)}\right) \geq \operatorname{dim}\left(\theta_{(j, e)}\left(C_{j}\right)\right)=\operatorname{dim}\left(C_{j}\right)$ for all $(j, e) \in \Gamma$. Let $1 \leq j \leq p$ and $1 \leq k \leq p$. By strong connectedness, there exists $\left[\left(j_{1}, e_{1}\right), \ldots,\left(j_{n}, e_{n}\right)\right]$ such that $j_{1}=j, \alpha\left(j_{i}, e_{i}\right)=j_{i+1}, 1 \leq i<n$ and $\alpha\left(j_{n}, e_{n}\right)=k$. So,

$\operatorname{dim}\left(C_{k}\right)=\operatorname{dim}\left(C_{\alpha\left(j_{n}, e_{n}\right)}\right) \geq \operatorname{dim}\left(C_{j_{n}}\right) \geq \operatorname{dim}\left(C_{j_{n-1}}\right) \geq \cdots \geq \operatorname{dim}\left(C_{j_{1}}\right)=\operatorname{dim}\left(C_{j}\right)$. Since $j$ and $k$ were arbitrary, it follows that $\operatorname{dim}\left(C_{j}\right)=\operatorname{dim}\left(C_{k}\right)$ for all $1 \leq j, k \leq$ $p$.

We introduce a 'weighted' Hausdorff measure using the strictly positive eigenvector $u_{\sigma}$ of $L_{\sigma}$ with eigenvalue $r\left(L_{\sigma}\right)$. Let $1 \leq j \leq p$. Define for $A_{j} \subset S_{j}$ and $\epsilon>0$

$\tilde{\mathcal{H}}_{\epsilon}^{\sigma}\left(A_{j}\right)=\inf \left\{\sum_{k=1}^{\infty}\left(u_{\sigma}\right)_{j}\left(\xi_{j k}\right)\left(\operatorname{diam}\left(A_{j k}\right)\right)^{\sigma}: A_{j} \subset \bigcup_{k=1}^{\infty} A_{j k}, \xi_{j k} \in A_{j k}, \operatorname{diam}\left(A_{j k}\right)<\epsilon\right\}$.

From Lemma 4.8, we know that there exist constants $0<l \leq L<\infty$ such that for $1 \leq j \leq p, l \leq\left(u_{\sigma}\right)_{j}(t) \leq L$ for all $t \in S_{j}$. This implies that, for $A_{j} \subset S_{j}$, $\tilde{\mathcal{H}}_{\epsilon}^{\sigma}\left(A_{j}\right)$ and $\mathcal{H}_{\epsilon}^{\sigma}\left(A_{j}\right)$ are equivalent:

$$
l \mathcal{H}_{\epsilon}^{\sigma}\left(A_{j}\right) \leq \tilde{\mathcal{H}}_{\epsilon}^{\sigma}\left(A_{j}\right) \leq L \mathcal{H}_{\epsilon}^{\sigma}\left(A_{j}\right) .
$$


Theorem 4.10. Assume that the hypotheses $\mathrm{H} 4.1, \mathrm{H} 4.2, \mathrm{H} 4.3$ and $\mathrm{H} 4.4$ are satisfied and let $C_{j} \subset S_{j}, 1 \leq j \leq p$, be the unique invariant list of compact, nonempty sets such that

$$
C_{i}=\bigcup_{(j, e) \in \Gamma_{i}} \theta_{(j, e)}\left(C_{j}\right) \text { for } 1 \leq i \leq p .
$$

If $\operatorname{dim}\left(C_{i}\right)$ denotes the Hausdorff dimension of $\left(C_{i}\right)$ and $\sigma_{0}$ denotes the unique nonnegative real number such that $r\left(L_{\sigma_{0}}\right)=1$, then $\operatorname{dim}\left(C_{i}\right) \leq \sigma_{0}$ for $1 \leq i \leq p$.

Proof. Fix $\epsilon>0$. Let $\delta>0$. We can choose a covering $\left\{A_{j k}\right\}_{k=1}^{\infty}$ of $C_{j}$ and points $\xi_{j k} \in A_{j k}$ such that $\operatorname{diam}\left(A_{j k}\right)<\epsilon$ and

$$
\sum_{k=1}^{\infty}\left(u_{\sigma}\right)_{j}\left(\xi_{j k}\right)\left(\operatorname{diam}\left(A_{j k}\right)\right)^{\sigma} \leq \tilde{\mathcal{H}}_{\epsilon}^{\sigma}\left(C_{j}\right)+\delta
$$

Since $C_{i}=\bigcup_{(j, e) \in \Gamma_{i}} \theta_{(j, e)}\left(C_{j}\right)$, we have that $\left\{\theta_{(j, e)}\left(A_{j k}\right): 1 \leq k<\infty,(j, e) \in \Gamma_{i}\right\}$ is a covering of $C_{i}$ with

$$
\operatorname{diam}\left(\theta_{(j, e)}\left(A_{j k}\right)\right) \leq c \operatorname{diam}\left(A_{j k}\right)<c \epsilon .
$$

Furthermore, using Lemma 4.3 and Remark 4.4, it is easy to see that there exists $\mu_{(j, e)}(\epsilon)>1$ with $\mu_{(j, e)}(\epsilon) \rightarrow 1$ as $\epsilon \rightarrow 0+$ such that

$$
\operatorname{diam}\left(\theta_{(j, e)}\left(A_{j k}\right)\right) \leq \mu_{(j, e)}(\epsilon)\left(D \theta_{(j, e)}\right)\left(\xi_{j k}\right) \operatorname{diam}\left(A_{j k}\right) .
$$

Let $\mu(\epsilon)=\max _{(j, e) \in \Gamma} \mu_{(j, e)}(\epsilon)$. Then

$$
\begin{aligned}
\tilde{\mathcal{H}}_{c \epsilon}^{\sigma}\left(C_{i}\right) & \leq \sum_{k=1}^{\infty} \sum_{(j, e) \in \Gamma_{i}}\left(u_{\sigma}\right)_{i}\left(\theta_{(j, e)}\left(\xi_{j k}\right)\right)\left(\operatorname{diam}\left(\theta_{(j, e)}\left(A_{j k}\right)\right)\right)^{\sigma} \\
& \leq(\mu(\epsilon))^{\sigma} \sum_{k=1}^{\infty} \sum_{(j, e) \in \Gamma_{i}}\left(u_{\sigma}\right)_{i}\left(\theta_{(j, e)}\left(\xi_{j k}\right)\right)\left(\left(D \theta_{(j, e)}\right)\left(\xi_{j k}\right)\right)^{\sigma}\left(\operatorname{diam}\left(A_{j k}\right)\right)^{\sigma} .
\end{aligned}
$$

Summing over $i, 1 \leq i \leq p$, we have

$$
\sum_{i=1}^{p} \tilde{\mathcal{H}}_{c \epsilon}^{\sigma}\left(C_{i}\right) \leq(\mu(\epsilon))^{\sigma} \sum_{i=1}^{p} \sum_{k=1}^{\infty} \sum_{(j, e) \in \Gamma_{i}}\left(u_{\sigma}\right)_{i}\left(\theta_{(j, e)}\left(\xi_{j k}\right)\right)\left(\left(D \theta_{(j, e)}\right)\left(\xi_{j k}\right)\right)^{\sigma}\left(\operatorname{diam}\left(A_{j k}\right)\right)^{\sigma} .
$$

Rearranging the sum, we get

$$
\begin{aligned}
\sum_{i=1}^{p} \tilde{\mathcal{H}}_{c \epsilon}^{\sigma}\left(C_{i}\right) & \leq(\mu(\epsilon))^{\sigma} \sum_{j=1}^{p} \sum_{k=1}^{\infty}\left(\operatorname{diam}\left(A_{j k}\right)\right)^{\sigma} \sum_{i:(j, e) \in \Gamma_{i}}\left(u_{\sigma}\right)_{i}\left(\theta_{(j, e)}\left(\xi_{j k}\right)\right)\left(\left(D \theta_{(j, e)}\right)\left(\xi_{j k}\right)\right)^{\sigma} \\
& =(\mu(\epsilon))^{\sigma} \sum_{j=1}^{p} \sum_{k=1}^{\infty}\left(L_{\sigma} u_{\sigma}\right)_{j}\left(\xi_{j k}\right)\left(\operatorname{diam}\left(A_{j k}\right)\right)^{\sigma} \\
& =(\mu(\epsilon))^{\sigma} r\left(L_{\sigma}\right) \sum_{j=1}^{p} \sum_{k=1}^{\infty}\left(u_{\sigma}\right)_{j}\left(\xi_{j k}\right)\left(\operatorname{diam}\left(A_{j k}\right)\right)^{\sigma}
\end{aligned}
$$

Thus, using (4.8), we get

$$
\sum_{i=1}^{p} \tilde{\mathcal{H}}_{c \epsilon}^{\sigma}\left(C_{i}\right) \leq(\mu(\epsilon))^{\sigma} r\left(L_{\sigma}\right) \sum_{j=1}^{p}\left(\tilde{\mathcal{H}}_{\epsilon}^{\sigma}\left(C_{j}\right)+\delta\right) .
$$


Since $c<1, \tilde{\mathcal{H}}_{\epsilon}^{\sigma}\left(C_{i}\right) \leq \tilde{\mathcal{H}}_{c \epsilon}^{\sigma}\left(C_{i}\right)$. Also $\delta>0$ was arbitrary. Therefore,

$$
\sum_{i=1}^{p} \tilde{\mathcal{H}}_{\epsilon}^{\sigma}\left(C_{i}\right) \leq(\mu(\epsilon))^{\sigma} r\left(L_{\sigma}\right) \sum_{i=1}^{p} \tilde{\mathcal{H}}_{\epsilon}^{\sigma}\left(C_{i}\right)
$$

Using Lemma 4.6. $r\left(L_{\sigma}\right)<1$ for all $\sigma>\sigma_{0}$. Since $\mu(\epsilon) \rightarrow 1$ as $\epsilon \rightarrow 0$, given $\sigma>\sigma_{0}$, we can choose $\epsilon>0$ small so that $(\mu(\epsilon))^{\sigma} r\left(L_{\sigma}\right)<1$. By the definition, $\tilde{\mathcal{H}}_{\epsilon}^{\sigma}\left(C_{i}\right)<\infty$ because we can take a finite $\epsilon$-cover of the compact set $C_{i}$. Thus, if $\sigma>\sigma_{0}$, (4.10) can hold only if

$$
\lim _{\epsilon \rightarrow 0+} \sum_{i=1}^{p} \tilde{\mathcal{H}}_{\epsilon}^{\sigma}\left(C_{i}\right)=0 .
$$

This implies that for each $i, 1 \leq i \leq p$ and $\sigma>\sigma_{0}, \lim _{\epsilon \rightarrow 0+} \tilde{\mathcal{H}}_{\epsilon}^{\sigma}\left(C_{i}\right)=0$, and hence using (4.7), $\lim _{\epsilon \rightarrow 0+} \mathcal{H}_{\epsilon}^{\sigma}\left(C_{i}\right)=0$, i.e., $\mathcal{H}^{\sigma}\left(C_{i}\right)=0$ for all $\sigma>\sigma_{0}$. Thus, by the definition of Hausdorff dimension, $\operatorname{dim}\left(C_{i}\right) \leq \sigma_{0}$.

We define for $0<\eta<\epsilon$, and $A_{j} \subset S_{j}$,

$$
\begin{aligned}
\tilde{\mathcal{H}}_{\epsilon, \eta}^{\sigma}\left(A_{j}\right):=\inf \left\{\sum_{k=1}^{\infty}\left(u_{\sigma}\right)_{j}\left(\xi_{j k}\right)\left(\operatorname{diam}\left(A_{j k}\right)\right)^{\sigma}\right. \\
\left.\quad: A_{j} \subset \bigcup_{k=1}^{\infty} A_{j k}, \xi_{j k} \in A_{j k}, \eta<\operatorname{diam}\left(A_{j k}\right)<\epsilon\right\} .
\end{aligned}
$$

The quantity $\tilde{\mathcal{H}}_{\epsilon, \eta}^{\sigma}\left(A_{j}\right)$ will be technically useful later, primarily because it is strictly positive whenever it is defined. However, caution is necessary in using this tool. It is easy to see that $\tilde{\mathcal{H}}^{\sigma}\left(A_{j}\right)$ and $\tilde{\mathcal{H}}_{\epsilon}^{\sigma}\left(A_{j}\right)$ depend only on the metric space $\left(A_{j}, d_{j}\right)$. In contrast, $\tilde{\mathcal{H}}_{\epsilon, \eta}^{\sigma}\left(A_{j}\right)$ depends also on $S_{j}$. If $A_{j} \subset T_{j} \subset S_{j}$ one could give an analogous definition in which one only allows sets $A_{j k} \subset T_{j}$ :

$$
\begin{aligned}
& \tilde{\mathcal{H}}_{\epsilon, \eta}^{\sigma}\left(A_{j} ; T_{j}\right):=\inf \left\{\sum_{k=1}^{\infty}\left(u_{\sigma}\right)_{j}\left(\xi_{j k}\right)\left(\operatorname{diam}\left(A_{j k}\right)\right)^{\sigma}\right. \\
&\left.\quad: A_{j} \subset \bigcup_{k=1}^{\infty} A_{j k} \subset T_{j}, \xi_{j k} \in A_{j k}, \eta<\operatorname{diam}\left(A_{j k}\right)<\epsilon\right\} .
\end{aligned}
$$

In this cumbersome notation, $\tilde{\mathcal{H}}_{\epsilon}^{\sigma}\left(A_{j}\right)=\tilde{\mathcal{H}}_{\epsilon, \eta}^{\sigma}\left(A_{j} ; S_{j}\right)$ for $A_{j} \subset S_{j}$. If $A_{j}$ is compact and $A_{j} \subset T_{j} \subset S_{j}$, it may happen that $\tilde{\mathcal{H}}_{\epsilon, \eta}^{\sigma}\left(A_{j} ; T_{j}\right)$ and $\tilde{\mathcal{H}}_{\epsilon, \eta}^{\sigma}\left(A_{j} ; S_{j}\right)$ are both defined but are unequal. For the unique list of nonempty compact sets $C_{j} \subset S_{j}$, $1 \leq j \leq p$, ensured by Theorem 2.3 and for $A_{j} \subset C_{j}$, we shall, in our later work, sometimes consider $\tilde{\mathcal{H}}_{\epsilon, \eta}^{\sigma}\left(A_{j} ; C_{j}\right)$ rather than $\tilde{\mathcal{H}}_{\epsilon, \eta}^{\sigma}\left(A_{j} ; S_{j}\right)$.

In general, if $A_{j} \subset T_{j} \subset S_{j}$ and $A_{j}$ is compact and $0<\eta<\epsilon$, in order that $\tilde{\mathcal{H}}_{\epsilon, \eta}^{\sigma}\left(A_{j} ; T_{j}\right)$ be defined and finite, it is necessary and sufficient that there exist sets $A_{j k} \subset T_{j}, 1 \leq k \leq m<\infty$, with $\eta<\operatorname{diam}\left(A_{j k}\right)<\epsilon$ for $1 \leq k \leq m$ and $A_{j} \subset \bigcup_{k=1}^{m} A_{j k}$. The existence or nonexistence of such sets may be a delicate question. If $A_{j}$ contains an isolated point of $T_{j}$, such sets $A_{j k}$ do not exist for all small $\epsilon$ and all $\eta$ with $0<\eta<\epsilon$. If $T_{j}$ is a complete perfect metric space, $A_{j} \subset T_{j}$ is compact and $\epsilon>0, \tilde{\mathcal{H}}_{\epsilon, \eta}^{\sigma}\left(A_{j} ; T_{j}\right)$ will be defined for all sufficiently small $\eta>0$. To see this, use compactness of $A_{j}$ to find finitely many open balls $B_{k} \subset T_{j}$, $1 \leq k \leq n$, with radius $r<\epsilon / 2$ and centers in $A_{j}$, such that $A_{j} \subset \bigcup_{k=1}^{n} B_{k}$. Since 
$T_{j}$ is perfect, each $B_{k}$ contains an accumulation point, so $\operatorname{diam}\left(B_{k}\right)>\eta_{k}>0$ and $\operatorname{diam}\left(B_{k}\right)>\eta:=\min \left\{\eta_{k}: 1 \leq k \leq n\right\}$ and $\operatorname{diam}\left(B_{k}\right) \leq 2 r<\epsilon$.

Lemma 4.11. Assume that $\mathrm{H} 4.1, \mathrm{H} 4.2, \mathrm{H} 4.3$ and $\mathrm{H} 4.4$ are satisfied. Let $1 \leq j \leq p$ and let $A_{j}$ be a compact subset of $S_{j}$. If $\sigma \geq 0$ and $\epsilon>0$, then

$$
\lim _{\eta \rightarrow 0+} \tilde{\mathcal{H}}_{\epsilon, \eta}^{\sigma}\left(A_{j}\right)=\tilde{\mathcal{H}}_{\epsilon}^{\sigma}\left(A_{j}\right) \text {. }
$$

Proof. For $0<\eta<\epsilon$, we have $\tilde{\mathcal{H}}_{\epsilon, \eta}^{\sigma}\left(A_{j}\right) \geq \tilde{\mathcal{H}}_{\epsilon}^{\sigma}\left(A_{j}\right)$ because the infimum is taken over a smaller set. So,

$$
\lim _{\eta \rightarrow 0+} \tilde{\mathcal{H}}_{\epsilon, \eta}^{\sigma}\left(A_{j}\right) \geq \tilde{\mathcal{H}}_{\epsilon}^{\sigma}\left(A_{j}\right) .
$$

To prove the reverse inequality, take $\delta>0$ and choose a covering $\left\{A_{j k}: 1 \leq k<\infty\right\}$ of $A_{j}$ by sets $A_{j k}$ with $\operatorname{diam}\left(A_{j k}\right)<\epsilon, 1 \leq k<\infty$ such that

$$
\inf \left\{\sum_{k=1}^{\infty}\left(u_{\sigma}\right)_{j}\left(\xi_{j k}\right)\left(\operatorname{diam}\left(A_{j k}\right)\right)^{\sigma}: \xi_{j k} \in A_{j k}\right\} \leq \tilde{\mathcal{H}}_{\epsilon}^{\sigma}\left(A_{j}\right)+\delta .
$$

Without loss of generality, we can assume that the sets $A_{j k}, k \geq 1$ are open. Since $A_{j}$ is compact, there exists a finite open subcover of $A_{j}$, so there exists an integer $l<\infty$ such that

$$
A_{j} \subset \bigcup_{k=1}^{l} A_{j k} .
$$

Let $0<\eta_{0}<\epsilon$ be such that $\eta_{0}<\min _{1 \leq k \leq l} \operatorname{diam}\left(A_{j k}\right)$. Then, for $0<\eta<\eta_{0}$, we have

$$
\tilde{\mathcal{H}}_{\epsilon, \eta}^{\sigma}\left(A_{j}\right) \leq \inf \left\{\sum_{k=1}^{l}\left(u_{\sigma}\right)_{j}\left(\xi_{j k}\right)\left(\operatorname{diam}\left(A_{j k}\right)\right)^{\sigma}: \xi_{j k} \in A_{j k}\right\} .
$$

So, for every $\delta>0$, there exists $\eta_{0}, 0<\eta_{0}<\epsilon$, such that

$$
\tilde{\mathcal{H}}_{\epsilon, \eta}^{\sigma}\left(A_{j}\right) \leq \tilde{\mathcal{H}}_{\epsilon}^{\sigma}\left(A_{j}\right)+\delta \quad \text { for } 0<\eta<\eta_{0} .
$$

This shows

$$
\lim _{\eta \rightarrow 0+} \tilde{\mathcal{H}}_{\epsilon, \eta}^{\sigma}\left(A_{j}\right) \leq \tilde{\mathcal{H}}_{\epsilon}^{\sigma}\left(A_{j}\right)
$$

and completes the proof of the lemma.

Lemma 4.12. Assume that $\mathrm{H} 4.1, \mathrm{H} 4.2, \mathrm{H} 4.3$ and $\mathrm{H} 4.4$ are satisfied. Let $1 \leq j \leq p$ and let $A_{j}$ be a compact subset of $S_{j}$. Let $\sigma>0$ be such that $\mathcal{H}^{\sigma}\left(A_{j}\right)=0$. Then for every $\epsilon_{1}$ and $\epsilon_{2}$ with $0<\epsilon_{1}<\epsilon_{2}$, there exists an $\eta_{0}>0$ such that for any $B_{j} \subset A_{j}$,

$$
\tilde{\mathcal{H}}_{\epsilon_{1}, \eta}^{\sigma}\left(B_{j}\right)=\tilde{\mathcal{H}}_{\epsilon_{2}, \eta}^{\sigma}\left(B_{j}\right) \quad \text { for } 0<\eta<\eta_{0} .
$$

Proof. Since $\mathcal{H}^{\sigma}\left(A_{j}\right)=0$, it follows that $\mathcal{H}_{\epsilon}^{\sigma}\left(A_{j}\right)=0$ for every $\epsilon>0$. By using (4.7), it also follows that $\tilde{\mathcal{H}}_{\epsilon}^{\sigma}\left(A_{j}\right)=0$ for every $\epsilon>0$. So, by Lemma 4.11.

$$
\lim _{\eta \rightarrow 0+} \tilde{\mathcal{H}}_{\epsilon_{2}, \eta}^{\sigma}\left(A_{j}\right)=0 .
$$

This implies that there exists $\eta_{0}>0$ such that for $0<\eta<\eta_{0}$,

$$
\tilde{\mathcal{H}}_{\epsilon_{2}, \eta}^{\sigma}\left(A_{j}\right)<l \epsilon_{1}{ }^{\sigma},
$$

where, as before, $l>0$ is such that $\left(u_{\sigma}\right)_{j}(t) \geq l$ for all $t \in S_{j}$. 
If $B_{j} \subset A_{j}$, then $\tilde{\mathcal{H}}_{\epsilon_{2}, \eta}^{\sigma}\left(B_{j}\right) \leq \tilde{\mathcal{H}}_{\epsilon_{2}, \eta}^{\sigma}\left(A_{j}\right)<l \epsilon_{1}{ }^{\sigma}$. Therefore, given $\delta>0$, there exists a covering $\left\{B_{j k}: k \geq 1\right\}$ of $B_{j}$ such that $\eta<\operatorname{diam}\left(B_{j k}\right)<\epsilon_{2}$ for $k \geq 1$ and

$$
\inf \left\{\sum_{k=1}^{\infty}\left(u_{\sigma}\right)_{j}\left(\xi_{j k}\right)\left(\operatorname{diam}\left(B_{j k}\right)\right)^{\sigma}: \xi_{j k} \in B_{j k}\right\} \leq \tilde{\mathcal{H}}_{\epsilon_{2}, \eta}^{\sigma}\left(B_{j}\right)+\delta<l \epsilon_{1} \sigma .
$$

Next we claim that actually $\operatorname{diam}\left(B_{j k}\right)<\epsilon_{1}$ for all $k \geq 1$. Suppose not. Then there exists an index $k_{1}$ such that $\operatorname{diam}\left(B_{j k_{1}}\right) \geq \epsilon_{1}$. By considering the term corresponding to index $k_{1}$ in the sum and using $\left(u_{\sigma}\right)_{j}\left(\xi_{j k}\right) \geq l$, we get

$$
\inf \left\{\sum_{k=1}^{\infty}\left(u_{\sigma}\right)_{j}\left(\xi_{j k}\right)\left(\operatorname{diam}\left(B_{j k}\right)\right)^{\sigma}: \xi_{j k} \in B_{j k}\right\} \geq l \epsilon_{1}{ }^{\sigma},
$$

which gives a contradiction. Thus $\operatorname{diam}\left(B_{j k}\right)<\epsilon_{1}$ for all $k \geq 1$ and we conclude that

$$
\tilde{\mathcal{H}}_{\epsilon_{1}, \eta}^{\sigma}\left(B_{j}\right) \leq \tilde{\mathcal{H}}_{\epsilon_{2}, \eta}^{\sigma}\left(B_{j}\right)+\delta \text { for } 0<\eta<\eta_{0} .
$$

Since $\delta>0$ was arbitrary, we conclude

$$
\tilde{\mathcal{H}}_{\epsilon_{1}, \eta}^{\sigma}\left(B_{j}\right) \leq \tilde{\mathcal{H}}_{\epsilon_{2}, \eta}^{\sigma}\left(B_{j}\right) \quad \text { for } 0<\eta<\eta_{0} .
$$

Since $\tilde{\mathcal{H}}_{\epsilon, \eta}^{\sigma}\left(B_{j}\right)$ is a decreasing function of $\epsilon$, the reverse inequality is obvious. Thus, we obtain

$$
\tilde{\mathcal{H}}_{\epsilon_{1}, \eta}^{\sigma}\left(B_{j}\right)=\tilde{\mathcal{H}}_{\epsilon_{2}, \eta}^{\sigma}\left(B_{j}\right) \quad \text { for } 0<\eta<\eta_{0} .
$$

If $C_{j} \subset S_{j}, 1 \leq j \leq p$, is the unique invariant list guaranteed by Theorem 2.3. it is convenient in the arguments below to work in the compact sets $C_{j}$ rather than $S_{j}$. For this to be permissible, we must first show that each set $C_{j}$ is a perfect metric space. Our first lemma in this direction has essentially been established in the proof of Lemma 4.9 and is left to the reader.

Lemma 4.13. Assume hypotheses $\mathrm{H} 4.1, \mathrm{H} 4.2, \mathrm{H} 4.3$ and $\mathrm{H} 4.4$ and let $C_{j} \subset S_{j}$, $1 \leq j \leq p$, be the invariant sets guaranteed by Theorem 2.3. Then there exist $m_{0}>0$ and $\delta_{0}>0$ such that for all $(j, e) \in \Gamma$ and for all $s, t \in S_{j}$ with $s \in C_{j}$ and $d_{j}(s, t)<\delta_{0}$,

$$
d_{\alpha(j, e)}\left(\theta_{(j, e)}(s), \theta_{(j, e)}(t)\right) \geq m_{0} d_{j}(s, t) .
$$

Lemma 4.14. Assume hypotheses $\mathrm{H} 4.1, \mathrm{H} 4.2, \mathrm{H} 4.3$ and $\mathrm{H} 4.4$ and let $C_{j} \subset S_{j}$, $1 \leq j \leq p$, be the invariant sets guaranteed by Theorem 2.3. Then either (a) each $C_{j}, 1 \leq j \leq p$, is a perfect metric space or (b) each $C_{j}, 1 \leq j \leq p$, is a finite set. If $\left.\theta_{(j, e)}\right|_{C_{j}}$ is one-to-one for all $(j, e) \in \Gamma$ and each $C_{j}$ is a finite set, then each $C_{j}$ contains exactly one element for $1 \leq j \leq p$.

Proof. Assume that $C_{i}$ is not a finite set for some $i, 1 \leq i \leq p$. It follows that there exists $\tau_{i} \in C_{i}$ such that $\tau_{i}$ is an accumulation point of $C_{i}$. If $1 \leq k \leq p$, then H4.4 implies that there exist $\left(j_{l}, e_{l}\right) \in \Gamma, 1 \leq l \leq n$, with $\alpha\left(j_{l}, e_{l}\right)=j_{l+1}$ for $1 \leq l<n, j_{1}=i$ and $\alpha\left(j_{n}, e_{n}\right)=k$. Writing $(J, E)=\left[\left(j_{1}, e_{1}\right),\left(j_{2}, e_{2}\right), \ldots,\left(j_{n}, e_{n}\right)\right]$, $\theta_{(J, E)}\left(\tau_{i}\right) \in C_{k}$. Also, because each $\theta_{(j, e)}$ is a contraction, Lemma 4.13 implies that $\theta_{(J, E)}$ is one-to-one on $B_{r_{0}}\left(\tau_{i}\right):=\left\{t \in C_{i}: d_{i}\left(t, \tau_{i}\right)<r_{0}\right\}$, where $r_{0}=\delta_{0} / 2$ and $\delta_{0}$ is as in Lemma 4.13. Using this fact, we see that $\theta_{(J, E)}\left(\tau_{i}\right):=\tau_{k}$ is an accumulation point of $C_{k}$.

If $V=\{1,2, \ldots, p\}, \Theta$ is as in the proof of Theorem 2.3 and $A_{j}$ is any closed, bounded, nonempty subset of $S_{j}$ for $1 \leq j \leq p$, then, because $\Theta$ is a contraction map 
in the Hausdorff metric, $\Theta^{n}\left(\left(A_{1}, A_{2}, \ldots, A_{p}\right)\right)$ converges in the Hausdorff metric to $\left(C_{1}, C_{2}, \ldots, C_{p}\right)$ as $n \rightarrow \infty$. We apply this result to $A_{j}=\left\{\tau_{j}\right\}$, where $\tau_{j}$ is an accumulation point of $C_{j}$ for $1 \leq j \leq p$. If $k, 1 \leq k \leq p$, and $\sigma \in C_{k}$ are fixed, it follows that for each $n \geq 1$, there exists $\left(J^{n}, E^{n}\right)=\left[\left(j_{1}^{n}, e_{1}^{n}\right),\left(j_{2}^{n}, e_{2}^{n}\right) \ldots,\left(j_{n}^{n}, e_{n}^{n}\right)\right]$ with $\left(j_{i}^{n}, e_{i}^{n}\right) \in \Gamma$ for $1 \leq i \leq n, \alpha\left(j_{i}^{n}, e_{i}^{n}\right)=\left(j_{i+1}^{n}, e_{i+1}^{n}\right)$ for $1 \leq i<n, \alpha\left(j_{n}^{n}, e_{n}^{n}\right)=k$ and $\theta_{\left(J^{n}, E^{n}\right)}\left(\tau_{j_{1}^{n}}\right) \rightarrow \sigma$ as $n \rightarrow \infty$. For notational convenience, write $i_{n}:=j_{1}^{n}$. If $r_{0}$ and $\delta_{0}$ are as above and $V_{n}=\left\{t \in C_{i_{n}}: d\left(t, \tau_{i_{n}}\right)<r_{0}\right\},\left.\theta_{\left(J^{n}, E^{n}\right)}\right|_{V_{n}}$ is one-to-one and $\operatorname{diam}\left(\theta_{\left(J^{n}, E^{n}\right)}\left(V_{n}\right)\right) \leq \delta_{0} c^{n}$, where $\operatorname{Lip}\left(\theta_{(j, e)}\right) \leq c<1$ for all $(j, e) \in \Gamma$. Because we also know that $\tau_{i_{n}}$ is an accumulation point of $C_{i_{n}}$, it follows that there exist points $s_{n}, t_{n} \in \theta_{\left(J^{n}, E^{n}\right)}\left(V_{n}\right), s_{n} \neq t_{n}$ and necessarily $\lim _{n \rightarrow \infty} s_{n}=\lim _{n \rightarrow \infty} t_{n}=\sigma$. This, in turn, implies that $\sigma$ is an accumulation point of $C_{k}$ and that $C_{k}$ is a perfect metric space for $1 \leq k \leq p$.

If $\left.\theta_{(j, e)}\right|_{C_{j}}$ is one-to-one for each $(j, e) \in \Gamma$ and if each $C_{j}$ is a finite set, we claim that each $C_{j}$ is a one-point set. If not, then there exists $j, 1 \leq j \leq p$, such that $C_{j}$ contains at least two points. Using $\mathrm{H} 4.4$ and the fact that each $\left.\theta_{(i, e)}\right|_{C_{i}}$ is one-to-one, it follows that $C_{k}$ contains at least two points for $1 \leq k \leq p$. Define $\rho=\inf \left\{d_{j}(s, t): s, t \in C_{j}, s \neq t, 1 \leq j \leq p\right\}$, so $\rho>0$, and select $j$ and $s, t \in C_{j}$ with $d_{j}(s, t)=\rho$. Because $\theta_{(j, e)}$ is a contraction and is one-to-one on $C_{j}$, $\theta_{(j, e)}(s) \neq \theta_{(j, e)}(t)$ and $d_{\alpha(j, e)}\left(\theta_{(j, e)}(s), \theta_{(j, e)}(t)\right)<\rho$, a contradiction.

Assume H4.1, H4.2, H4.3 and H4.4. If $f=\left(f_{1}, f_{2}, \ldots, f_{p}\right) \in \prod_{j=1}^{p} C\left(C_{j}\right)$, where $\left(C_{1}, C_{2}, \ldots, C_{p}\right)$ is as in Theorem 2.3, we can define $\Lambda_{\sigma}: \prod_{j=1}^{p} C\left(C_{j}\right) \rightarrow$ $\prod_{j=1}^{p} C\left(C_{j}\right)$ by (4.4). Furthermore, if each $C_{j}$ is a perfect metric space (compare Lemma 4.14) and $\psi_{(j, e)}: C_{j} \rightarrow C_{\alpha(j, e)}$ is defined by $\psi_{(j, e)}(s)=\theta_{(j, e)}(s)$ for $s \in C_{j}$, then $D \psi_{(j, e)}(s)$ is defined for all $s \in C_{j}$ and $D \psi_{(j, e)}(s)=D \theta_{(j, e)}(s)$ for $s \in C_{j}$. In any event, by using Lemma 4.8, it follows easily that the spectral radius of $L_{\sigma}$ equals the spectral radius of $\Lambda_{\sigma}$ and is the eigenvalue of the strictly positive eigenvector $u_{\sigma}$ of Lemma 4.8. The corresponding positive eigenvector $v_{\sigma}$ for $\Lambda_{\sigma}$ is given by $\left(v_{\sigma}\right)_{j}(s)=\left(u_{\sigma}\right)_{j}(s)$ for $s \in C_{j}$. If each $C_{j}$ is a finite set, $\prod_{j=1}^{p} C\left(C_{j}\right)$ is finite dimensional and determining $r\left(\Lambda_{\sigma}\right)=r\left(L_{\sigma}\right)$ is a finite dimensional problem.

Lemma 4.15. Assume that hypotheses $\mathrm{H} 4.1, \mathrm{H} 4.2, \mathrm{H} 4.3$ and $\mathrm{H} 4.4$ are satisfied and let $C_{j} \subset S_{j}, 1 \leq j \leq p$ be the unique invariant list such that

$$
C_{i}=\bigcup_{(j, e) \in \Gamma_{i}} \theta_{(j, e)}\left(C_{j}\right) \text { for } 1 \leq i \leq p .
$$

Also assume that the map $\left.\theta_{(j, e)}\right|_{C_{j}}$ is one-to-one for all $(j, e) \in \Gamma$ and that, for some $j, C_{j}$ has more than one element. Suppose that for all $(j, e) \in \Gamma$ and $\left(j^{\prime}, e^{\prime}\right) \in \Gamma$ such that $(j, e) \neq\left(j^{\prime}, e^{\prime}\right)$ and $\alpha(j, e)=\alpha\left(j^{\prime}, e\right)$, we have that $\theta_{(j, e)}\left(C_{j}\right) \cap \theta_{\left(j^{\prime}, e^{\prime}\right)}\left(C_{j^{\prime}}\right)=\emptyset$. Finally, suppose, for $\nu>0$, that $\left\{\Lambda_{\beta}^{i}: \beta \in \mathcal{A}_{i}(\nu)\right\}$ is a partition of $C_{i}$ consisting of compact subsets $\Lambda_{\beta}^{i}$ of $C_{i}$ with $\operatorname{diam}\left(\Lambda_{\beta}^{i}\right)<\nu$. Then there exist $\epsilon_{0}>0$ and $\nu_{0}>0$ such that for $0<\epsilon<\epsilon_{0}, 0<\nu<\nu_{0}, 0<\eta<m \epsilon$ and $1 \leq i \leq p$,

$$
\mu(\nu) r\left(L_{\sigma}\right) \tilde{\mathcal{H}}_{\mu_{1}(\nu) \epsilon, \eta}^{\sigma}\left(\Lambda_{\beta}^{i} ; C_{i}\right) \leq \sum_{e \in E_{i}} \tilde{\mathcal{H}}_{m \epsilon, \eta}^{\sigma}\left(\theta_{(i, e)}\left(\Lambda_{\beta}^{i}\right) ; C_{\alpha(i, e)}\right),
$$

where $\mu(\nu) \rightarrow 1$ and $\mu_{1}(\nu) \rightarrow 1$ as $\nu \rightarrow 0+$ and $\left(D \theta_{(i, e)}\right)(t)>m$ for all $t \in C_{i}$ and all $(i, e) \in \Gamma$. 
Remark. For compact sets $B \subset C_{k}$, we shall always use $\tilde{\mathcal{H}}_{\epsilon_{1}, \eta_{1}}^{\sigma}\left(B ; C_{k}\right)$ in the following proof, so for notational simplicity we shall write $\tilde{\mathcal{H}}_{\epsilon_{1}, \eta_{1}}^{\sigma}(B)$ instead of $\tilde{\mathcal{H}}_{\epsilon_{1}, \eta_{1}}^{\sigma}\left(B ; C_{k}\right)$.

We interpret (4.12) as meaning that $\eta$ with $0<\eta<m \epsilon$ is such that $\tilde{\mathcal{H}}_{m \epsilon, \eta}^{\sigma}\left(\theta_{(i, e)}\left(\Lambda_{\beta}^{i}\right)\right)$ is defined and finite for all $e \in E_{i}$ and that this implies that for $\epsilon^{*}:=\mu_{1}(\nu) \epsilon, \tilde{\mathcal{H}}_{\epsilon^{*}, \eta}^{\sigma}\left(\Lambda_{\beta}^{i}\right)$ is defined and finite and (4.12) is satisfied.

Proof. Lemma 4.14 implies that each $C_{j}, 1 \leq j \leq p$, is a compact, perfect metric space. Since we assume that $\theta_{(j, e)}\left(C_{j}\right) \cap \theta_{\left(j^{\prime}, e^{\prime}\right)}\left(C_{j^{\prime}}\right)=\emptyset$ whenever $(j, e) \neq\left(j^{\prime}, e^{\prime}\right)$ and $\alpha(j, e)=\alpha\left(j^{\prime}, e^{\prime}\right)$, we can select $\epsilon_{0}$ so that $d_{k}(s, t)>\epsilon_{0}$ whenever $(j, e) \neq$ $\left(j^{\prime}, e^{\prime}\right) \in \Gamma, \alpha(j, e)=\alpha\left(j^{\prime}, e^{\prime}\right)=k, s \in \theta_{(j, e)}\left(C_{j}\right)$ and $t \in \theta_{\left(j^{\prime}, e^{\prime}\right)}\left(C_{j^{\prime}}\right)$. Since, for $1 \leq k \leq p, C_{k}=\bigcup_{\substack{(j, e) \in \Gamma \\ \alpha(j, e)=k}} \theta_{(j, e)}\left(C_{j}\right)$, it follows that, as a subset of the metric space $\left(C_{k}, d_{k}\right), \theta_{(j, e)}\left(C_{j}\right)$ is both compact and open for all $(j, e) \in \Gamma$ with $\alpha(j, e)=k$. Furthermore, denoting by $N_{\delta}(B)$ the closed $\delta$-neighborhood in $C_{k}$ of a compact set $B \subset C_{k}, N_{\delta}\left(\theta_{(j, e)}\left(C_{j}\right)\right)=\theta_{(j, e)}\left(C_{j}\right)$ for all $(j, e) \in \Gamma$ with $\alpha(j, e)=k$ and all $\delta$ with $0<\delta \leq \epsilon_{0}$.

Fix $\nu>0,1 \leq i \leq p, \Lambda_{\beta}^{i}$ with $\beta \in \mathcal{A}_{i}(\nu)$. Suppose that $\epsilon_{0}$ is as above. By decreasing $\epsilon_{0}$ further we can also assume that $\epsilon_{0} \leq(1-c) \nu /(2 m)$. Suppose that $0<\epsilon \leq \epsilon_{0}, 0<\eta<m \epsilon$ and that $\tilde{\mathcal{H}}_{m \epsilon, \eta}^{\sigma}\left(\theta_{(i, e)}\left(\Lambda_{\beta}^{i}\right)\right)$ is defined and finite for all $e \in E_{i}$. For any $\delta>0$, there exists an open (in the relative topology of $C_{\alpha(i, e)}$ ), necessarily finite covering of $\theta_{(i, e)}\left(\Lambda_{\beta}^{i}\right),\left\{A_{j}: 1 \leq j \leq k\right\}$, in $C_{\alpha(i, e)}$, such that $\eta<\operatorname{diam}\left(A_{j}\right)<m \epsilon$ for $1 \leq j \leq k$ and

$$
\inf \left\{\sum_{j=1}^{k}\left(u_{\sigma}\right)_{\alpha(i, e)}\left(\xi_{j}\right)\left(\operatorname{diam}\left(A_{j}\right)\right)^{\sigma}: \xi_{j} \in A_{j}\right\} \leq \tilde{\mathcal{H}}_{m \epsilon, \eta}^{\sigma}\left(\theta_{(i, e)}\left(\Lambda_{\beta}^{i}\right)\right)+\delta .
$$

We denote by $N_{m \epsilon}\left(\theta_{(i, e)}\left(\Lambda_{\beta}^{i}\right)\right)$ the closed $m \epsilon$-neighborhood of $\theta_{(i, e)}\left(\Lambda_{\beta}^{i}\right)$ in $C_{\alpha(i, e)}$, and because $m \epsilon \leq \epsilon_{0}$, we observe that $N_{m \epsilon}\left(\theta_{(i, e)}\left(\Lambda_{\beta}^{i}\right)\right) \subset \theta_{(i, e)}\left(C_{i}\right)$. We have that $\operatorname{diam}\left(A_{j}\right)<m \epsilon$, and we can assume that $A_{j}$ intersects $\theta_{(i, e)}\left(\Lambda_{\beta}^{i}\right)$ for $1 \leq j \leq k$, so

$$
\theta_{(i, e)}\left(\Lambda_{\beta}^{i}\right) \subset \bigcup_{j=1}^{k} A_{j} \subset N_{m \epsilon}\left(\theta_{(i, e)}\left(\Lambda_{\beta}^{i}\right)\right) \subset \theta_{(i, e)}\left(C_{i}\right) .
$$

Since $\operatorname{diam}\left(\theta_{(i, e)}\left(\Lambda_{\beta}^{i}\right)\right) \leq c \operatorname{diam}\left(\Lambda_{\beta}^{i}\right)<c \nu$,

$$
\operatorname{diam}\left(N_{m \epsilon}\left(\theta_{(i, e)}\left(\Lambda_{\beta}^{i}\right)\right)\right) \leq c \nu+2 m \epsilon<\nu
$$

since we assume that $\epsilon<\epsilon_{0} \leq(1-c) \nu /(2 m)$. Since $\left.\theta_{(i, e)}\right|_{C_{i}}$ is one-to-one, we derive from (4.14) that we have $\theta_{(i, e)}^{-1}: \theta_{(i, e)}\left(C_{i}\right) \rightarrow C_{i}$ and

$$
\Lambda_{\beta}^{i} \subset \bigcup_{j=1}^{k} \theta_{(i, e)}^{-1}\left(A_{j}\right) \subset C_{i} .
$$

Using Lemma 4.3 and Remark 4.4, there exists $\mu_{1}(\nu)$ with $\mu_{1}(\nu) \rightarrow 1+$ as $\nu \rightarrow 0+$ and

$$
\mu_{1}(\nu)^{-1}\left(D \theta_{(i, e)}^{-1}\right)(y) \leq \frac{d\left(\theta_{(i, e)}^{-1}(x), \theta_{(i, e)}^{-1}(y)\right)}{d(x, y)} \leq \mu_{1}(\nu)\left(D \theta_{(i, e)}^{-1}\right)(y)
$$


for all $x, y \in \theta_{(i, e)}\left(C_{i}\right)$ with $0<d(x, y)<\nu$ and, in particular, for all $x, y \in$ $N_{m \epsilon}\left(\theta_{(i, e)}\left(\Lambda_{\beta}^{i}\right)\right)$. Note that $\mu_{1}(\nu)$ can be chosen to be independent of $(i, e) \in \Gamma$. We write $d$ instead of $d_{i}$ or $d_{\alpha(i, e)}$ here and below. In particular, for any $x, y \in A_{j}$, $1 \leq j \leq k$

$d\left(\theta_{(i, e)}^{-1}(x), \theta_{(i, e)}^{-1}(y)\right) \leq \mu_{1}(\nu)\left(D \theta_{(i, e)}^{-1}\right)(y) d(x, y) \leq \mu_{1}(\nu)\left(\frac{1}{\left(D \theta_{(i, e)}\right)\left(\theta_{(i, e)}^{-1} y\right)}\right) \operatorname{diam}\left(A_{j}\right)$.

Using the compactness of $\theta_{(i, e)}^{-1}\left(N_{m \epsilon}\left(\theta_{(i, e)}\left(\Lambda_{\beta}^{i}\right)\right)\right)$, we see that there exists $\tau_{1} \in$ $\theta_{(i, e)}^{-1}\left(N_{m \epsilon}\left(\theta_{(i, e)}\left(\Lambda_{\beta}^{i}\right)\right)\right)$ with

$$
\operatorname{diam}\left(\theta_{(i, e)}^{-1}\left(A_{j}\right)\right) \leq \mu_{1}(\nu) \frac{1}{\left(D \theta_{(i, e)}\right)\left(\tau_{1}\right)} \operatorname{diam}\left(A_{j}\right) .
$$

Since $\operatorname{diam}\left(A_{j}\right)<m \epsilon$, (4.18) implies

$$
\operatorname{diam}\left(\theta_{(i, e)}^{-1}\left(A_{j}\right)\right)<\mu_{1}(\nu)\left(\frac{1}{m}\right) m \epsilon=\mu_{1}(\nu) \epsilon
$$

By choosing $x_{0}, y_{0} \in N_{m \epsilon}\left(\theta_{(i, e)}\left(\Lambda_{\beta}^{i}\right)\right)$ with $d\left(x_{0}, y_{0}\right)=\operatorname{diam}\left(A_{j}\right)$, we also obtain

$$
\operatorname{diam}\left(\theta_{(i, e)}^{-1}\left(A_{j}\right)\right) \geq d\left(\theta_{(i, e)}^{-1}\left(x_{0}\right), \theta_{(i, e)}^{-1}\left(y_{0}\right)\right) \geq \mu_{1}(\nu)^{-1}\left(\frac{1}{\left(D \theta_{(i, e)}\right)\left(\theta_{(i, e)}^{-1} y_{0}\right)}\right) \operatorname{diam}\left(A_{j}\right) .
$$

Since $\operatorname{diam}\left(A_{j}\right)>\eta$ and $\left(D \theta_{(i, e)}\right)\left(\theta_{(i, e)}^{-1} y_{0}\right) \leq c<1$, we find that $\operatorname{diam}\left(\theta_{(i, e)}^{-1}\left(A_{j}\right)\right) \geq$ $\mu_{1}(\nu)^{-1} c^{-1} \eta$. It follows that, assuming we originally chose $\nu_{0}$ such that $\mu_{1}(\nu) c<1$ for $0<\nu<\nu_{0}, \operatorname{diam}\left(\theta_{(i, e)}^{-1}\left(A_{j}\right)\right)>\eta$. Since $\Lambda_{\beta}^{i} \subset \bigcup_{j=1}^{k} \theta_{(i, e)}^{-1}\left(A_{j}\right)$, we conclude that

$$
\tilde{\mathcal{H}}_{\mu_{1}(\nu) \epsilon, \eta}^{\sigma}\left(\Lambda_{\beta}^{i}\right) \leq \inf \left\{\sum_{j=1}^{k}\left(u_{\sigma}\right)_{i}\left(\zeta_{j}\right)\left(\operatorname{diam}\left(\theta_{(i, e)}^{-1}\left(A_{j}\right)\right)\right)^{\sigma}: \zeta_{j} \in \theta_{(i, e)}^{-1}\left(A_{j}\right)\right\} .
$$

Using (4.18) and writing $\epsilon^{*}=\mu_{1}(\nu) \epsilon$,

$$
\begin{array}{r}
\tilde{\mathcal{H}}_{\epsilon^{*}, \eta}^{\sigma}\left(\Lambda_{\beta}^{i}\right) \leq \frac{\mu_{1}(\nu)^{\sigma}}{\left(\left(D \theta_{(i, e)}\right)\left(\tau_{1}\right)\right)^{\sigma}} \inf \left\{\sum_{j=1}^{k} \frac{\left(u_{\sigma}\right)_{\alpha(i, e)}\left(\theta_{(i, e)}\left(\zeta_{j}\right)\right)\left(\operatorname{diam}\left(A_{j}\right)\right)^{\sigma}\left(u_{\sigma}\right)_{i}\left(\zeta_{j}\right)}{\left(u_{\sigma}\right)_{\alpha(i, e)}\left(\theta_{(i, e)}\left(\zeta_{j}\right)\right)}:\right. \\
\left.\zeta_{j} \in \theta_{(i, e)}^{-1}\left(A_{j}\right)\right\} .
\end{array}
$$

Choose $\tau_{2} \in \theta_{(i, e)}^{-1}\left(N_{m \epsilon}\left(\theta_{(i, e)}\left(\Lambda_{\beta}^{i}\right)\right)\right)$ such that

$$
\frac{\left(u_{\sigma}\right)_{i}\left(\tau_{2}\right)}{\left(u_{\sigma}\right)_{\alpha(i, e)}\left(\theta_{(i, e)}\left(\tau_{2}\right)\right)} \geq \frac{\left(u_{\sigma}\right)_{i}\left(\zeta_{j}\right)}{\left(u_{\sigma}\right)_{\alpha(i, e)}\left(\theta_{(i, e)}\left(\zeta_{j}\right)\right)}
$$

for all $\zeta_{j} \in \theta_{(i, e)}^{-1}\left(A_{j}\right), 1 \leq j \leq k$. Using this together with (4.13), we get

$$
\tilde{\mathcal{H}}_{\epsilon^{*}, \eta}^{\sigma}\left(\Lambda_{\beta}^{i}\right) \leq \frac{\mu_{1}(\nu)^{\sigma}}{\left(\left(D \theta_{(i, e)}\right)\left(\tau_{1}\right)\right)^{\sigma}} \frac{\left(u_{\sigma}\right)_{i}\left(\tau_{2}\right)}{\left(u_{\sigma}\right)_{\alpha(i, e)}\left(\theta_{(i, e)}\left(\tau_{2}\right)\right)}\left(\tilde{\mathcal{H}}_{m \epsilon, \eta}^{\sigma}\left(\theta_{(i, e)}\left(\Lambda_{\beta}^{i}\right)\right)+\delta\right) .
$$

Since $\delta>0$ was arbitrary,

$$
\tilde{\mathcal{H}}_{\epsilon^{*}, \eta}^{\sigma}\left(\Lambda_{\beta}^{i}\right) \leq \frac{\mu_{1}(\nu)^{\sigma}}{\left(\left(D \theta_{(i, e)}\right)\left(\tau_{1}\right)\right)^{\sigma}} \frac{\left(u_{\sigma}\right)_{i}\left(\tau_{2}\right)}{\left(u_{\sigma}\right)_{\alpha(i, e)}\left(\theta_{(i, e)}\left(\tau_{2}\right)\right)} \tilde{\mathcal{H}}_{m \epsilon, \eta}^{\sigma}\left(\theta_{(i, e)}\left(\Lambda_{\beta}^{i}\right)\right) .
$$


The final step consists of replacing $\tau_{1}, \tau_{2}$ by a $\xi \in \Lambda_{\beta}^{i}$. From (4.17), it follows that

$$
\operatorname{diam}\left(\theta_{(i, e)}^{-1}\left(N_{m \epsilon}\left(\theta_{(i, e)}\left(\Lambda_{\beta}^{i}\right)\right)\right)\right) \leq \mu_{1}(\nu) \frac{1}{m} \nu \leq \kappa \nu
$$

where $\kappa$ is independent of $\nu$. In particular, we have that $d\left(\tau_{1}, \tau_{2}\right) \leq \kappa \nu$, so by continuity, there exists a function $\mu_{2}(\nu)$ such that $\mu_{2}(\nu) \rightarrow 1$ as $\nu \rightarrow 0$ and

$$
\mu_{2}(\nu)\left(\left(D \theta_{(i, e)}\right)(\xi)\right)^{\sigma} \frac{\left(u_{\sigma}\right)_{\alpha(i, e)}\left(\theta_{(i, e)}(\xi)\right)}{\left(u_{\sigma}\right)_{i}(\xi)} \leq\left(\left(D \theta_{(i, e)}\right)\left(\tau_{1}\right)\right)^{\sigma} \frac{\left(u_{\sigma}\right)_{\alpha(i, e)}\left(\theta_{(i, e)}\left(\tau_{2}\right)\right)}{\left(u_{\sigma}\right)_{i}\left(\tau_{2}\right)}
$$

$\xi \in \Lambda_{\alpha}^{i}$. Using this, 4.19) implies

$$
\tilde{\mathcal{H}}_{m \epsilon, \eta}^{\sigma}\left(\theta_{(i, e)}\left(\Lambda_{\beta}^{i}\right)\right) \geq \mu(\nu) \frac{\left(u_{\sigma}\right)_{\alpha(i, e)}\left(\theta_{(i, e)}(\xi)\right)}{\left(u_{\sigma}\right)_{i}(\xi)}\left(\left(D \theta_{(i, e)}\right)(\xi)\right)^{\sigma} \tilde{\mathcal{H}}_{\epsilon^{*}, \eta}^{\sigma}\left(\Lambda_{\beta}^{i}\right),
$$

where $\mu(\nu)=\mu_{2}(\nu)\left(\mu_{1}(\nu)\right)^{-\sigma}$. Now, we sum over $e \in E_{i}$, and use the fact that

$$
\sum_{e \in E_{i}}\left(\left(D \theta_{(i, e)}\right)(\xi)\right)^{\sigma}\left(u_{\sigma}\right)_{\alpha(i, e)}\left(\theta_{(i, e)}(\xi)\right)=\left(L_{\sigma} u_{\sigma}\right)_{i}(\xi)=r\left(L_{\sigma}\right)\left(u_{\sigma}\right)_{i}(\xi)
$$

to obtain (4.12).

Now we are ready to prove the remaining inequality.

Theorem 4.16. Assume that the hypotheses $\mathrm{H} 4.1, \mathrm{H} 4.2, \mathrm{H} 4.3$ and $\mathrm{H} 4.4$ are satisfied and let $C_{j} \subset S_{j}, 1 \leq j \leq p$, be the unique invariant list such that

$$
C_{i}=\bigcup_{(j, e) \in \Gamma_{i}} \theta_{(j, e)}\left(C_{j}\right) \text { for } 1 \leq i \leq p
$$

Also assume that $\left.\theta_{(j, e)}\right|_{C_{j}}$ is one-to-one for all $(j, e) \in \Gamma$ and that $\theta_{(j, e)}\left(C_{j}\right) \cap$ $\theta_{\left(j^{\prime}, e^{\prime}\right)}\left(C_{j^{\prime}}\right)=\emptyset$ whenever $\alpha(j, e)=\alpha\left(j^{\prime}, e^{\prime}\right)$ and $(j, e) \neq\left(j^{\prime}, e^{\prime}\right)$. Let $\sigma_{0}$ be the unique nonnegative real number such that $r\left(L_{\sigma_{0}}\right)=1$ and let $\beta_{0}$ denote the common Hausdorff dimension of $C_{i}$ for $1 \leq i \leq p$. Then $\beta_{0} \geq \sigma_{0}$.

Proof. We make the same notational conventions as in Lemma 4.15, If $B$ is any compact subset of $C_{j}, 1 \leq j \leq p$, and $\epsilon>0$, it is convenient to note that there is a positive, decreasing function $\phi(\epsilon)$, independent of $j$ and $B$, such that $\tilde{\mathcal{H}}_{\epsilon, \eta}^{\sigma}\left(B ; C_{j}\right):=$ $\tilde{\mathcal{H}}_{\epsilon, \eta}^{\sigma}(B)$ is defined whenever $0<\eta<\phi(\epsilon)$. The proof is left to the reader.

By Lemma 4.14 either (a) each $C_{j}$ is a compact, perfect metric space or (b) each $C_{j}$ is a single point. In case (b), our assumptions imply that for each $i, 1 \leq i \leq p$, there is a unique $(j, e) \in \Gamma$ with $\alpha(j, e)=i$. The linear map $L_{\sigma}$ then takes a simple form and the reader can verify that $\sigma_{0}=\beta_{0}=0$ in case (b). Thus we shall assume that we are in case (a).

Suppose $\beta_{0}<\sigma_{0}$. Then there exists a $\sigma<\sigma_{0}$ such that $\mathcal{H}^{\sigma}\left(C_{i}\right)=0$ for $1 \leq i \leq p$. This implies that for every $\epsilon>0, \mathcal{H}_{\epsilon}^{\sigma}\left(C_{i}\right)=0$ and using (4.7), we have

$$
\tilde{\mathcal{H}}_{\epsilon}^{\sigma}\left(C_{i}\right)=0 \text { for } \epsilon>0 \text {. }
$$

Let $(J, E)=\left[\left(j_{1}, e_{1}\right), \ldots,\left(j_{n}, e_{n}\right)\right] \in \Gamma^{(n)}$ and $\theta_{(J, E)}=\theta_{\left(j_{1}, e_{1}\right)} \circ \cdots \circ \theta_{\left(j_{n}, e_{n}\right)}$. Then $\operatorname{diam}\left(\theta_{(J, E)}\left(C_{j_{n}}\right)\right) \leq c^{n} \operatorname{diam}\left(C_{j_{n}}\right)$. So, given $\nu>0$, we can choose $n=n(\nu)$ large enough such that

$$
\operatorname{diam}\left(\theta_{(J, E)}\left(C_{j_{n}}\right)\right)<\nu \text { for all }(J, E) \in \Gamma^{(n)} .
$$


For $1 \leq i \leq p$, we have

$$
C_{i}=\bigcup_{(J, E) \in \Gamma_{i}^{(n)}} \theta_{(J, E)}\left(C_{j_{n}}\right)
$$

with the union being pairwise disjoint because of the disjointness assumption and the assumption that $\left.\theta_{(j, e)}\right|_{C_{j}}$ is one-to-one for $(j, e) \in \Gamma$. By Lemma 4.15, writing $\epsilon^{*}=\mu_{1}(\nu) \epsilon$,

$$
\mu(\nu) r\left(L_{\sigma}\right) \tilde{\mathcal{H}}_{\epsilon^{*}, \eta}^{\sigma}\left(\theta_{(J, E)}\left(C_{j_{n}}\right)\right) \leq \sum_{e \in E_{i}} \tilde{\mathcal{H}}_{m \epsilon, \eta}^{\sigma}\left(\theta_{(i, e)}\left(\theta_{(J, E)}\left(C_{j_{n}}\right)\right)\right)
$$

where $\mu(\nu) \rightarrow 1$ as $\nu \rightarrow 0$. Since $\mathcal{H}^{\sigma}\left(C_{j}\right)=0$ for $1 \leq j \leq p$, using Lemma 4.12, we get $\eta_{0}>0$ such that for $0<\eta<\eta_{0}$,

$$
\tilde{\mathcal{H}}_{m \epsilon, \eta}^{\sigma}\left(\theta_{(i, e)}\left(\theta_{(J, E)}\left(C_{j_{n}}\right)\right)\right)=\tilde{\mathcal{H}}_{\epsilon, \eta}^{\sigma}\left(\theta_{(i, e)}\left(\theta_{(J, E)}\left(C_{j_{n}}\right)\right)\right)
$$

and

$$
\tilde{\mathcal{H}}_{\epsilon^{*}, \eta}^{\sigma}\left(\theta_{(J, E)}\left(C_{j_{n}}\right)\right)=\tilde{\mathcal{H}}_{\epsilon, \eta}^{\sigma}\left(\theta_{(J, E)}\left(C_{j_{n}}\right)\right) .
$$

Therefore, the previous inequality becomes

$$
\mu(\nu) r\left(L_{\sigma}\right) \tilde{\mathcal{H}}_{\epsilon, \eta}^{\sigma}\left(\theta_{(J, E)}\left(C_{j_{n}}\right)\right) \leq \sum_{e \in E_{i}} \tilde{\mathcal{H}}_{\epsilon, \eta}^{\sigma}\left(\theta_{(i, e)}\left(\theta_{(J, E)}\left(C_{j_{n}}\right)\right)\right) .
$$

Now from (4.21), since the union is disjoint, we can choose $\epsilon>0$ so small that

$$
N_{\epsilon}\left(\theta_{(J, E)}\left(C_{j_{n}}\right)\right) \cap N_{\epsilon}\left(\theta_{\left(J^{\prime}, E^{\prime}\right)}\left(C_{j_{n}}\right)\right)=\emptyset
$$

for all $(J, E),\left(J^{\prime}, E^{\prime}\right) \in \Gamma_{i}^{(n)},(J, E) \neq\left(J^{\prime}, E^{\prime}\right)$. This implies that

$$
\tilde{\mathcal{H}}_{\epsilon, \eta}^{\sigma}\left(C_{i}\right)=\sum_{(J, E) \in \Gamma_{i}^{(n)}} \tilde{\mathcal{H}}_{\epsilon, \eta}^{\sigma}\left(\theta_{(J, E)}\left(C_{j_{n}}\right)\right)
$$

Therefore, we can sum (4.22) over all $(J, E) \in \Gamma_{i}^{(n)}$ to obtain

$$
\mu(\nu) r\left(L_{\sigma}\right) \tilde{\mathcal{H}}_{\epsilon, \eta}^{\sigma}\left(C_{i}\right) \leq \sum_{(J, E) \in \Gamma_{i}^{(n)}} \sum_{e \in E_{i}} \tilde{\mathcal{H}}_{\epsilon, \eta}^{\sigma}\left(\theta_{(i, e)}\left(\theta_{(J, E)}\left(C_{j_{n}}\right)\right)\right) .
$$

Now we sum over $i=1,2, \ldots, p$ to get

$$
\mu(\nu) r\left(L_{\sigma}\right) \sum_{i=1}^{p} \tilde{\mathcal{H}}_{\epsilon, \eta}^{\sigma}\left(C_{i}\right) \leq \sum_{i=1}^{p} \sum_{(J, E) \in \Gamma_{i}^{(n)}} \sum_{e \in E_{i}} \tilde{\mathcal{H}}_{\epsilon, \eta}^{\sigma}\left(\theta_{(i, e)}\left(\theta_{(J, E)}\left(C_{j_{n}}\right)\right)\right) .
$$

Note that $\theta_{(i, e)}\left(\theta_{(J, E)}\left(C_{j_{n}}\right)\right) \subset C_{\alpha(i, e)}$, so collecting the terms with $\alpha(i, e)=j$, $1 \leq j \leq p$, we get

$$
\mu(\nu) r\left(L_{\sigma}\right) \sum_{i=1}^{p} \tilde{\mathcal{H}}_{\epsilon, \eta}^{\sigma}\left(C_{i}\right) \leq \sum_{j=1}^{p} \sum_{(i, e) \in \Gamma_{j}} \sum_{(J, E) \in \Gamma_{i}^{(n)}} \tilde{\mathcal{H}}_{\epsilon, \eta}^{\sigma}\left(\theta_{(i, e)}\left(\theta_{(J, E)}\left(C_{j_{n}}\right)\right)\right) .
$$

Since $C_{j}=\bigcup_{(i, e) \in \Gamma_{j}} \bigcup_{(J, E) \in \Gamma_{i}^{(n)}} \theta_{(i, e)}\left(\theta_{(J, E)}\left(C_{j_{n}}\right)\right)$ with disjoint union, we get

$$
\mu(\nu) r\left(L_{\sigma}\right) \sum_{i=1}^{p} \tilde{\mathcal{H}}_{\epsilon, \eta}^{\sigma}\left(C_{i}\right) \leq \sum_{j=1}^{p} \tilde{\mathcal{H}}_{\epsilon, \eta}^{\sigma}\left(C_{j}\right)
$$

Since $\sigma<\sigma_{0}$, Lemma 4.6 implies that $r\left(L_{\sigma}\right)>1$, so we can choose $\nu>0$ small enough so that $\mu(\nu) r\left(L_{\sigma}\right)>1$. But, for $1 \leq i \leq p$, we have $\tilde{\mathcal{H}}_{\epsilon, \eta}^{\sigma}\left(C_{i}\right)>0$ by the definition of $\tilde{\mathcal{H}}_{\epsilon, \eta}^{\sigma}$. Also, we know that $\tilde{\mathcal{H}}_{\epsilon, \eta}^{\sigma}\left(C_{i}\right)$ is defined and finite for $\eta$ small 
enough. So, 4.23 cannot be true. Therefore, our initial assumption must be wrong. Thus, $\beta_{0} \geq \sigma_{0}$.

Combining Theorem 4.10 and Theorem 4.16, we have proved the following theorem.

Theorem 4.17. Assume that the hypotheses $\mathrm{H} 4.1, \mathrm{H} 4.2, \mathrm{H} 4.3$ and $\mathrm{H} 4.4$ are satisfied and let $C_{j} \subset S_{j}, 1 \leq j \leq p$, be the unique invariant list such that

$$
C_{i}=\bigcup_{(j, e) \in \Gamma_{i}} \theta_{(j, e)}\left(C_{j}\right) \text { for } 1 \leq i \leq p .
$$

Also assume that $\left.\theta_{(j, e)}\right|_{C_{j}}$ is one-to-one for all $(j, e) \in \Gamma$ and that $\theta_{(j, e)}\left(C_{j}\right) \cap$ $\theta_{\left(j^{\prime}, e^{\prime}\right)}\left(C_{j^{\prime}}\right)=\emptyset$ whenever $\alpha(j, e)=\alpha\left(j^{\prime}, e^{\prime}\right)$ and $(j, e) \neq\left(j^{\prime}, e^{\prime}\right)$. Let $\sigma_{0}$ be the unique nonnegative real number such that $r\left(L_{\sigma_{0}}\right)=1$. Then the Hausdorff dimension of each $C_{i}$ for $1 \leq i \leq p$ is the same, and if $\beta_{0}$ denotes the common Hausdorff dimension of $C_{i}$, then $\beta_{0}=\sigma_{0}$.

Remark 4.18. With the aid of Theorem 4.17 and elementary facts about positive linear operators, it is straightforward to prove that the Hausdorff dimension varies continuously with the functions $\theta_{(j, e)},(j, e) \in \Gamma$, in Theorem 4.17. The details will be given elsewhere, but we sketch the basic idea here. For each integer $m \geq 1$ and $(j, e) \in \Gamma$, suppose that $\theta_{(j, e), m}: S_{j} \rightarrow S_{\alpha(j, e)}$. Assume that, for $m \geq 1$, $\left\{\theta_{(j, e), m}:(j, e) \in \Gamma\right\}$ satisfies the assumptions of Theorem 4.17 and let $\sigma_{0, m}$ denote the corresponding Hausdorff dimension. For $(j, e) \in \Gamma$ and $x \in S_{j}$, assume that $\lim _{m \rightarrow \infty} \theta_{(j, e), m}(x)=\theta_{(j, e)}(x)$ and $\lim _{m \rightarrow \infty} D \theta_{(j, e), m}(x)=D \theta_{(j, e)}(x)$, where these limits define $\theta_{(j, e)}(x)$ and we assume that the limits are uniform in $x \in S_{j}$. Assume that $\theta_{(j, e)}$ satisfy $\mathrm{H} 4.1-\mathrm{H} 4.4$ and that $\left.\theta_{(j, e)}\right|_{C_{j}}$ is one-to-one, where $\left\{C_{j}: 1 \leq j \leq\right.$ $p\}$ is as in Theorem 2.3. For $\sigma \geq 0$, we have, in the obvious notation, linear operators $L_{\sigma, m}$ corresponding to $\left\{\theta_{(j, e), m}:(j, e) \in \Gamma\right\}$ and $L_{\sigma}$ corresponding to $\left\{\theta_{(j, e)}:(j, e) \in \Gamma\right\}$. If $\sigma_{0}$ denotes the unique value of $\sigma$ for which $r\left(L_{\sigma}\right)=1$, then $\lim _{m \rightarrow \infty} \sigma_{0, m}=\sigma_{0}$. If we assume, in addition, that $\theta_{(j, e)}\left(C_{j}\right) \cap \theta_{\left(j^{\prime}, e^{\prime}\right)}\left(C_{j^{\prime}}\right)=\emptyset$ whenever $(j, e) \neq\left(j^{\prime}, e^{\prime}\right)$ and $\alpha(j, e)=\alpha\left(j^{\prime}, e^{\prime}\right)$, then Theorem 4.17 implies that $\sigma_{0}$ is the Hausdorff dimension of $C_{j}$ for $1 \leq j \leq p$.

\section{INFINITE ITERATED FUNCTION SYSTEMS}

Let $(S, d)$ be a compact metric space, $I$ a countable set and for $i \in I$, let $\theta_{i}$ : $S \rightarrow S$ be a Lipschitz map with $\operatorname{Lip}\left(\theta_{i}\right) \leq c<1$. Following [12, pages 108-109], let $I^{\infty}$ denote the collection of all infinite sequences $\omega=\left(i_{1}, i_{2}, \ldots, i_{n}, \ldots\right)$, where $i_{n} \in I$ for all $n \geq 1$. Given $\omega=\left(i_{1}, i_{2}, \ldots, i_{n}, \ldots\right) \in I^{\infty}$ and $x \in S$, define $x_{n}(\omega)=\theta_{i_{1}} \circ \theta_{i_{2}} \circ \cdots \circ \theta_{i_{n}}(x)$ for $n \geq 1$. Just as in [12], one can easily prove that $\left\langle x_{n}(\omega): n \geq 1\right\rangle$ is a Cauchy sequence; the limit of this Cauchy sequence is independent of $x$, so we denote it by $\pi(\omega)$. We define $J=\left\{\pi(\omega): \omega \in I^{\infty}\right\}$. It is easy to show that

$$
J=\bigcup_{i \in I} \theta_{i}(J) .
$$

If $I^{\infty}$ is given the Cartesian product topology, then $\pi: I^{\infty} \rightarrow S$ is continuous. If $I$ is finite, $I^{\infty}$ is compact, so $\pi\left(I^{\infty}\right)=J$ is compact and (5.1) implies that $J$ must agree with our previous definition of the compact invariant set. However, if $I$ is infinite, $J$ may not be compact. 
For the remainder of this section, let $I=\mathbb{N}$ and let $\theta_{i}: S \rightarrow S$ be a Lipschitz map with $\operatorname{Lip}\left(\theta_{i}\right) \leq c<1$ for all $i \geq 1$. Let $J$ be the limit set associated with this infinite iterated function system as defined above. We wish to find a formula for the Hausdorff dimension of $J$. As in the case of finite iterated function systems, we study a Perron-Frobenius operator $L: C(S) \rightarrow C(S)$ of the form

$$
(L f)(t)=\sum_{i=1}^{\infty} b_{i}(t) f\left(\theta_{i}(t)\right) .
$$

We shall make the following assumptions.

H5.1 For $1 \leq i<\infty$, the function $b_{i}: S \rightarrow \mathbb{R}$ is nonnegative and continuous. Furthermore, for each $t \in S, b(t)=\sum_{i=1}^{\infty} b_{i}(t)<\infty$ and $b: S \rightarrow \mathbb{R}$ is continuous. H5.2 For $1 \leq i<\infty, \theta_{i}: S \rightarrow S$ is a Lipschitz map with $\operatorname{Lip}\left(\theta_{i}\right) \leq c<1$.

If H5.1 is satisfied and the maps $\theta_{i}$ are all continuous, it is easy to verify that $L$ defines a bounded linear map on $C(S)$. We refer the reader to Section 5 of [19] for a detailed discussion of such operators.

Let $K(M, \lambda)=\left\{f \in C(S): 0 \leq f(s) \leq f(t) \exp \left(M d(s, t)^{\lambda}\right)\right.$ for all $\left.s, t \in S\right\}$.

Lemma 5.1. Let $(S, d)$ be a compact metric space and let $L: C(S) \rightarrow C(S)$ be defined by (5.2). Assume that $\mathrm{H} 5.1$ and $\mathrm{H} 5.2$ are satisfied and assume that there exist $M_{0}>0$ and $\lambda>0$ such that for each $i \geq 1, b_{i} \in K\left(M_{0}, \lambda\right)$ and no $b_{i}$ is identically zero. Then there exists $M>0$ such that $L(K(M, \lambda)) \subset K(M, \lambda)$ and $L$ has a strictly positive eigenvector $u \in K(M, \lambda)$ with eigenvalue $r(L)>0$.

Proof. Since $c<1$, we can choose $M>0$ so that $M_{0}+M c^{\lambda} \leq M$. We claim that $L(K) \subset K$, where $K:=K(M, \lambda)$. Let $f \in K, i \geq 1$ and $s, t \in S$. Then we have

$$
\begin{gathered}
f\left(\theta_{i}(s)\right) \leq f\left(\theta_{i}(t)\right) \exp \left(M d\left(\theta_{i}(s), \theta_{i}(t)\right)^{\lambda}\right) \leq f\left(\theta_{i}(t)\right) \exp \left(M c^{\lambda} d(s, t)^{\lambda}\right) \text { and } \\
b_{i}(s) \leq b_{i}(t) \exp \left(M_{0} d(s, t)^{\lambda}\right) .
\end{gathered}
$$

Thus

$$
\begin{aligned}
(L f)(s) & =\sum_{i=1}^{\infty} b_{i}(s) f\left(\theta_{i}(s)\right) \\
& \leq \sum_{i=1}^{\infty} b_{i}(t) f\left(\theta_{i}(t)\right) \exp \left(\left(M_{0}+M c^{\lambda}\right) d(s, t)^{\lambda}\right) \\
& \leq(L f)(t) \exp \left(M d(s, t)^{\lambda}\right) .
\end{aligned}
$$

This proves that $L(K) \subset K$.

By Lemma 3.2. $\{f \in K:\|f\| \leq 1\}$ is equicontinuous, and hence it is compact by Ascoli's theorem. It follows that $\left.L\right|_{K}$ is a compact map. The constant function $e=1$ is in $K$, so

$$
r_{K}(L) \geq \lim _{n \rightarrow \infty}\left\|L^{n}(e)\right\|^{\frac{1}{n}}=r(L) .
$$

The opposite inequality is obviously true, so $r_{K}(L)=r(L)$. By assumption, $b(t)=$ $\sum_{i=1}^{\infty} b_{i}(t)>0$ for all $t \in S$, and $b: S \rightarrow \mathbb{R}$ is continuous by H5.1. Therefore, there exists $\delta>0$ with $\sum_{i=1}^{\infty} b_{i}(t) \geq \delta$. So $L(e) \geq \delta e$ from which it follows that $r(L) \geq \delta>0$. Since $\left.L\right|_{K}$ is a compact map and $r_{K}(L)=r(L)>0$, a theorem of Bonsall [1] implies that there exists $u \in K,\|u\|=1$, with $L(u)=r u, r=r(L)$. From the definition of $K(M, \lambda)$, it is clear that $u \in K \backslash\{0\}$ implies $u(t)>0$ for all $t \in S$. 
Remark 5.2. Many of the results of this section can be generalized to the case that $S$ is a bounded, complete, perfect metric space, but for simplicity we restrict to the case that $S$ is compact.

Throughout this section we shall make the following assumption.

H5.3 Let $(S, d)$ be a compact, perfect metric space and assume that for $1 \leq$ $i<\infty, \theta_{i}: S \rightarrow S$ is an infinitesimal similitude on $S$ and is a Lipschitz map with $\operatorname{Lip}\left(\theta_{i}\right) \leq c<1$. Assume that there exist $M_{0}>0$ and $\lambda_{0}>0$ such that for all $i \geq 1, D \theta_{i} \in K\left(M_{0}, \lambda\right)$ and $D \theta_{i}(t)>0$ for all $t \in S$. Assume, also, that there exist $\sigma>0$ and $t \in S$ with $\sum_{i=1}^{\infty}\left(D \theta_{i}(t)\right)^{\sigma}<\infty$.

Lemma 5.3. Assume $\mathrm{H} 5.3$ is satisfied. If $\sum_{i=1}^{\infty}\left(D \theta_{i}\left(t_{*}\right)\right)^{s}<\infty$ for some $t_{*} \in S$ and $s>0$, then for any $\sigma \geq s, \sum_{i=1}^{\infty}\left(D \theta_{i}(t)\right)^{\sigma}<\infty$ for all $t \in S$.

Proof. Since $D \theta_{i}(t)<1$ for all $t \in S,\left(D \theta_{i}(t)\right)^{\sigma} \leq\left(D \theta_{i}(t)\right)^{s}$ for $\sigma \geq s$. So $\sum_{i=1}^{\infty}\left(D \theta_{i}\left(t_{*}\right)\right)^{s}<\infty$ implies that $\sum_{i=1}^{\infty}\left(D \theta_{i}\left(t_{*}\right)\right)^{\sigma}<\infty$ for $\sigma \geq s$. Thus we only have to prove that for any $t \in S, \sum_{i=1}^{\infty}\left(D \theta_{i}(t)\right)^{s}<\infty$. Let $t \in S$. Since $D \theta_{i} \in K\left(M_{0}, \lambda\right), D \theta_{i}(t) \leq D \theta_{i}\left(t_{*}\right) \exp \left(M_{0} d\left(t, t_{*}\right)^{\lambda}\right)$. Therefore,

$$
\left(D \theta_{i}(t)\right)^{s} \leq\left(D \theta_{i}\left(t_{*}\right)\right)^{s} \exp \left(s M_{0} d\left(t, t_{*}\right)^{\lambda}\right),
$$

from which the result follows.

Assume H5.3 is satisfied. Define for $\sigma>0$ with $\sum_{i=1}^{\infty}\left(D \theta_{i}(t)\right)^{\sigma}<\infty$,

$$
\left(L_{\sigma} f\right)(t)=\sum_{i=1}^{\infty}\left(D \theta_{i}(t)\right)^{\sigma} f\left(\theta_{i}(t)\right) .
$$

Let $\sigma>0$ satisfying the above condition be fixed. If H5.1 is satisfied with $b_{i}(t):=$ $\left(D \theta_{i}(t)\right)^{\sigma}$ for $i \geq 1$, we know that $L_{\sigma}$ defines a bounded linear map on $C(S)$. By H5.3, $D \theta_{i} \in K\left(M_{0}, \lambda\right)$ for some $M_{0}>0, \lambda>0$. This implies that $b_{i} \in K\left(\sigma M_{0}, \lambda\right)$ for all $i \geq 1$. Therefore, if we choose $0<M<\infty$ such that $\sigma M_{0}+M c^{\lambda} \leq M$, $L_{\sigma}$ maps $K(M, \lambda)$ into itself and has an eigenvector $u_{\sigma} \in K(M, \lambda)$ with eigenvalue $r\left(L_{\sigma}\right)$.

Let $\sigma_{0}=\inf \left\{\sigma>0: \sum_{i=1}^{\infty}\left(D \theta_{i}(t)\right)^{\sigma}<\infty\right.$ for all $\left.t \in S\right\}$. If H5.3 holds, note that if $\sum_{i=1}^{\infty}\left(D \theta_{i}\left(t_{*}\right)\right)^{\sigma}<\infty$ for some $t_{*} \in S$, then $\sum_{i=1}^{\infty}\left(D \theta_{i}(t)\right)^{s}<\infty$ for all $t \in S$ and all $s>\sigma$. Assuming that $\mathrm{H} 5.3$ holds, there are two possible cases:

Case I. $\sum_{i=1}^{\infty}\left(D \theta_{i}(t)\right)^{\sigma}<\infty$ for all $\sigma>\sigma_{0}$ and all $t \in S$ but $\sum_{i=1}^{\infty}\left(D \theta_{i}(t)\right)^{\sigma_{0}}=\infty$ for all $t \in S$.

Case II. $\sum_{i=1}^{\infty}\left(D \theta_{i}(t)\right)^{\sigma}<\infty$ for all $\sigma \geq \sigma_{0}$ and all $t \in S$ but $\sum_{i=1}^{\infty}\left(D \theta_{i}(t)\right)^{\sigma}=\infty$ for all $\sigma<\sigma_{0}$ and all $t \in S$.

Note that $L_{\sigma}$ is defined for $\sigma>\sigma_{0}$ in Case I and for $\sigma \geq \sigma_{0}$ in Case II.

Lemma 5.4. The map $\sigma \mapsto r\left(L_{\sigma}\right)$ is strictly decreasing and continuous for $\sigma>\sigma_{0}$ in Case I and for $\sigma \geq \sigma_{0}$ in Case II. Also, $r\left(L_{\sigma}\right) \rightarrow 0$ as $\sigma \rightarrow \infty$.

Proof. The proof of strictly decreasing and continuity is exactly the same as the proof of Lemma 4.6. and we omit the details. To see the last part, fix $s>0$ with $\sup _{t \in S} \sum_{i=1}^{\infty}\left(D \theta_{i}(t)\right)^{s} \leq K<\infty$. Let $\sigma>s$. Then since $D \theta_{i}(t) \leq c$, $\sum_{i=1}^{\infty}\left(D \theta_{i}(t)\right)^{\sigma} \leq c^{\sigma-s} \sum_{i=1}^{\infty}\left(D \theta_{i}(t)\right)^{s}$. Therefore, $\left\|L_{\sigma}\right\| \leq \sup _{t \in S} \sum_{i=1}^{\infty}\left(D \theta_{i}(t)\right)^{\sigma}$ $\leq c^{\sigma-s} K$, which implies that $\left\|L_{\sigma}\right\| \rightarrow 0$ as $\sigma \rightarrow \infty$ because $c<1$. Since $r\left(L_{\sigma}\right) \leq\left\|L_{\sigma}\right\|$, the result follows. 
We should note that in the case of infinite iterated function systems there need not be a value of $\sigma$ for which $r\left(L_{\sigma}\right)=1$ because we cannot guarantee a $\sigma$ for which $r\left(L_{\sigma}\right) \geq 1$. It is possible that $r\left(L_{\sigma}\right)<1$ for all the values of $\sigma$ for which $L_{\sigma}$ is defined. Let

$$
\sigma_{\infty}=\inf \left\{\sigma>0: r\left(L_{\sigma}\right)<1\right\} .
$$

We claim that, under natural further assumptions, the Hausdorff dimension of the invariant set $J$ is equal to $\sigma_{\infty}$.

By Lemma 4.3 and Remark 4.4 we know that given $\epsilon>0$, there exists a $\mu_{i}(\epsilon)$ such that for every $t, s \in S$ with $0<d(s, t)<\epsilon$,

$$
\mu_{i}(\epsilon)^{-1}\left(D \theta_{i}\right)(t) \leq \frac{d\left(\theta_{i}(s), \theta_{i}(t)\right)}{d(s, t)} \leq \mu_{i}(\epsilon)\left(D \theta_{i}\right)(t)
$$

and $\lim _{\epsilon \rightarrow 0+} \mu_{i}(\epsilon)=1$. In the case of finitely many $\theta_{i}$ 's a uniform $\mu(\epsilon)$ satisfying the above property could be chosen by taking the maximum over $i$. But for the infinite case we cannot guarantee a uniform $\mu(\epsilon)$ which would work for each $\theta_{i}$. So instead we shall make the assumption that a uniform $\mu(\epsilon)$ can be chosen. For a specific problem we would have to check that this condition is indeed satisfied. For some important examples such as complex continued fractions, which have been studied by other authors (see Section 6 of [12]), this condition can be easily verified.

H5.4 Given $\epsilon>0$, there exists a $\mu(\epsilon) \geq 1$ such that for each $i \geq 1$ and for every $t, s \in S$ with $0<d(s, t)<\epsilon$,

$$
\mu(\epsilon)^{-1}\left(D \theta_{i}\right)(t) \leq \frac{d\left(\theta_{i}(s), \theta_{i}(t)\right)}{d(s, t)} \leq \mu(\epsilon)\left(D \theta_{i}\right)(t)
$$

and $\lim _{\epsilon \rightarrow 0+} \mu(\epsilon)=1$.

Now we are ready to prove the upper bound for the Hausdorff dimension of $J$.

Theorem 5.5. Assume that $\mathrm{H} 5.3$ and $\mathrm{H} 5.4$ are satisfied. Let $\operatorname{dim}(J)$ denote the Hausdorff dimension of the invariant set $J$ and $\sigma_{\infty}$ be as defined in (5.3). Then $\operatorname{dim}(J) \leq \sigma_{\infty}$.

Proof. Once we assume the existence of a uniform $\mu(\epsilon)$ as defined above, the proof is exactly similar (in fact, simpler since we are in the setting of iterated function systems) to the proof of Theorem 4.10 and is left to the reader.

To prove the other half, $\operatorname{dim}(J) \geq \sigma_{\infty}$, we shall consider the infinite iterated system as the limit of finite iterated systems and use the result that we have for the finite case.

Define for $N \geq 1$ and $f \in C(S)$,

$$
\left(L_{\sigma, N} f\right)(t)=\sum_{i=1}^{N}\left(D \theta_{i}(t)\right)^{\sigma} f\left(\theta_{i}(t)\right) .
$$

For $N \geq 1$, let $J_{N}$ be the unique nonempty compact invariant set satisfying

$$
J_{N}=\bigcup_{i=1}^{N} \theta_{i}\left(J_{N}\right)
$$

and let $\sigma_{N}$ be the unique positive real number such that $r\left(L_{\sigma_{N}, N}\right)=1$. We shall assume the following hypothesis. 
H5.5 For each $N \geq 1, \theta_{i}\left(J_{N}\right) \cap \theta_{j}\left(J_{N}\right)=\emptyset$ for $1 \leq i<j \leq N$, and $\left.\theta_{i}\right|_{J_{N}}$ is one-to-one for $1 \leq i \leq N$.

By Theorem 4.17 we know that, assuming $\mathrm{H} 5.3$ and $\mathrm{H} 5.5, \operatorname{dim}\left(J_{N}\right)=\sigma_{N}$.

Lemma 5.6. For each $N \geq 1, J_{N} \subset J_{N+1}$ and $J_{N} \subset J$. Hence, $\operatorname{dim}(J) \geq$ $\operatorname{dim}\left(J_{N}\right)=\sigma_{N}$.

Proof. We know that $J_{N}=\left\{\pi(\omega): \omega \in\{1,2, \ldots, N\}^{\infty}\right\}$ for any $N \geq 1$, where $\pi$ is the map defined in the beginning of this section. It follows that $J_{N} \subset J_{N+1}$ for all $N \geq 1$. Also since $J=\left\{\pi(\omega): \omega \in \mathbb{N}^{\infty}\right\}$, we have $J_{N} \subset J$ for all $N \geq 1$. This completes the proof of the lemma.

It is easy to see that $\left\|L_{\sigma}-L_{\sigma, N}\right\| \rightarrow 0$ as $N \rightarrow \infty$ for $\sigma>\sigma_{0}$ in Case I and for $\sigma \geq \sigma_{0}$ in Case II.

Remark 5.7. Let $X$ be a real or complex Banach space and $L: X \rightarrow X, L_{k}$ : $X \rightarrow X, k \geq 1$ be bounded linear operators. Assume that $\lim _{k \rightarrow \infty}\left\|L_{k}-L\right\|=0$. Then we have that $\limsup _{k \rightarrow \infty} r\left(L_{k}\right) \leq r(L)$. But, in general, it is not true that $\lim _{k \rightarrow \infty} r\left(L_{k}\right) \rightarrow r(L)$. In fact, Kakutani has given an example of a sequence of bounded linear operators $L_{k}$ on a Hilbert space which converges in the operator norm to an operator $L$ and satisfies $r\left(L_{k}\right)=0$ for all $k \geq 1$ and $r(L)>0$. The example can be found on pages 282-283 of 20. If, in addition, we know that $\rho(L)<r(L)$, where $\rho(L)$ is the essential spectral radius of $L$, then it is true that $r\left(L_{k}\right) \rightarrow r(L)$. To see this, note that by using the natural extension of $L$ to the complexification of $X$, we can assume that $X$ is a complex Banach space. If $\sigma(L)$ denotes the spectrum of $L$, recall that $\sigma(L) \cap\{z \in \mathbb{C}:|z|>\rho(L)\}$ consists of isolated points, each of which is an eigenvalue of $L$ of finite algebraic multiplicity. Then exactly the argument on pages 227-228 of [17] proves that $r\left(L_{k}\right) \rightarrow r(L)$.

The following lemma is known. The proof is included for the reader's convenience.

Lemma 5.8. Let $(S, d)$ be a compact metric space and suppose that $L: X=$ $C(S) \rightarrow X$ is a positive bounded linear map; i.e., $f(t) \geq 0$ for all $t \in S$ implies that $(L f)(t) \geq 0$ for all $t \in S$. Let e denote the function identically equal to 1 . If $r(L)$ denotes the spectral radius of $L$, we have $r(L)=\lim _{n \rightarrow \infty}\left\|L^{n} e\right\|^{\frac{1}{n}}$. Furthermore, if $u \in X$ is such that $u(t)>0$ for all $t \in S$, then $r(L)=\lim _{n \rightarrow \infty}\left\|L^{n} u\right\|^{\frac{1}{n}}$. Finally, if $L u=r u$ with $u(t)>0$ for all $t \in S$, then $r(L)=r$.

Proof. We shall write $f \leq g$ to mean $f(t) \leq g(t)$ for all $t \in S$. Since $L$ is linear and maps nonnegative functions to nonnegative functions, it follows that $L f \leq L g$ whenever $f \leq g$. If $f \in X$ with $\|f\| \leq 1$, we have $-e \leq f \leq e$. So, $-L^{n} e \leq L^{n} f \leq$ $L^{n} e$, which implies that $\left|\left(L^{n} f\right)(t)\right| \leq\left|L^{n} e(t)\right|$ for all $t \in S$. Thus $\left\|L^{n} f\right\| \leq\left\|L^{n} e\right\|$ whenever $\|f\| \leq 1$, which gives $\left\|L^{n}\right\|=\left\|L^{n} e\right\|$. Taking the $n$th root and taking the limit as $n$ goes to $\infty$, we get $r(L)=\lim _{n \rightarrow \infty}\left\|L^{n} e\right\|^{\frac{1}{n}}$.

Now let $u \in X$ such that $u(t)>0$ for all $t \in S$. Since $S$ is compact, there exist $0<m<M<\infty$ such that $m e \leq u \leq M e$. This implies $m L^{n} e \leq L^{n} u \leq M L^{n} e$, so $m\left\|L^{n} e\right\| \leq\left\|L^{n} u\right\| \leq M\left\|L^{n} e\right\|$. Taking the $n$th root and taking the limit, we get $\lim _{n \rightarrow \infty}\left\|L^{n} u\right\|^{\frac{1}{n}}=\lim _{n \rightarrow \infty}\left\|L^{n} e\right\|^{\frac{1}{n}}=r(L)$. To see the last part, note that $L u=r u$ implies $L^{n} u=r^{n} u$. So, $\left\|L^{n} u\right\|^{\frac{1}{n}}=r\|u\|^{\frac{1}{n}}$. Since $\|u\|>0,\|u\|^{\frac{1}{n}} \rightarrow 1$. Thus we get $r(L)=r$. 
Corollary 5.9. Let $(S, d)$ be a compact metric space and suppose that $L: X=$ $C(S) \rightarrow X$ and $L_{k}: X \rightarrow X$ are positive bounded linear maps. Assume that $\left\|L_{k}-L\right\| \rightarrow 0$ as $k \rightarrow \infty$. Suppose $L u=$ ru with $u(t)>0$ for all $t \in S$. Then $r\left(L_{k}\right) \rightarrow r(L)=r$.

Proof. First we know that $r=r(L)$ by Lemma 5.8. Now we have that $\left\|L_{k} u-L u\right\| \rightarrow$ 0 as $k \rightarrow \infty$. Because $u$ is strictly positive, given $\delta>0$, there exists $k_{0}$ such that $L_{k} u \geq(1-\delta) r u$ for all $k \geq k_{0}$. This implies that for any $n \geq 1,\left\|L_{k}^{n} u\right\| \geq$ $(1-\delta)^{n} r^{n}\|u\|$ for $k \geq k_{0}$. By Lemma 5.8 $r\left(L_{k}\right)=\lim _{n \rightarrow \infty}\left\|L_{k}^{n} u\right\|^{\frac{1}{n}} \geq(1-\delta) r$ for $k \geq k_{0}$. Since $\delta>0$ was arbitrary, $\liminf _{k \rightarrow \infty} r\left(L_{k}\right) \geq r$. Thus we are done because we always have $\lim \sup _{k \rightarrow \infty} r\left(L_{k}\right) \leq r(L)$.

Lemma 5.10. Assume that $\mathrm{H} 5.3$ and $\mathrm{H} 5.4$ are satisfied. Then we have that $r\left(L_{\sigma, N}\right) \uparrow r\left(L_{\sigma}\right)$ as $N \rightarrow \infty$ for $\sigma>\sigma_{0}$ in Case I and for $\sigma \geq \sigma_{0}$ in Case II.

Proof. Let $\sigma>\sigma_{0}$ if we are in Case I or $\sigma \geq \sigma_{0}$ if we are in Case II. If $b_{i}(t)=$ $\left(D \theta_{i}(t)\right)^{\sigma}$, then H5.3 implies that $b_{i} \in K\left(\sigma M_{0}, \lambda\right)$, and using this fact and H5.3, the reader can verify that $b(t):=\sum_{i=1}^{\infty} b_{i}(t)>0$ for all $t \in S$ and $b$ is continuous. It follows that $L_{\sigma}$ defines a positive bounded linear operator on $C(S)$. Clearly $L_{\sigma, N} e \leq L_{\sigma, N+1} e$, so $\left\|L_{\sigma, N} e\right\| \leq\left\|L_{\sigma, N+1} e\right\|$. By Lemma 5.8 we get that $r\left(L_{\sigma, N}\right) \leq$ $r\left(L_{\sigma, N+1}\right)$. It is easy to see, using H5.3, that $\left\|L_{\sigma}-L_{\sigma, N}\right\| \rightarrow 0$ as $N \rightarrow \infty$. Since we know that $L_{\sigma}$ has a strictly positive eigenvector with eigenvalue $r\left(L_{\sigma}\right)$, by Corollary [5.9, we must have $r\left(L_{\sigma, N}\right) \rightarrow r\left(L_{\sigma}\right)$.

Now we can prove the lower bound for the Hausdorff dimension of $J$.

Theorem 5.11. Assume that $\mathrm{H} 5.3, \mathrm{H} 5.4$ and $\mathrm{H} 5.5$ are satisfied. Let $\operatorname{dim}(J)$ denote the Hausdorff dimension of $J$ and $\sigma_{\infty}$ be as defined in (5.3). Then $\operatorname{dim}(J)=\sigma_{\infty}$.

Proof. By Theorem [5.5] it suffices to prove that $\operatorname{dim}(J) \geq \sigma_{\infty}$. If $\sigma_{0}<\sigma_{\infty}$, then for $\sigma_{0}<\sigma<\sigma_{\infty}, L_{\sigma}$ is defined and $r\left(L_{\sigma}\right)>1$ by the definition of $\sigma_{\infty}$ and the fact that $\sigma \mapsto r\left(L_{\sigma}\right)$ is strictly decreasing. So, by Lemma [5.10, there exists $N_{\sigma}$ such that $r\left(L_{\sigma, N}\right)>1$ for all $N \geq N_{\sigma}$. Since $r\left(L_{\sigma_{N}, N}\right)=1, \sigma_{N}>\sigma$ for all $N \geq N_{\sigma}$. Therefore, $\operatorname{dim}(J) \geq \operatorname{dim}\left(J_{N_{\sigma}}\right)=\sigma_{N_{\sigma}}>\sigma$. Since this is true for any $\sigma$ with $\sigma_{0}<\sigma<\sigma_{\infty}$, it follows that $\operatorname{dim}(J) \geq \sigma_{\infty}$. If $\sigma_{0}=\sigma_{\infty}$, then for $\sigma<\sigma_{\infty}$, $\sum_{i=1}^{\infty}\left(D \theta_{i}(t)\right)^{\sigma}=\infty$, so for large $N$ (depending on $\sigma$ ), $\sum_{i=1}^{N}\left(D \theta_{i}(t)\right)^{\sigma}>1$ for all $t \in S$. This implies that $r\left(L_{\sigma, N}\right)>1$, so $\sigma_{N}>\sigma$. Thus $\operatorname{dim}(J)>\sigma$ for all $\sigma<\sigma_{\infty}$, and hence $\operatorname{dim}(J) \geq \sigma_{\infty}$. This completes the proof of the theorem.

To illustrate Theorem 5.11, we discuss a special infinite iterated function system that is generated by complex continued fractions. This has been studied in section 6 of [12]. We show how our theory is applicable to this particular example.

Example 5.12 (Complex continued fractions). Let $I$ be an infinite subset of $\{m+$ $n i: m \in \mathbb{N}, n \in \mathbb{Z}\}$, where $\mathbb{Z}$ is the set of integers and $\mathbb{N}$ is the set of positive integers. Let $X \subset \mathbb{C}$ be the closed disc centered at the point $\frac{1}{2}$ with radius $\frac{1}{2}$. For $b \in I$ define $\theta_{b}: X \rightarrow X$ by

$$
\theta_{b}(z)=\frac{1}{b+z}
$$

The mappings $\theta_{b}, b \in I$, may not all be strict contractions in the Euclidean metric; $\theta_{1}$ is not a strict contraction because $\left|\theta_{1}^{\prime}(0)\right|=1$. Therefore we consider the system $\left\{\theta_{b} \circ \theta_{c}: b, c \in I\right\}$. It is easy to verify that $\theta_{b} \circ \theta_{c}$ is a strict contraction for each $b, c \in I$ with a uniform Lipschitz constant $\kappa<1$. Let $J$ be the invariant set for 
this system as defined in this section. First note that $\theta_{b}(z)=\theta_{c}(w)$ implies that $|z-w|=|b-c|$. So, if $|b-c|>1$, then $\theta_{b}(X) \cap \theta_{c}(X)=\emptyset$. Furthermore, if $|b-c|=1$, then $\theta_{b}(z)=\theta_{c}(w)$ implies that $z$ and $w$ belong to the boundary of $X$ and $|z-w|=1$.

Lemma 5.13. $\theta_{b} \circ \theta_{c}(X)$ is contatined in the interior of $X$.

Proof. First we claim that $\theta_{b}(z) \in \partial X$ implies $z=0$. Let $b=m+n i, m \in \mathbb{N}$, $n \in \mathbb{Z}$ and $z=x+y i \in X$. Then $\theta_{b}(z) \in \partial X$ implies $\left|\frac{1}{b+z}-\frac{1}{2}\right|=\frac{1}{2}$, which implies $|2-b-z|=|b+z|$. Therefore, $(2-m-x)^{2}+(n+y)^{2}=(m+x)^{2}+(n+y)^{2}$, which implies $m+x=1$, i.e., $x=1-m$. Since $m \geq 1$ and $x \geq 0$ for $z \in X$, it follows that $m=1$ and $x=0$. But $x=0$ implies that $z=0$.

Now suppose that $\theta_{b} \circ \theta_{c}(z) \in \partial X$ for some $z \in X$. Then, by the above claim, $\theta_{c}(z)=0$, which is impossible by the definition of $\theta_{c}(z)$.

Let us verify H5.5. By the previous lemma, we know that for any $b, c \in I$, $\theta_{b} \circ \theta_{c}(X)$ is a compact set contained in the interior of $X$. So if we take finitely many $b_{i}, c_{i}$, the union of the images would still be a compact subset of the interior of $X$. So, for any $N, J_{N}$ is a compact subset of the interior of $X$, which means that $\operatorname{diam}\left(J_{N}\right)<1$. We claim that if $\hat{X} \subset \operatorname{int}(X)$ with $\operatorname{diam}(\hat{X})<1$, then $\theta_{b_{1}} \circ \theta_{c_{1}}(\hat{X}) \cap$ $\theta_{b_{2}} \circ \theta_{c_{2}}(\hat{X})=\emptyset$ for any $\left(b_{1}, c_{1}\right) \neq\left(b_{2}, c_{2}\right)$. Suppose instead that $\theta_{b_{1}}\left(\theta_{c_{1}}(z)\right)=$ $\theta_{b_{2}}\left(\theta_{c_{2}}(w)\right)$ with $z, w \in \hat{X}$. This implies that $b_{1}+\theta_{c_{1}}(z)=b_{2}+\theta_{c_{2}}(w)$. If $b_{1}=b_{2}$, this would imply that $\theta_{c_{1}}(z)=\theta_{c_{2}}(w)$, i.e., $c_{1}+z=c_{2}+w$, which is impossible because $|z-w|<1$ and $\left|c_{1}-c_{2}\right| \geq 1$. If $b_{1} \neq b_{2}$, we must have $\left|\theta_{c_{1}}(z)-\theta_{c_{2}}(w)\right|=1$, which is possible only if both $\theta_{c_{1}}(z)$ and $\theta_{c_{2}}(w)$ belong to the boundary of $X$, which is possible only if $z=w=0$. This is a contradiction to the fact that $\hat{X}$ is in the interior of $X$. Thus the disjointness condition in H5.5 is satisfied. Also for any $b \in I$, the map $\theta_{b}$ is clearly one-to-one.

For $b \in I$, we have $D \theta_{b}(z)=\left|\theta^{\prime}(z)\right|=\frac{1}{|z+b|^{2}}$. So, $D \theta_{b}(z)>0$ for all $z \in X$. We claim that there exists $0<M_{0}<\infty$ such that $D \theta_{b} \in K\left(M_{0}, \lambda\right)$ with $\lambda=1$. Let $z, w \in X$. We have

$$
\begin{gathered}
\left(D \theta_{b}\right)(z) \leq\left(D \theta_{b}\right)(w) \exp \left(M_{0}|z-w|\right) \\
\Leftrightarrow \frac{1}{|z+b|^{2}} \leq \frac{1}{|w+b|^{2}} \exp \left(M_{0}|z-w|\right) \\
\Leftrightarrow M_{0} \geq 2 \frac{\ln \left|\frac{w+b}{z+b}\right|}{|w-z|} .
\end{gathered}
$$

But $\ln \left|\frac{w+b}{z+b}\right|=\ln \left|1+\frac{w-z}{z+b}\right| \leq \ln \left(1+\frac{|w-z|}{|z+b|}\right) \leq \frac{|w-z|}{|z+b|}$. Therefore, $2 \frac{\ln \left|\frac{w+b}{z+b}\right|}{|w-z|} \leq \frac{2}{|z+b|} \leq$ 2. So, we can choose $M_{0}$ independent of $b \in I$.

To complete the verification of H5.3 note that

$$
\sum_{b \in I}\left(D \theta_{b}\right)^{\sigma}(0)=\sum_{b \in I} \frac{1}{|b|^{2 \sigma}} \leq \sum_{n \in \mathbb{Z}} \sum_{m \in \mathbb{N}} \frac{1}{\left(m^{2}+n^{2}\right)^{2 \sigma}},
$$

which converges for $\sigma>1$.

To verify H5.4 it is enough to show that given $\epsilon>0$ there exist $\mu_{1}(\epsilon)$ and $\mu_{2}(\epsilon)$ such that $\lim _{\epsilon \rightarrow 0+} \mu_{1}(\epsilon)=\lim _{\epsilon \rightarrow 0+} \mu_{2}(\epsilon)=1$ and if $0<|z-w|<\epsilon$, then $\mu_{1}(\epsilon) \leq \frac{1}{\left(D \theta_{b}\right)(z)} \frac{\left|\theta_{b}(z)-\theta_{b}(w)\right|}{|z-w|} \leq \mu_{2}(\epsilon)$ for all $b \in I$. But using $\theta_{b}(z)=\frac{1}{z+b}$, we get $\frac{1}{\left(D \theta_{b}\right)(z)} \frac{\left|\theta_{b}(z)-\theta_{b}(w)\right|}{|z-w|}=\left|\frac{z+b}{w+b}\right|=\left|1+\frac{z-w}{w+b}\right|$, which is bounded between $1-\frac{|z-w|}{|w+b|}$ and 
$1+\frac{|z-w|}{|w+b|}$. Since for any $w \in X,|w+b|^{-1}=\left|\theta_{b}(w)\right| \leq 1$, taking $\mu_{1}(\epsilon)=1-\epsilon$ and $\mu_{2}(\epsilon)=1+\epsilon$ does the job.

Thus we see that all the hypotheses of the theorem are satisfied for this particular example, and hence the Hausdorff dimension of the invariant set is given by the value of $\sigma_{\infty}$. Simpler versions of these arguments show that the results of $\S 4$ are applicable if $I$ is a finite set.

In the next section we shall see that sometimes it is important to look at another metric rather than the Euclidean metric.

\section{Choice of Appropriate Metric}

We need to recall the definition of the Carathéodory-Reiffen-Finsler (CRF) metric on bounded domains in Banach spaces. Let $G$ be a bounded domain in a complex Banach space $X$ and let $U$ denote the open unit disc in $\mathbb{C}$. Let $\operatorname{Hol}(G, U)$ be the family of all holomorphic functions $f: G \rightarrow U$. Define $\alpha: G \times X \rightarrow \mathbb{R}$ by

$$
\alpha(x, v)=\sup \{|D g(x) v|: g \in \operatorname{Hol}(G, U)\},
$$

where $D g(x)$ denotes the Fréchet derivative of $g$ at $x$. Given any two points $x$ and $y$ in $G$, consider the family of curves $\gamma:[0,1] \rightarrow G$ that have piecewise continuous derivatives and $\gamma(0)=x, \gamma(1)=y$. Call such a curve admissible and define its length by

$$
L(\gamma)=\int_{0}^{1} \alpha\left(\gamma(t), \gamma^{\prime}(t)\right) d t
$$

We now define the distance between $x$ and $y$ by

$$
\rho(x, y)=\inf \{L(\gamma): \gamma \text { is admissible with } \gamma(0)=x \text { and } \gamma(1)=y\} .
$$

$\rho$ is called the CRF metric on $G$. For a detailed discussion of the CRF metric we refer the reader to $[6]$.

Let $G$ be a bounded open set in $\mathbb{C}$ and let $\theta: G \rightarrow G$ be a holomorphic map such that $\overline{\theta(G)}$ is a compact subset of $G$. If $\rho$ denotes the CRF metric on $G$, then it is known (see Theorem 13.1 in [6]) that $\theta$ is a strict contraction on $G$ with respect to the CRF metric $\rho$. Also, on a compact subset $C$ of $G, \rho$ is a complete metric and is equivalent to the standard Euclidean metric; i.e., there exist positive constants $m$ and $M$ such that

$$
m|z-w| \leq \rho(z, w) \leq M|z-w| \text { for all } z, w \text { in } C .
$$

Let $G$ be a bounded open set in $\mathbb{C}$ and assume for $1 \leq i \leq N$ that $\theta_{i}: G \rightarrow G$ is a holomorphic map such that $C_{i}=\overline{\theta_{i}(G)}$ is a compact subset of $G$ and $\theta_{i}^{\prime}(z) \neq 0$ for all $z \in G$. Define $C:=\bigcup_{i=1}^{N} C_{i}$. Then, by Theorem 2.3 and the above remarks, there exists a unique nonempty compact set $K$ with $K=\bigcup_{i=1}^{N} \theta_{i}(K)$. For $k \geq 1$, define $\mathcal{I}_{k}=\left\{I=\left(i_{1}, i_{2}, \ldots, i_{k}\right): 1 \leq i_{j} \leq N\right.$ for $\left.1 \leq j \leq k\right\}$. For $I=\left(i_{1}, i_{2}, \ldots, i_{k}\right) \in \mathcal{I}_{k}$, define $\theta_{I}=\theta_{i_{k}} \circ \cdots \circ \theta_{i_{2}} \circ \theta_{i_{1}}$. It is easy to see that $K=\bigcup_{I \in \mathcal{I}_{k}} \theta_{I}(K)$. We claim that, for large $k, \theta_{I}$ is a strict contraction map with respect to the Euclidean metric. Suppose that $z, w \in C, z \neq w$. Then

$$
\frac{\left|\theta_{I}(z)-\theta_{I}(w)\right|}{|z-w|} \leq \frac{\frac{1}{m} \rho\left(\theta_{I}(z), \theta_{I}(w)\right)}{\frac{1}{M} \rho(z, w)} \leq \frac{M}{m} c^{k},
$$

where $c<1$ is the maximum of the contraction ratios of the maps $\theta_{i}, 1 \leq i \leq N$, with respect to the metric $\rho$. If we choose $k$ large enough so that $\frac{M}{m} c^{k}<1$, then 
it follows that $\theta_{I}$ is a contraction map for all $I \in \mathcal{I}_{k}$ with respect to the Euclidean metric. Thus, if $\theta_{I}(K) \cap \theta_{J}(K)=\emptyset$ for $I, J \in \mathcal{I}_{k}, I \neq J$ (which is certainly true if $\left\{\theta_{i}(K)\right\}_{i=1}^{N}$ are pairwise disjoint and $\left.\theta_{i}\right|_{K}$ is one-to-one for $1 \leq i \leq N$ ), the Hausdorff dimension of the invariant set $K$ is given by Theorem 1.2 by considering the iterated function system given by the maps $\left\{\theta_{I}\right\}_{I \in \mathcal{I}_{k}}$ and the standard Euclidean metric. Note that in this case $\left(D \theta_{I}\right)(z)$ is nothing but $\left|\theta_{I}^{\prime}(z)\right|$. If we write, for $\sigma \geq 0$, $\left(L_{\sigma} f\right)(z)=\sum_{i=1}^{N}\left|\theta_{i}^{\prime}(z)\right|^{\sigma} f\left(\theta_{i}(z)\right)$ and $\left(\tilde{L}_{\sigma} f\right)(z)=\sum_{I \in \mathcal{I}_{k}}\left|\theta_{I}^{\prime}(z)\right|^{\sigma} f\left(\theta_{I}(z)\right)$, where $k$ is as chosen above, then it is easy to see that $\tilde{L}_{\sigma}=L_{\sigma}^{k}$. It follows from Lemma 6.1 below that $r\left(\tilde{L}_{\sigma_{0}}\right)=1$ if and only if $r\left(L_{\sigma_{0}}\right)=1$. Thus, to find the Hausdorff dimension of the invariant set $K$, it is enough to find $\sigma_{0}$ such that $r\left(L_{\sigma_{0}}\right)=1$.

The following lemma is well known, and the proof is given only for completeness.

Lemma 6.1. Let $X$ be a Banach space, $L: X \rightarrow X$ be a bounded linear map and $k \geq 1$ be a positive integer. Then $r\left(L^{k}\right)=(r(L))^{k}$, where $r(L)$ denotes the spectral radius of $L$.

Proof. We have that

$$
\begin{aligned}
r\left(L^{k}\right) & =\lim _{n \rightarrow \infty}\left\|\left(L^{k}\right)^{n}\right\|^{1 / n} \\
& =\lim _{n \rightarrow \infty}\left(\left\|L^{k n}\right\|^{1 / k n}\right)^{k} \\
& =\left(\lim _{n \rightarrow \infty}\left\|L^{k n}\right\|^{1 / k n}\right)^{k} \\
& =(r(L))^{k} .
\end{aligned}
$$

\section{REFERENCES}

1. F. F. Bonsall, Linear operators in complete positive cones, Proc. London Math. Soc. 8 (1958), 53-75. MR0092938 (19:1183c)

2. R. T. Bumby, Hausdorff dimensions of Cantor sets, J. Reine Angew. Math. 331 (1982), 192-206. MR647383 (83g:10038)

3. _ Hausdorff dimension of sets arising in number theory, Number theory (New York, 1983-84), Lecture Notes in Math., vol. 1135, Springer, Berlin, 1985, pp. 1-8. MR803348 (87a:11074)

4. G. Edgar, Measure, Topology and Fractal Geometry, second ed., Undergraduate Texts in Mathematics, Springer, New York, 2008. MR2356043 (2009e:28001)

5. K. J. Falconer, The Geometry of Fractal Sets, Cambridge Univ. Press, Cambridge, 1985.

6. K. Goebel and S. Reich, Uniform Convexity, Hyperbolic Geometry, and Nonexpansive Mappings, Monographs and Textbooks in Pure and Applied Mathematics, vol. 83, Marcel Dekker Inc., New York, 1984. MR744194 (86d:58012)

7. J. E. Hutchinson, Fractals and self-similarity, Indiana Univ. Math. J. 30 (1981), no. 5, 713747. MR625600 (82h:49026)

8. M. G. Krein and M. A. Rutman, Linear operators leaving invariant a cone in a Banach space (in Russian), Uspehi Matem. Nauk 3 (1948), no. 1(23), 3-95. MR0027128 (10:256c)

9. J. Mallet-Paret and R. D. Nussbaum, Eigenvalues for a class of homogeneous cone maps arising from max-plus operators, Discrete Contin. Dyn. Syst. 8 (2002), no. 3, 519-562. MR.1897866 (2003c:47088)

10. , Generalizing the Krein-Rutman theorem, measures of noncompactness and the fixed point index, J. Fixed Point Theory and Applications 7 (2010), 103-143.

11. P. Mattila, Geometry of Sets and Measures in Euclidean Spaces, Cambridge Univ. Press, Cambridge, 1995. MR.1333890 (96h:28006)

12. R. D. Mauldin and M. Urbański, Dimensions and measures in infinite iterated function systems, Proc. London Math. Soc. (3) (1996), no. 1, 105-154. MR1387085 (97c:28020) 
13. R. D. Mauldin and S. C. Williams, Hausdorff dimension in graph directed constructions, Trans. Amer. Math. Soc. 309 (1988), no. 2, 811-829. MR961615 (89i:28003)

14. S. Mazur and S. Ulam, Sur les transformations isométriques d'espaces vectoriels normés, C. R. Acad. Sci. Paris 194 (1932), 946-948.

15. P. A. P. Moran, Additive functions of intervals and Hausdorff measure, Proc. Cambridge Philos. Soc. 42 (1946), 15-23. MR0014397 (7:278f)

16. J. R. Munkres, Topology, second ed., Prentice Hall, 2000.

17. R. D. Nussbaum, Periodic solutions of some nonlinear integral equations, Dynamical systems (Proc. Internat. Sympos., Univ. Florida, Gainesville, Fla., 1976), Academic Press, New York, 1977, pp. 221-249. MR0463844 (57:3783)

18. _ Eigenvectors of nonlinear positive operators and the linear Krein-Rutman theorem, Fixed point theory, Lecture Notes in Math., vol. 886, Springer, Berlin, 1981, pp. 309-330. MR643014 (83b:47068)

19. , Periodic points of positive linear operators and Perron-Frobenius operators, Integral Equations Operator Theory 39 (2001), no. 1, 41-97. MR.1806843 (2001m:47083)

20. C. E. Rickart, General Theory of Banach Algebras, Robert E. Krieger Publishing Co., New York, 1974.

21. H. H. Schaefer, Banach Lattices and Positive Operators, Springer-Verlag, New York, 1974. MR:0423039(54:11023)

22. A. Schief, Self-similar sets in complete metric spaces, Proc. Amer. Math. Soc. 124 (1996), no. 2, 481-490. MR.1301047(96h:28015)

23. J. Väisälä, A proof of the Mazur-Ulam theorem, Amer. Math. Monthly 110 (2003), no. 7, 633-635. MR2001155 (2004d:46021)

Department of Mathematics, Rutgers University, Piscataway, New Jersey 08854

E-mail address: nussbaum@math.rutgers.edu

Department of Mathematics, Rutgers University, Piscataway, New Jersey 08854

E-mail address: amitpriy@math.rutgers.edu

Mathematisch Instituut, Universiteit Leiden, Leiden, The Netherlands

E-mail address: verduyn@math.leidenuniv.nl 